\title{
SYNERGISTIC EFFECTS IN TWO-PHOTON ABSORPTION: THE QUANTUM ELECTRODYNAMICS OF BIMOLECULAR MEAN-FREQUENCY ABSORPTION
}

\author{
DAVID L. ANDREWS AND KEVIN P. HOPKINS \\ School of Chemical Sciences \\ University of East Anglia \\ Norwich, England
}

\section{CONTENTS}

I. Introduction

II. Quantum Uncertainty and Probability Considerations

A. Energy-Time Uncertainty and Synergistic Ab:orption

1. Cooperative Absorption Mechanism

2. Distributive Absorption Mechanism

B. Probability Considerations

III. Selection Rules

IV. Classification of Synergistic Two-Photon Processes
A. Single-Frequency Excitation
B. Two-Frequency Excitation

V. Quantum Electrodynamics
A. Hamiltonian Representation
B. Time-Dependent Perturbation Theory
C. Tensor Formulation

VI. Rate Equations for Rigidly Oriented Systems
A. Single-Frequency Cooperative Two-Photon Absorption
B. Single-Frequency Distributive Two-Photon Absorption
C. Two-Frequency Cooperative Two-Photon Absorption
D. Two-Frequency Distributive Two-Photon Absorption

VII. Rate Equations for Fluid Media

A. Single-Frequency Cooperative Two-Photon A bsorption

B. Single-Frequency Distributive Two-Photon Absorption

C. Two-Frequency Cooperative Two-Photon Atsorption

D. Two-Frequency Distributive Two-Photon Absorption

VIII. Range-Dependence

IX. Polarization Dependence

A. Single-Beam Polarization Parameters 


\author{
B. Two-Photon Circular Dichroism \\ C. Double-Beam Polarization Studies \\ 1. Natural Two-Photon Circular Dichroism \\ 2. Laser-Induced Circular Dichroism \\ X. Resonance Enhancement
A. Single-Frequency Excitation
B. Two-Frequency Excitation \\ XI. Comparison with Conventional Two-Photon Absorption \\ XII. Synergistic Effects in the Absorption of White Light
A. Spectrophotometry with Broadband Sources
B. Rate Equations for Continuum Excitation
C. Implications for Spectroscopy with USLS Radiation \\ XIII. Chemical Aspects of Synergistic Photoabsorption
A. General Considerations
B. Methods of Observation
C. Charge Transfer Reactions \\ References
}

\title{
I. INTRODUCTION
}

There is a wide range of phenomena in chemical physics resulting from synergistic interactions of atoms and molecules (Schuster et al. 1980). The term synergistic denotes a mechanistic cooperativity, reflected in the fact that complex systems often behave in a very different way from a simple sum of their individual parts. One of the most well-known synergistic effects at the atomic or molecular level is the concerted photoemission process which forms the basis for laser action (Haken 1985). At the other end of the molecular spectrum, synergistic interactions in biomolecules are known to be responsible for many of the control and regulation mechanisms in living organisms (Haken 1977).

In connection with photoabsorption, it has long been known that a collisional interaction between two atoms or molecules irradiated with light of a suitable frequency can result in their simultaneous excitation. The first observations of this effect were made in infrared studies on compressed gases (Ketelaar 1959). Indeed, the effect has been proposed as an explanation for some of the spectral features of planetary atmospheres, where high pressures of gaseous mixtures naturally occur (Danielson 1974). Experimental studies have mostly focused on interaction-induced optical transitions in gases (Yakovlenko 1973; Geltman 1976, 1987; Gallagher and Holstein 1977; Green et al. 1979; Brechignac et al. 1980; Débarre and Cahuzac 1986) or in crystals containing rare earth ions (Varsanyi and Dieke 1961; Dexter 1962; Tulub and Patzer 1968; Nakazawa and Shionoya 1970; Last et al. 1987). Although such processes involve pairs of atoms in collision or close proximity, each absorption involves only a single photon. 
In condensed matter, cooperative effects of a different kind have long been established in connection with the photoproduction of relatively long-lived triplet states, either in individual molecules in the case of liquids (Parker and Hatchard 1962; Nosworthy and Keene 19641, or in triplet excitons in crystalline solids (Avakian and Merrifield 1968; 'Groff et al. 1970). Because of their long lifetimes, the concentrations of such srecies can build up to a point where triplet-triplet annihilation ensues, either jy molecular diffusion or by exciton migration (Craig and Walmsley 1964), resulting in the generation of highly excited states which may subsequertly decay through shortwavelength luminescence (delayed fluorescence) (Siebrand 1965; Smith 1968; Arnold et al. 1970; Sasaki and Hayakawa 1978) These processes often have a quadratic dependence on the intensity of irradiation, and thus exhibit features characteristic of two-photon absorption. However, since the separate photon absorption processes and also the subsequent triplet-triplet annihilation are uncorrelated and individually satisfy the requirements of energy conservation, such phenomena cannot be regarded as truly synergistic in any normal sense of the word.

Where singlet states are concerned, lifetimes are generally too short for concentrations to build up to a point where collisional annihilation is significant, if conventional irradiation sources are employed. With laser light of sufficient intensity, however, such effects are indeed observable and are thought to play a part in the dynamics of energy transfer in photosynthetic systems, for example (Van Grondelle 1985). With laser excitation it is also possible to observe nonlinear optical effects in 'which two or more photons are absorbed by each atomic or molecular pair. The theoretical prediction of such a process was first made by Rios Leite and De Araujo (1980) in a paper concerned with cooperative absorptior by atom pairs in solids. However, the first experimental observation rade shortly afterwards by White (1981) came from laser excitation studies of gaseous mixtures of barium and thallium. Atoms of both species were found to be simultaneously promoted to excited states by a concerted process involving the pairwise absorption of laser photons, a process which thus acquires the character of mean-frequency absorption.

Following theoretical developments (Andrews and Harlow 1983, 1984a), bimolecular mean-frequency absorption was next discovered by researchers working on the photodynamics of charge tranifer reactions. For example, $\mathrm{Ku}$ et al. (1983) performed studies on gaseous mixtures of xenon and chlorine passed through a laser fluorescence cell. The proposed reaction mechanism

$$
\mathrm{Xe}+\mathrm{Cl}_{2}+2 \hbar \omega \rightarrow\left\{\mathrm{Xe}-\mathrm{Cl}_{2}{ }^{* *} \rightarrow \mathrm{Xe}^{+}-\mathrm{Cl}_{2}{ }^{-}\right\} \rightarrow \mathrm{XeCl}+\mathrm{Cl}
$$

was attributed to excitation from the van der Waals ground state potential 
to the ion-pair potential via configurational interaction of the $\mathrm{Xe}$ and $\mathrm{Cl}_{2}$. This discovery clearly delineates the underlying mechanism for the process as one in which the synergistic excitation of the two absorbing species results from molecular proximity rather than any collisional effect. The reaction mechanism (1.1) has been further corroborated by Apkarian and co-workers, who have also extended the study to charge transfer reactions in solid and liquid xenon (Fajardo and Apkarian 1986, 1987, 1988; Wiedeman et al. 1987, 1988). It has additionally been shown to be the predominant reaction route in the case of Xe: $\mathrm{Cl}_{2}$ van der Waals complexes generated in seeded molecular beams (Boivineau et al. 1986a, b).

Recently, a new type of synergistic photoabsorption process involving two-frequency excitation has been the subject of renewed theoretical interest (Andrews and Hopkins 1987, 1988a, 1988b). Here the two chemical centers which undergo concerted excitation may or may not be chemically similar, and can represent either distinct chromophores within a single molecule, loosely bound systems such as van der Waals molecules or solute particles within a coordination shell of solvent molecules, or else completely separate molecules. Where the two centers are chemically identical, the term bicimer (Locke and Lim 1987) appropriately describes the result of the excitation. Two-beam two-photon absorption in lanthanide (III) compounds has been the subject of a recent study by Sztucki and Strek (1988); no experimental studies of synergistic two-beam effects have yet been reported at the time of writing.

While the effects of interest are most readily studied by specifically designed two-beam laser experiments discussed in detail below, it also transpires that the quantum uncertainty mechanism involved may play a significant role in other photoabsorption processes where optical nonlinearity is not immediately apparent. This is particularly the case in connection with studies based on white or broadband light, and the effects may be manifest in the appearance of anomalous features in the corresponding absorption spectra, especially those obtained using ultrashort laser pulses (Andrews 1988). Here, intensity-dependent lineshapes or extinction coefficients and the appearance of ostensibly extraneous spectral lines may all be attributable to the effects of synergistic photoabsorption. It thus appears that synergism in two-photon absorption may have a more general significance than has hitherto been recognized.

\section{QUANTUM UNCERTAINTY AND PROBABILITY CONSIDERATIONS}

Bimolecular mean-frequency absorption is a nonlinear process which results entirely from the effects of molecular proximity, although wavefunction 


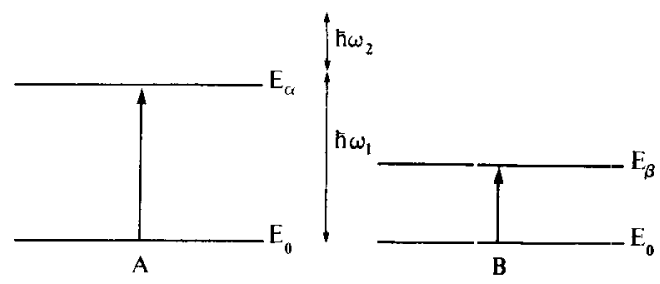

Figure 1. Energy-level diagram for the most genercll case of synergistic two-photon absorption. The absorption of two photons with different frequencies $\omega_{1}$ and $\omega_{2}$ effects transitions to states $|\alpha\rangle$ and $|\beta\rangle$ in two chemically different molecules $A$ and $B$, subject only to overall energy conservation.

overlap is in no way directly implicated. The two chemical centers involved in the process may be either distinct chromoph ores in a single molecule, or completely separate molecules. The two participating centers undergo a concerted excitation through the absorption of two laser photons, and in the most general case the process can be represented by the equation

$$
\mathbf{A}+\mathbf{B}+\hbar \omega_{1}+\hbar \omega_{2} \rightarrow \mathbf{A}^{*}+\mathbf{B}^{*}
$$

which is restricted only by the energy conservation requirement

$$
E_{x 0}+E_{\beta 0}=\hbar \omega_{1}+\hbar \omega_{2}
$$

as illustrated in Fig. 1. It is assumed that both molecules A and B are initially in their ground states, and that they are promoted during the absorption process to excited vibronic states designated by the asterisks in Eq. (2.1), and $\alpha$ and $\beta$ in Eq. (2.2). As will be shown below, this general two-molecule two-photon interaction encompasses four particular types of interaction which are of special interest. However, the basic theory can first be developed with reference to the totally general case.

\section{A. Energy-Time Uncertainty and Syrergistic Absorption}

To understand the physical mechanism underlying these processes, it is helpful to first consider the broad significance of the energy-time Uncertainty Principle for photoabsorption processes. It is well-known that for a molecular excited state with an average lifetime $\delta t$ and an average energy displacement $\delta E$ from the ground state, there exists the relation (Finkel 1987)

$$
\delta t \delta E \geqslant h / 4
$$

The application of this result to the interacion involved in a normal 
photoabsorption process in which individual atoms or molecules absorb single photons amounts to a statement that for a time interval $\delta t$, the mismatch between the energy gain of the absorber and the energy of the absorbed photon cannot exceed $\delta E$. For any normal timescale of observation, this condition ensures that only photons whose energy closely matches a transition energy of the absorber can in fact be absorbed.

The implication of Eq. (2.3) for a concerted photoabsorption process involving the coupling of two atomic or molecular excitations is less obvious, however. In this case, Eq. (2.3) determines the timescale within which the excess energy $\delta E$ absorbed by one center needs to be conveyed to a center with a corresponding negative energy mismatch, in order to fulfil the requirement for long-term energy conservation at each center. There are in fact two distinct mechanisms for a concerted absorption of light based on this Uncertainty Principle, as detailed below.

\section{Cooperative Absorption Mechanism}

Consider first a molecule $\mathrm{A}$ in an initial state $|0\rangle$ undergoing a transition to an excited state $|\alpha\rangle$ through absorption of light with circular frequency $\omega_{1}$. If $\omega_{1}$ is off-resonant with respect to the transition frequency, there is a mismatch in energy by an amount

$$
\delta E=\hbar \omega_{1}-E_{\alpha 0}
$$

Application of the uncertainty relation (2.3) shows that it is impossible to constrain conservation of energy over a timescale less than

$$
\tau=\frac{1}{4}\left(\omega_{1} / 2 \pi-E_{\alpha 0} / h\right)^{-1}
$$

The transition is therefore allowed provided the local energy mismatch exists for a time not exceeding $\tau$.

A suitable mechanism for compensation of the energy mismatch is provided through absorption by a second molecule $B$ of a photon with frequency $\omega_{2}$ given by

$$
\omega_{2}=-\omega_{1}+\left(E_{\alpha 0}+E_{\beta 0}\right) / \hbar
$$

Thus by the cooperative absorption of the photon pair, the total energy absorbed is $\left(E_{\alpha 0}+E_{\beta 0}\right)$, the sum of the two molecular transition energies, and overall energy conservation is therefore achieved.

Clearly there needs to exist a mechanism for conveyance of the mismatch energy from one molecule to another (see Fig. 2), so that over longer times each molecule can individually satisfy the requirements of energy 


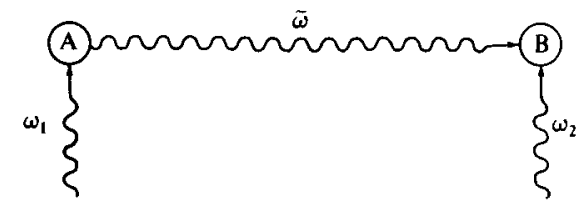

Figure 2. A cooperative mechanism for synergistic two-photon absorption. Molecule A absorbs a photon of frequency $\omega_{1}$ and molecule $B$ a photon of frequency $\omega_{2}$, with the mismatch energy propagated from $A$ to B by a v rtual photon $\tilde{\omega}$.

conservation. Within the framework of quantum electrodynamics (QED), virtual photon coupling (Feynman 1961) provides the mechanism for cooperative absorption of this type. The concept of virtual photons originated in connection with nuclear physics (Dodge 1985), but the formalism has increasingly found application in chemical physics, for example in the theory of intermolecular interactions (Craig and Thirunamachandran 1982; Vigoureux 1983; Grossel et al. 1983; Power and Thirunamachandran 1983; Andrews and Sherborne 1987; Barron and John:ston 1987; Van Labeke et al. 1988) and the calculation of atomic energy shifts (Compagno et al. 1983, 1985).

By introducing the formalism of virtual phciton coupling, the timescale for cooperative absorption, $\tau$, can be interpreted in terms of a range of propagation for which the exchanged photon has virtual character. Thus the distance $R$ between two molecules that cooperate in the absorption process must be subject to the condition

$$
R \leqslant R_{\max } \geqslant \frac{1}{4} h c\left(\hbar \omega_{1}-E_{c: 0}\right)^{-1}
$$

As an example, for a circular frequency mismatich of $2.5 \times 10^{13} \mathrm{~Hz}$, we have the constraint $R_{\max } \geqslant 3 \mu \mathrm{m}$. With a smaller rismatch, $R$ can obviously become very large compared to molecular dimersions, and in the limit where the mismatch is zero, there is no longer any restriction on the molecular separation. This corresponds to the case where two entirely uncorrelated absorption processes occur.

\section{Distributive Absorption M!echanism}

A secondary mechanism for the absorption of nonresonant frequencies involves the concerted absorption of two photons by a single molecule, with virtual photon conveyance of the excess energy to the second absorber (see Fig. 3). As with conventional two-photon absorption, there is no need for

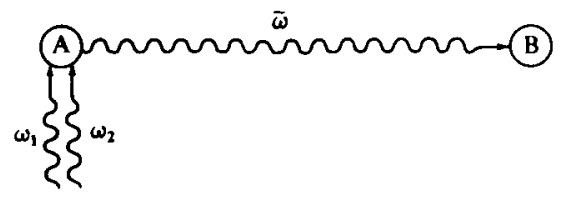

Figure 3. A distributive mechanism for synergistic iwo-photon absorption. Molecule A absorbs two photons of frequencies $\omega_{1}$ and $\omega_{2}$, and the mismatch energy propagates to molecule B by a virtual photon $\tilde{\boldsymbol{\omega}}$. 
the first molecule to possess an energy level corresponding to the energy of either one of the absorbed photons, and thus no identifiable intermediate state is populated, as would be the case in a two-step process. In this case, forthwith termed the distributive mechanism, the two absorbed photons may again have differing energies given by $\hbar \omega_{1}=\left(E_{\alpha 0}+\delta E\right)$ and $\hbar \omega_{2}=\left(E_{\beta 0}-\delta E\right)$, but here the excess energy absorbed by the first molecule now equals the transition energy for the second molecule, $E_{\beta 0}$. The corresponding limit on the range of intermolecular distance is then given by

$$
R \leqslant R_{\max } \geqslant \frac{1}{4} h c / E_{\beta 0}
$$

If the photon energy $\hbar \omega_{1}$ is anywhere near to the excitation energy $E_{\alpha 0}$, it is clear from Eqs. (2.7) and (2.8) that where virtual photon coupling is involved, the distributive mechanism will only be effective over a much shorter range than the cooperative mechanism. For example, if $E_{\alpha 0} / h=5 \times 10^{14} \mathrm{~Hz}$, we have $R_{\max } \geqslant 0.15 \mu \mathrm{m}$.

\section{B. Probability Considerations}

One of the first considerations when comparing the cooperative and distributive mechanisms for synergistic photoabsorption is a difference in the probability aspect of the two processes. At first sight, the requirement of the distributive mechanism for two laser photons to be absorbed in a concerted process at a single molecule appears to render the effect significantly less probable than the cooperative mechanism, which has the apparently looser requirement for two photons to be absorbed by molecules at any two different points in the sample. A simple statistical treatment of each process based on a Poisson distribution, the most appropriate form of distribution for laser light (Louisell 1973), in fact shows that for any given pair of molecules the conditions for the distributive process are met half as often as those of the cooperative process. If the mean number of photons per molecular volume is denoted by $m$, the probability of finding $n$ photons is given by $P_{n}=\left(m^{n} / n !\right) e^{-m}:$ hence for cooperative absorption we have $P_{1}^{2}=m^{2} e^{-2 m}$, and for distributive absorption $P_{2}=m^{2} e^{-2 m} / 2$ (Andrews 1985).

However in most samples of chemical interest there is normally more than one pair of molecules to consider. With $N$ molecules, there are clearly $\frac{1}{2} N(N-1)$ pairs which can participate in a cooperative absorption process, but $N(N-1)$ to participate in a distributive process. Hence, overall the photon statistics do not provide a basis for differentiating the significance of the two mechanisms. However, as shown below, the selection rules for the two processes differ markedly, and often result in a single mechanism being exclusively operative. 


\section{SELECTION RUILS}

As seen above, synergistic two-photon absorption can in principle take place by either or both of the mechanisms, where (i) each laser photon is absorbed by a different molecule (the cooperative mechanism), or (ii) both laser photons are absorbed by a single molecule (the distributive mechanism). In each case, the energy mismatch for the molecular transitiors is transferred between the molecules by means of a virtual photon that couples with each molecule by the same electric-dipole coupling as the laser photons. The result, however, is a significant difference in the selection rules applying to the two types of processes.

In the cooperative case, the two molecular transitions are separately allowed under well-known two-photon selection rules, since each molecule absorbs one laser photon and either emits or a ssorbs a virtual photon. In the same way, the distributive case provides for excitation through three-and one-photon allowed transitions, and may thus lead to excitation of states that are formally two-photon forbidden. (In general, it is sufficient to stipulate that both transitions involved in the distributive mechanism are one-photon allowed since, with the rare exception of icosahedrally symmetric molecules, all transitions which are one-photon allowed are of necessity also three-photon allowed (Andrews and Wilkes 1985).)

Since, on the whole, these processes are of most interest for molecules of fairly high symmetry, it can safely be assumed that in most cases one mechanism alone is involved in the excitation to a particular pair of excited states $\alpha$ and $\beta$. Certainly this is rigorously true for centrosymmetric species, where, under the cooperative mechanism, both transitions must preserve parity ( $g \leftrightarrow \mathrm{g}, \mathrm{u} \leftrightarrow \mathrm{u}$ ), but under the distributive mechanism parity reversal $(\mathrm{u} \leftrightarrow \mathrm{g})$ results at each center. Only in the case of solutions where solute-solvent interactions can reduce excited-state symmetry is this rule weakened (Mohler and Wirth 1988). The assumption that only one mechanism can be operative for any given bimolecular mean-frequency transition gives the advantage of considerably simplifying the form of the rate equations.

One other feature is worth noting at this point, and it concerns the case where the synergistic pair has a fixed mutual crientation, even if the pair itself rotates freely. While the local symmetry of each of the absorbers A and $B$ determines the selection rules for the transitions they undergo, the symmetry of their relative juxtaposition also plays a role in cletermining the polarization characteristics of their synergistic photoabsorption. This is principally manifest in the occurrence of two-photon circular dichroism where the A-B pair has definite handedness, as will be demonst rated in Section IX. Thus it transpires that not only the local symmetry, but also the global symmetry 
of the rotating pair is significant in determining the response to particular polarizations of light.

\section{CLASSIFICATION OF SYNERGISTIC TWO-PHOTON PROCESSES}

As mentioned above, there are four specific cases of bimolecular mean frequency absorption that are of special interest. These are distinguished by the type of mechanism (cooperative or distributive) involved and whether the photons absorbed have the same or different frequencies. The latter condition is in most cases determined by whether a single laser beam or two laser beams are employed for the excitation. We consider first the single-beam cases.

\section{A. Single-Frequency Excitation}

In single-beam bimolecular photoabsorption, the two absorbed photons have the same frequency, and it is the synergistic interaction between two non-identical centers that is of interest. This interaction provides the mechanism for energy exchange such that an overall process

$$
\mathrm{A}+\mathrm{B}+2 \hbar \omega \rightarrow \mathrm{A}^{*}+\mathrm{B}^{*}
$$

can take place even when the individual transitions $\mathrm{A} \rightarrow \mathrm{A}^{*}$ and $\mathrm{B} \rightarrow \mathrm{B}^{*}$ are forbidden on energy grounds, as illustrated in Fig. 4. From a phenomenological viewpoint, the process evidently has the characteristics of meanfrequency photoabsorption. For this effect to be experimentally observable, $\omega$ must be chosen to lie in a region where neither A nor B displays absorption, and we thus have

$$
\hbar \omega=\frac{1}{2}\left(E_{\alpha 0}+E_{\beta 0}\right)
$$

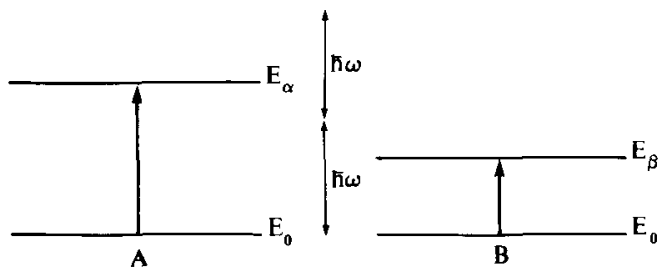

Figure 4. Energy-level diagram for single-beam synergistic absorption. The pairwise absorption of photons with frequency $\omega$ effects transitions to states $|\alpha\rangle$ and $|\beta\rangle$ in two different molecules. 


$$
\hbar \omega \neq E_{\alpha 0}, E_{\beta 0}
$$

So, for two chemically distinct molecules or chromophores A and B, with well-characterized vibronic excited states $\alpha$ and $\beta$, a proximity-induced two-photon absorption process can be induced by tuning the exciting laser to a frequency equivalent to a mean of molecular excitation frequencies for the two molecules.

The two mechanisms for single-beam two-photon absorption are most clearly visualized with the aid of the calculational aids known as time-ordered diagrams. Figure 5(a) shows a typical time-orderıd diagram for a single-beam cooperative two-photon absorption process. Diagrams such as this, originated by Feynman (Feynman 1949; Ward 1965; Wallace 1966), are utilized in QED along with the pertinent equations in order to derive the necessary quantum mechanical probability amplitudes. They can be regarded as a form of space-time diagram with time progressing vertically. The straight lines represent molecules (or atoms) and the wavy lines photons. It is worth emphasizing that such diagrams are purely illustrative devices, representing the ultimately indeterminable sequences of phot on creation and annihilation events involved in a particular process. Thus no importance should be attached to the precise displacements on either the vertical or horizontal axis: it is the ordering of radiation-molecule interactions which each diagram represents that is of importance.

The sequence of events depicted by Fig. 5(al, for example, is as follows: center A first absorbs a real photon of wave vector $\mathbf{k}$ and polarization $\mathbf{e}$, and thereby undergoes a virtual transition to $c . n$ intermediate excited state $|r\rangle$; a virtual photon of wave vector $x$, polarization $\varepsilon$, and frequency $\tilde{\omega}=c|x|$

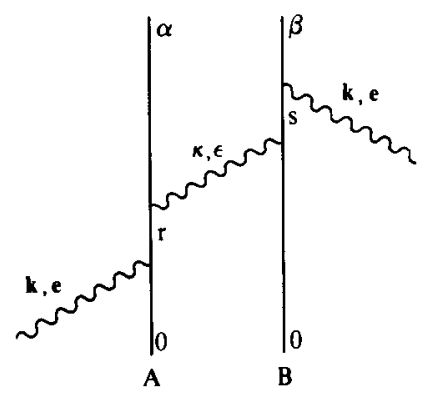

(a)

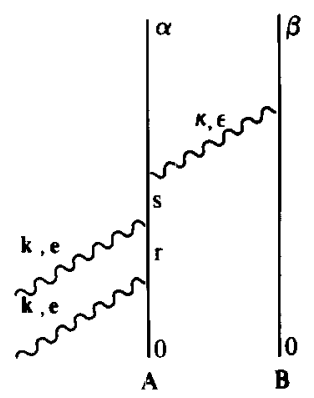

(b)

Figure 5. Typical time-ordered diagrams for single-beain two-photon absorption: (a) shows one of the diagrams associated with the cooperative mech:nism, and (b) one of the diagrams for the distributive mechanism. 
is then created as $A$ adopts its final state $|\alpha\rangle$; the virtual photon propagates from center $A$ to center $B$ where it is annihilated, resulting in the promotion of $\mathbf{B}$ to an excited state $|s\rangle$; finally $\mathrm{B}$ absorbs a second real (laser) photon, and thereby attains its final state $|\beta\rangle$. In total, there are 24 such time-ordered diagrams associated with this process, each corresponding to a topologically different sequence of photon events.

Fig. 5(b) is an illustration of a typical time-ordered diagram for the distributive mechanism. It clearly illustrates the distinguishing feature of this mechanism, in that both laser photons are absorbed by one center; one immediate consequence of this is the above-mentioned difference in the selection rules. Once again, there are 24 such diagrams to be taken into account in the rate calculations. Although development of the theory follows along similar lines for both the cooperative and distributive cases, the dissymmetry of the distributive mode leads to a more complicated rate equation, and when the rate for van der Waals molecules is considered, a new type of rotational average is required. One result of this is, as shown below, the manifestation of two-photon circular dichroism.

\section{B. Two-Frequency Excitation}

In other cases of interest, the two centers have identical chemical composition and are excited by the absorption of two different photons, as, for example, from two different laser beams with frequencies $\omega_{1}$ and $\omega_{2}$. This process can be represented by the equation

$$
\mathrm{A}+\mathrm{A}+\hbar \omega_{1}+\hbar \omega_{2} \rightarrow \mathrm{A}^{*}+\mathrm{A}^{*}
$$

for which the energetics are shown in Fig. 6. Again, for the synergistic process to be observable, the frequencies $\omega_{1}$ and $\omega_{2}$ must be chosen in a region where single-photon absorption cannot lead to the excitation of either center.

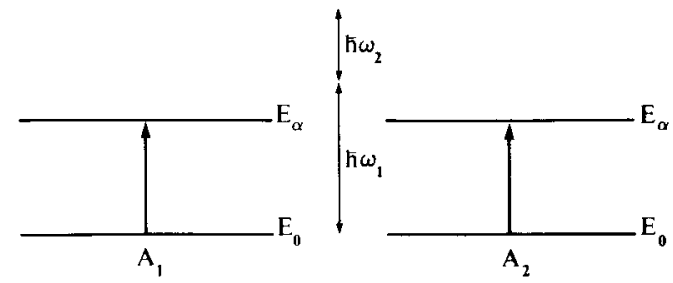

Figure 6. Energy-level diagram for two-beam synergistic absorption. The concerted absorption of one photon from each beam (frequencies $\omega_{1}$ and $\omega_{2}$ ) effects transitions to state $|\alpha\rangle$ in two chemically identical molecules. 
Thus we have

$$
\begin{array}{r}
\frac{1}{2}\left(\hbar \omega_{1}+\hbar \omega_{2}\right)=E_{\alpha 0} \\
\hbar \omega_{1}, \hbar \omega_{2} \neq E_{\alpha 0}
\end{array}
$$

The former relation, Eq. (4.5), indicates the fact that this cooperative process again has the characteristics of mean-frequency absorption: here, however, it is the molecular excitation frequency which equals the mean of the two photon frequencies.

From an experimental point of view, the do greater flexibility, in that some choice can be exercized in the individual frequencies of the two laser beams, subject to the satisfaction of Eq. (4.5). It is therefore normally possible to avoid tuning either laser to a frequency that might swamp the bimolecular process with conventional single-photon absorption. The utilization of resonances with in termediate energy levels (see Section $\mathrm{X}$ ) is also facilitated by the ability to tune one of the lasers while keeping the mean value of the two laser frequericies fixed.

The absorption of two different photons alsis significantly increases the number of polarization variables arising in the rate equations. The ability of the experimentalist to independently vasry the polarization and experimental configuration of the two laser bearns allows a choice of values for these polarization parameters which significantly increases the amount of information that can be derived from the spestra. In contrast to the case of single-beam excitation, this also affords the opportunity to observe the induction of circular dichroism in a system of achiral molecules.

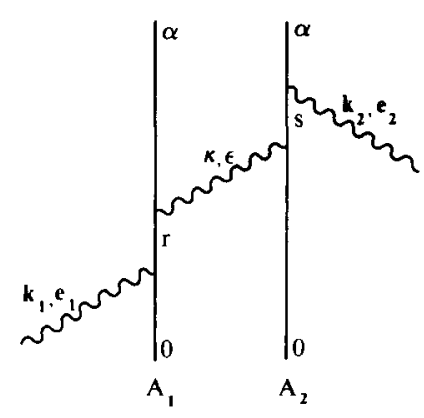

(a)

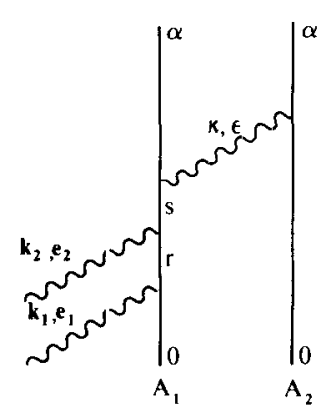

(b)

Figure 7. Typical time-ordered diagrams for two-beam two-photon absorption: (a) shows one of the diagrams associated with the cooperative mechanism, and (b) one of the diagrams for the distributive mechanism. 
A typical time-ordered diagram for the cooperative two-beam process is illustrated in Fig. 7(a). Although it bears a close resemblance to Fig. 5(a), the appearance of two laser photons with differing frequencies $\left(\omega_{1}\right.$ and $\left.\omega_{2}\right)$ produces a total of 48 time-ordered diagrams, as opposed to the 24 that occur in the single-beam case. Another 48 diagrams are required for representation of the corresponding distributive case, as typified by Fig. 7(b), where the virtual photon carries the entire excitation energy from one molecule to the other. The comments made in the preceding section regarding the single- and double-beam cooperative mechanisms again apply here, and once more the corresponding difference in the selection rules associated with the distributive mechanism ensues.

In passing, we note that parametric four-wave mixing processes could in principle contribute to the effects described above. Thus, in the single-beam case, the CARS process $\omega+\omega \rightarrow \omega_{1}+\omega_{2}$ at a single center could also result in the synergistic excitation of two chemically different centers. Equally in the double-beam case, the four-wave interaction $\omega_{1}+\omega_{2} \rightarrow \omega+\omega$ followed by absorption of the frequency $\omega$, could contribute to the excitation of a pair of neighboring molecules of the same species. However, both of these four-wave interactions will be relatively ineffectual unless (one of) the emission frequencies is stimulated by an additional source; moreover, the processes described here are not associated with the wave-vector matching characteristics of CARS and related phenomena.

\section{QUANTUM ELECTRODYNAMICS}

The theory of synergistic effects in two-photon absorption is based upon the standard methods of molecular quantum electrodynamics (QED). The fundamental development of these methods is delineated in the book by Craig and Thirunamachandran (1984), which has established the rigorous use of SI units in molecular QED. The same conventions are adopted in this review, representing a departure from two early papers (Andrews and Harlow 1983, 1984a) in which equations were cast in Gaussian units.

To develop the basic formalism, we initially consider the most general case as represented by Eq. (2.1), i.e., the process in which two molecules labelled $\mathrm{A}$ and $\mathrm{B}$ are excited to final states $|\alpha\rangle$ and $|\beta\rangle$, respectively, through the absorption of two different laser photons. It is assumed that these are derived from two separate beams simultaneously traversing the sample. The first beam is characterized by wave vector $k_{1}$, polarization $\mathbf{e}_{1}$ and frequency $\omega_{1}\left(=c\left|\mathbf{k}_{1}\right|\right)$, while beam 2 is similarly characterized by $\mathbf{k}_{2}, \mathbf{e}_{2}$ and $\omega_{2}$, and the absorption process satisfies the energy conservation relation

$$
\hbar \omega_{1}+\hbar \omega_{2}=E_{\alpha 0}+E_{\beta 0} .
$$


The specific cases where: (i) A and B are identical molecules, or (ii) the two absorbed photons are identical are considered in detail subsequently.

\section{A. Hamiltonian Representation}

We begin by writing down the quantum electrodynamical Hamiltonian for the system comprising the radiation and the two molecules $\mathrm{A}$ and $\mathrm{B}$. We adopt the Hamiltonian given by the Power-Zienalu-Woolley transformation, which may be expressed as follows (Power and Zisnau 1959; Woolley 1971)

$$
H=H_{\mathrm{rad}}+\sum_{\zeta=\mathrm{A}, \boldsymbol{B}} H_{\mathrm{mol}}(\zeta)+\sum_{\zeta=\mathbf{A}, B} H_{\mathrm{int}}(\zeta)
$$

Here $H_{\text {rad }}$ is the radiation field Hamiltonian given by

$$
H_{\mathrm{rad}}=\left(\varepsilon_{0} / 2\right) \int\left\{\varepsilon_{0}^{-2} \mathbf{d}^{\perp 2}(\mathbf{r})+c^{2} \mathbf{b}^{2}(\mathbf{r})\right\} d^{3} \mathbf{r}
$$

where $\mathbf{d}^{\perp}$ and $\mathbf{b}$ are the transverse electric field and magnetic field operators, respectively; $H_{\text {mol }}(\zeta)$ is the nonrelativistic Schrödinger operator for the molecule $\zeta$; and $H_{\text {int }}(\zeta)$ is the Hamiltonian representing the molecular interaction with the radiation.

It is important to note that there is no intermolecular Coulomb potential in Eq. (5.2); in the Power-Zienau-Woolley formalism all intermolecular interactions are mediated by a coupling to the radiation field. It is for this reason that any process involving the transfer o: energy from one molecule to another must be calculated on the basis of an exchange of virtual photons between the two molecules. The quantum electrodynamical method automatically incorporates the same kind of retardation effects as those which, for example, modify the $R^{-6}$ dependence of the exchange interaction to $R^{-7}$ at large distances (Casimir and Polder 1948). S milar variations in inverse power with distance have recently been noted ir connection with electronatom scattering (Au 1988).

The explicit form of the interaction Hamiltonian $H_{\text {int }}(\zeta)$ consists of a series of multipolar terms, but for most purposes the electric-dipole (E1) approximation is sufficient. Although the results are calculated within this approximation for each molecular center $\zeta$, detailed analysis of the coupling provides results equivalent to the inclusion of higher-order multipole terms for the pair. The same assumption underlies the well-known coupledchromophore model of optical rotation (Kuhn 1930; Boys 1934; Kirkwood 1937). The Hamiltonian for the system may thus be written as

$$
H_{\mathrm{int}}(\zeta)=-\varepsilon_{0}^{-1} \boldsymbol{\mu}(\zeta) \cdot \mathbf{d}^{\perp}\left(\mathbf{R}_{\zeta}\right)
$$


where $\boldsymbol{\mu}(\zeta)$ is the electric-dipole moment operator for molecule $\zeta$ located at position $\mathbf{R}_{\zeta}$. The transverse electric displacement operator for the radiation field, $\mathbf{d}^{+}$, can be written as a summation over radiation modes:

$$
\mathbf{d}^{\perp}(\mathbf{r})=\mathrm{i} \sum_{\mathbf{k}, \lambda}\left\{\hbar c k \varepsilon_{0} / 2 V\right\}^{1 / 2}\left\{\mathbf{e}^{(\lambda)}(\mathbf{k}) a^{(\lambda)}(\mathbf{k}) e^{i \mathbf{k} \cdot \mathbf{r}}-\overline{\mathbf{e}}^{(\lambda)}(\mathbf{k}) a^{+(\lambda)}(\mathbf{k}) e^{-i \mathbf{k} \cdot \mathbf{r}}\right\}
$$

Here $\mathbf{e}^{(\lambda)}(\mathbf{k})$ is the unit polarization vector for the mode characterized by propagation vector $\mathbf{k}$ and polarization $\lambda$, with a frequency given by $\omega=\mathbf{c}|\mathbf{k}|$; $a^{+(\lambda)}(\mathbf{k})$ and $a^{(\lambda)}(\mathbf{k})$ are, respectively, the corresponding creation and annihilation operators, and $V$ is the quantization volume.

\section{B. Time-Dependent Perturbation Theory}

The basis for the rate calculations is time-dependent perturbation theory. This is utilized to calculate an absorption rate $\Gamma$ from the Fermi Golden Rule (Schiff 1968):

$$
\Gamma=(2 \pi / \hbar)\left|M_{f i}\right|^{2} \rho_{f}
$$

where the transition matrix element (probability amplitude) is given by

$$
\begin{aligned}
M_{f i}= & \left\langle f\left|H_{\mathrm{int}}\right| i\right\rangle+\sum_{r} \frac{\left\langle f\left|H_{\mathrm{int}}\right| r\right\rangle\left\langle r\left|H_{\mathrm{int}}\right| i\right\rangle}{E_{i}-E_{r}} \\
& +\sum_{s, r} \frac{\left\langle f\left|H_{\mathrm{int}}\right| s\right\rangle\left\langle s\left|H_{\mathrm{int}}\right| r\right\rangle\left\langle r\left|H_{\mathrm{int}}\right| i\right\rangle}{\left(E_{i}-E_{s}\right)\left(E_{i}-E_{r}\right)} \\
& +\sum_{t, s, r} \frac{\left\langle f\left|H_{\mathrm{int}}\right| t\right\rangle\left\langle t\left|H_{\mathrm{int}}\right| s\right\rangle\left\langle s\left|H_{\mathrm{int}}\right| r\right\rangle\left\langle r\left|H_{\mathrm{int}}\right| i\right\rangle}{\left(E_{i}-E_{\mathrm{t}}\right)\left(E_{i}-E_{s}\right)\left(E_{i}-E_{r}\right)}+\cdots
\end{aligned}
$$

All states appearing in this expression are states of the system that comprises the radiation and the two molecules $\mathrm{A}$ and $\mathrm{B}$. The symbols $r, s$, and $t$ denote intermediate states, while $i$ and $f$ represent the initial and final states. The summations are performed over all intermediate states of the system, i.e., all states excluding the initial and final states. These are represented by

$$
\begin{aligned}
& |i\rangle=\left|0 ; 0 ; n_{1} ; n_{2} ; 0\right\rangle \\
& |f\rangle=\left|\alpha ; \beta ;\left(n_{1}-1\right) ;\left(n_{2}-1\right) ; 0\right\rangle
\end{aligned}
$$

where the sequence in the ket denotes: | the state of $\mathrm{A}$; the state of $\mathrm{B}$; the number of photons in beam 1; the number of photons in beam 2; the number of virtual photons $>$. 
From Eqs. (5.4) and (5.5) it follows that sach appearance of $H_{\text {int }}$ is associated with the creation or annihilation of a photon. It is thus readily apparent that the first non-zero contribution from Eq. (5.7) is the fourth-order term, corresponding to four separate photon creation and annihilation events; these comprise the two annihilations of real photons from the incident light, and the creation and annihilation of the virtual photon which couples the two molecules.

The complete set of interaction sequences incorporated in the fourth-order term for $M_{f i}$ are accounted for by 96 time-ordesed diagrams, in the general case, 48 of which are associated with the cooperative mechanism and 48 with the distributive mechanism: examples of each type are shown in Fig. 8. The matrix element contribution for the cooperative mechanism corresponding to Fig. 8(a), for example, when evaluated using Eq. (5.7) gives the following result;

$$
\begin{aligned}
M_{f i}^{(1)}= & \sum_{r} \sum_{s} \sum_{\chi} \sum_{\varepsilon}\left\langle\alpha ; \beta ; n_{1}-1 ; n_{2}-1 ; 0\left|H_{\mathrm{int}}\right| \alpha ; s ; n_{1}-1 ; n_{2} ; 0\right\rangle \\
& \times\left\langle\alpha ; s ; n_{1}-1 ; n_{2} ; 0\left|H_{\mathrm{int}}\right| \alpha ; 0 ; n_{1}-1 ; n_{2} ; 1\right\rangle \\
& \times\left\langle\alpha ; 0 ; n_{1}-1 ; n_{2} ; 1\left|H_{\mathrm{int}}\right| r ; 0 ; n_{1}-1 ; n_{2} ; 0\right\rangle \\
& \times\left\langle r ; 0 ; n_{1}-1 ; n_{2} ; 0\left|H_{\mathrm{int}}\right| 0 ; 0 ; n_{1} ; n_{2} ; 0\right\rangle \\
& \times\left\{\left(E_{0 \alpha}+E_{0 s}+\hbar \omega_{1}\right)\left(E_{0 \alpha}+\hbar \omega_{1}-h c \chi\right)\left(E_{0 r}+\hbar \omega_{1}\right)\right\}^{-1},
\end{aligned}
$$

It is important to re-emphasize that no single time-ordered diagram represents a physically distinguishable process: the diagrams are ultimately only calculational aids based on the approximations of perturbation theory.

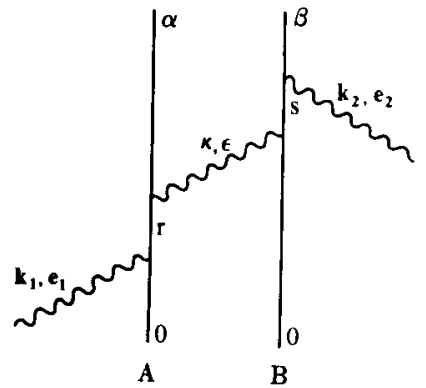

(a)

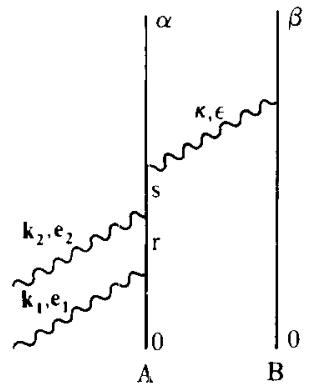

(b)

Figure 8. Typical time-ordered diagrams for the most general case of synergistic absorption with two-beam excitation of a chemically dissimilar pair of molecules: (a) relates to the cooperative mechanism and (b) the distributive mechanism. 
In fact, the photon creation and annihilation events at each molecule appear simultaneous, as far as real experimental measurements with finite time resolution are concerned. However, the time-energy uncertainty relation does permit short-lived states that are not properly energy-conserving. This helps explain why it is necessary to include diagrams corresponding to time sequences in which a virtual photon is created before either real photon arrives. It nonetheless transpires that such apparently unphysical cases produce the smallest contributions to the matrix element.

Working in a similar way, it is found that there are 24 contributions arising from diagrams of the distributive type represented in Fig. 8(b), where both real photons are absorbed at center $\mathrm{A}$, and a virtual photon conveys the energy mismatch to $\mathrm{B}$; the final 24 distributive contributions arises from the mirror-image case where both real photons are absorbed at $B$ and the virtual photon propagates to A. The addition of all 96 matrix element contributions then produces the complete fourth-order result for $M_{f i}$.

Once the summation over virtual photon wave-vectors and polarizations in Eq. (5.10) is performed, the result can be cast in terms of a retarded resonance electric dipole-electric dipole interaction tensor $V_{k l}(\omega, \mathbf{R})$ (Power and Thirunamachandran 1983; Andrews and Sherborne 1987), using the identity

$$
\left(\frac{\hbar c}{2 V \varepsilon_{0}}\right) \sum_{\chi, \varepsilon} \varkappa \varepsilon_{k} \bar{\varepsilon}_{l}\left[\frac{e^{i \chi, \mathbf{R}}}{(\hbar \omega-\hbar c \varkappa)}-\frac{e^{-i \chi, \mathbf{R}}}{(\hbar \omega+\hbar c \varkappa)}\right]=V_{k l}(\omega, \mathbf{R})
$$

where $\mathbf{R}$ denotes the vector displacement $\mathbf{R}(B)-\mathbf{R}(A)$, and $V_{k l}(\omega, \mathbf{R})$ is given by;

$$
\begin{aligned}
V_{k l}(\omega, \mathbf{R})= & \left(1 / 4 \pi \varepsilon_{0} R^{3}\right)\left[\left\{\left(\delta_{k l}-3 \hat{\mathbf{R}}_{k} \hat{\mathbf{R}}_{l}\right)\left(e^{i \omega R / c}-(i \omega R / c) e^{i \omega R / c}\right)\right.\right. \\
& \left.\left.-\left(\delta_{k l}-\hat{\mathbf{R}}_{k} \hat{\mathbf{R}}_{l}\right)(\omega R / c)^{2} e^{i \omega R / c}\right\}\right]
\end{aligned}
$$

which has the property $\bar{V}_{k l}(\omega, R)=V_{k l}(-\omega, R)$. The leading term of Eq. (5.12) in square brackets, with $R^{-3}$ dependence, designates a static interaction, the following term with $R^{-2}$ dependence an inductive interaction, and the last term a radiative interaction. The effect of incorporating higher-order multipolar contributions to the interaction tensor has recently been discussed by Thirunamachandran (1988), and Vigoureux et al. (1987) have shown how to adapt the formalism to include coupling with surface modes in the case of adsorbate molecules.

\section{Tensor Formulation}

The matrix element for the absorption process may now be expressed in terms of molecular tensors $S^{\alpha 0}, S^{\beta 0}, \chi^{\alpha 0}, \chi^{\beta 0}$, as follows, using the convention 
of implied summation over repeated tensor indices:

$$
\begin{aligned}
M_{f i}= & K\left\{e_{1 i} e_{2 j} S_{i k}^{\alpha 0}\left(\omega_{1}\right) S_{j l}^{\beta 0}\left(\omega_{2}\right) V_{k l}\left(\left[\omega_{\beta 0}-\omega_{2}\right], \mathbf{R}\right) \exp \left(i\left[\mathbf{k}_{1} \cdot \mathbf{R}_{A}+\mathbf{k}_{2} \cdot \mathbf{R}_{B}\right]\right)\right. \\
& +e_{1 i} e_{2 j} S_{i k}^{\beta 0}\left(\omega_{1}\right) S_{j l}^{\alpha 0}\left(\omega_{2}\right) V_{k l}\left(\left[\omega_{\beta 0}-\omega_{1}\right], \mathbf{R}\right) \exp \left(i\left[\mathbf{k}_{2} \cdot \mathbf{R}_{A}+\mathbf{k}_{1} \cdot \mathbf{R}_{B}\right]\right) \\
& \left.+e_{1 i} e_{2 j} \chi_{i j k}^{\alpha 0}\left(A, \omega_{1}, \omega_{2}\right) \mu_{l}^{\beta 0} V_{k l}\left(\omega_{\beta 0}, \mathbf{R}\right) \operatorname{ex}\right]\left(i\left[\mathbf{k}_{1}+\mathbf{k}_{2}\right] \cdot \mathbf{R}_{A}\right) \\
& \left.+e_{1 i} e_{2 j} \chi_{i j k}^{\beta 0}\left(B, \omega_{1}, \omega_{2}\right) \mu_{l}^{\alpha 0} \bar{V}_{k l}\left(\omega_{\alpha 0}, \mathbf{R}\right) \operatorname{exf}\left(i\left[\mathbf{k}_{1}+\mathbf{k}_{2}\right] \cdot \mathbf{R}_{B}\right)\right\}
\end{aligned}
$$

where

$$
K=-\left(\hbar c / 2 V \varepsilon_{0}\right)\left(n_{1} n_{2} k_{1} k_{2}\right)^{1 / 2}
$$

(A slightly different formula applies when the frequencies $\omega_{1}$ and $\omega_{2}$ become equal, as will be seen in the next section.) The parameters $\omega_{\alpha 0}$ and $\omega_{\beta 0}$ in Eq. (5.13) are defined by $\hbar \omega_{\alpha 0}=E_{\alpha 0}$ and $\hbar \omega_{\beta 0}=E_{\beta 0}$, and effectively represent the conservation energy transferred between the two centers by the virtual photon.

The first two terms in Eq. (5.13) arise from the cooperative mechanism, while the distributive mechanism gives rise to the third and fourth terms. Deriving the general rate for a proximity-induced two-photon absorption process from the square modulus of the result is an ellaborate procedure producing sixteen terms, including cross-terms associated with quantum mechanical interference between the cooperat ve and distributive mechanisms. However, in view of the selection rule: discussed earlier, it is not generally necessary to perform this calculation since each of the four specific mechanisms for two-photon absorption under consideration can, at most, have only two terms of Eq. (5.13) contributing to the matrix element.

At this stage, it is appropriate to describe the detailed structure and properties of the molecular tensors appearing in Eq. (5.13). The explicit form of the second-rank molecular response tensor $\mathbf{S}_{i j}^{f 0}(\omega)$ is

$$
\mathbf{S}_{i j}^{f o}(\omega)=\sum_{r}\left[\frac{\mu_{i}^{f r} \mu_{j}^{r o}}{\left(E_{f r}-\hbar \omega\right)}-\frac{u_{j}^{f r} \mu_{i}^{r o}}{\left(E_{r o}-\hbar \omega\right)}\right]
$$

It is readily shown that this is identically equal to the electronic Raman scattering tensor for the Raman transition $|f\rangle \leftarrow|0\rangle$. Note that the first term in Eq. (5.15) dominates if there exists a state $r>$ such that $E_{f r} \approx \hbar \omega$; the second term dominates if there is a state such that $E_{r 0} \approx \hbar \omega$. Such cases lead to resonance enhancement and will be discussed in a more detailed manner later. The molecular tensors $\mathbf{S}_{i k}^{\beta 0}\left(\omega_{1}\right), \mathbf{S}_{j l}^{\alpha 0}\left(\omega_{2}\right)$, stc., are to be understood as generalizations of the above equation. 
The third-rank molecular tensor $\chi_{i j k}^{f 0}$ is defined as

$$
\begin{aligned}
\chi_{i j k}^{f o}\left(\omega_{1}, \omega_{2}\right)= & \sum_{s, r}\left[\frac{\mu_{i}^{r o} \mu_{j}^{s r} \mu_{k}^{f s}}{\left(E_{o s}+\hbar \omega_{1}+\hbar \omega_{2}\right)\left(E_{o r}+\hbar \omega_{1}\right)}+\frac{\mu_{i}^{r o} \mu_{j}^{f s} \mu_{k}^{s r}}{\left(E_{f s}-\hbar \omega_{2}\right)\left(E_{o r}+\hbar \omega_{1}\right)}\right. \\
& +\frac{\mu_{i}^{s r} \mu_{j}^{f s} \mu_{k}^{r o}}{\left(E_{f r}-\hbar \omega_{1}-\hbar \omega_{2}\right)\left(E_{f s}-\hbar \omega_{2}\right)}+\frac{\mu_{i}^{s r} \mu_{j}^{r o} \mu_{k}^{f s}}{\left(E_{o s}+\hbar \omega_{1}+\hbar \omega_{2}\right)\left(E_{o r}+\hbar \omega_{2}\right)} \\
& \left.+\frac{\mu_{i}^{f s} \mu_{j}^{r o} \mu_{k}^{s r}}{\left(E_{o r}+\hbar \omega_{2}\right)\left(E_{f s}-\hbar \omega_{1}\right)}+\frac{\mu_{i}^{f s} \mu_{j}^{s r} \mu_{k}^{r o}}{\left(E_{f r}-\hbar \omega_{1}-\hbar \omega_{2}\right)\left(E_{f s}-\hbar \omega_{1}\right)}\right]
\end{aligned}
$$

This molecular response tensor is a more general form of three apparently different tensors which have featured in previous work on multiphoton processes; one is the $\chi_{i j k}^{f 0}$ tensor arising in the single-frequency distributive two-photon absorption (Andrews and Harlow 1984a), and another is the $\mathbf{T}_{i j k}$ tensor which appears in the theory of three-photon absorption (Andrews and Wilkes 1985). It is also exactly identical to the two-frequency hyperRaman transition tensor $\boldsymbol{\beta}_{i j k}^{f 0}$ (Andrews 1984).

In subsequent development of the synergistic rate equations, the parametric dependence on frequency of $\mathbf{S}_{i j}^{f 0}$ and $\chi_{i j k}^{f 0}$ is left implicit so as to avoid unnecessary congestion, and $\omega_{1}=\omega_{2}=\omega$ for single-beam cases. It is not generally necessary to label tensors $\mathrm{A}$ or $\mathrm{B}$ to denote at which center they are to be evaluated, since the superscript $\alpha$ or $\beta$ designates whether each tensor represents interactions at center A or B. In the double-beam case it proves necessary for calculational purposes to distinguish tensors associated with the two (chemically identical) centers by labeling them $A_{1}$ and $A_{2}$.

\section{RATE EQUATIONS FOR RIGIDLY ORIENTED SYSTEMS}

The rate of a general, proximity-induced, two-photon absorption process can be calculated by combining the Fermi Golden rule, Eq. (5.6), with Eq. (5.13). For each of the four specific cases to be studied, the selection rules normally dictate that only two of the four terms in Eq. (5.13) arise for any given mechanism, thus reducing the number of terms in the rate equation to just four. A brief derivation of the rate of absorption for each case is presented below.

\section{A. Single-Frequency Cooperative Two-Photon Absorption}

The single-beam cooperative mechanism applies where a single laser beam is utilized to excite the sample, and where transitions in both species A and 
$\mathrm{B}$ are forbidden as single-photon processes, but allowed under two-photon selection rules. This mechanism is represented by the time-ordered diagram of Fig. 5(a), and is governed by the energy conservation relations of Eqs (4.2) and (4.3).

The relevant transition matrix element can $x$ obtained by examination of Eq. (5.13). Setting $\omega_{1}=\omega_{2} \equiv \omega$, and dropping forbidden contributions from the third and fourth terms, the matrix ele ment becomes:

$$
M_{f i}=K^{\prime} e_{i} e_{j} S_{i k}^{\alpha 0}(\omega) S_{j l}^{\beta 0}(\omega) V_{k l}\left(\left[\omega_{\beta 0}-\omega\right], \mathbf{R}\right) \exp \left(i \mathbf{k} \cdot\left[\mathbf{R}_{A}+\mathbf{R}_{B}\right]\right)
$$

where $K^{\prime}$ is given by

$$
K^{\prime}=-\left(\hbar c k / 2 V \varepsilon_{0}\right) n^{1 / 2}(n--1)^{1 / 2}
$$

This constant is essentially the limit of $K$ as given by Eq. (5.14), in the case where the two absorbed photons become identical; however, the factor $\left(n_{1} n_{2}\right)^{1 / 2}$ is replaced by $n^{1 / 2}(n-1)^{1 / 2}$ since the photon annihilation operator acts twice on the same radiation mode. As will be seen below, this difference is ultimately reflected in a dependence on the coherence properties of the laser source, which is uniquely associated with single-beam processes. It is also worth observing that although the first two terms of Eq. (5.13) become identical if the two absorbed photons are derived from the same beam, inclusion of a factor of 2 in Eq. (6.1) would amount to double-counting the time-ordered diagrams, and is therefore not appropriate.

The rate for the process may now be calculated from the Fermi rule, and is given by;

$$
\begin{aligned}
\Gamma_{1 c}= & \left(2 \pi \rho_{f} / \hbar\right) K^{\prime 2}\left\{e_{i} e_{j} \bar{e}_{m} \bar{e}_{n} S_{i k}^{\alpha 0}(\omega) S_{j l}^{\beta C}(\omega) \bar{S}_{m o}^{\alpha 0}(\omega) \bar{S}_{n p}^{\beta 0}(\omega)\right. \\
& \left.\times V_{k l}\left(\left[\omega_{\beta 0}-\omega\right], \mathbf{R}\right) \bar{V}_{o p}\left(\left[\omega_{\beta 0}-\omega\right], \mathbf{R}\right)\right\}
\end{aligned}
$$

This S.I. result corresponds to the expression originally derived in Gaussian units by Andrews and Harlow (1983). As it stand:;, all molecular and radiative parameters in the above expression are referred to a single Cartesian frame of reference, making the result directly applicable only to rigidly oriented molecules or solids. For application to fluid sa nples, further processing of the result to account for molecular orientation is necessary before this result and others below can be applied; the procedı.re is discussed in detail in Section VII.

To complete the calculation, certain photon statistical features of the result must be examined and recast in terms of experimentally determinable parameters. The problem lies in the constant $K^{\prime}$ which, defined through 
Eq. (6.2) in terms of photon number $n$ and quantization volume $V$, is not directly amenable to experimental application. Moreover, since the quantization volume is merely a theoretical artefact, it must invariably cancel out in any final rate equation. However the ratio of photon number and quantization volume is directly related to the mean irradiance $I$; the relationship is as follows;

$$
I=n \hbar c^{2} k / V
$$

A secondary problem lies in the fact that the number states employed in Section $\mathrm{V}$, which formally represent radiation for which there is a precise nonfluctuating value for the number of photons, provide only a poor representation of coherent laser light. More physical results can be obtained by considering the photon number to be subject to fluctuations which satisfy particular types of statistical distribution. By suitably weighting rate equations calculated on the basis of number states, various kinds of radiation can then be modeled. The result is a replacement of the factor $n(n-1)$ by $g_{12}^{(2)} \bar{n}^{2}$, where $\bar{n}$ is the mean photon number and $g_{12}^{(2)}$ is the degree of second-order coherence of the beam (Loudon 1983). This quantity depends on the detailed statistical properties of the light source, provided the two absorptions which it correlates occur within the coherence length of the radiation. For coherent light $g_{12}^{(2)}$ takes the value of unity; for thermal light it equals 2 , and other values typify different kinds of photon distribution. Taking these factors into account, it transpires that $K^{\prime 2}$ is more properly expressed in terms of the mean irradiance $\bar{I}$ and the degree of second-order coherence by the quite general result:

$$
K^{\prime 2}=\left(1 / 4 c^{2} \varepsilon_{0}^{2}\right) \bar{I}^{2} g_{12}^{(2)}
$$

\section{B. Single-Frequency Distributive Two-Photon Absorption}

In this mechanism, two-photon transitions are forbidden and the excitation of the participating molecules occurs through one- and three-photon allowed transitions. Both the real (laser) photons are absorbed by one molecule, excitation of its partner resulting from the virtual photon coupling. Because of the difference in selection rules from the previous case, the first two terms of Eq. (5.13) are now zero, and contributions arise only from the third and fourth terms. It must also be noted that setting the two absorbed photon frequencies to be equal in Eq. (5.16) to produce $\chi_{(i j) k}^{\alpha 0}(\omega, \omega)$ introduces index symmetry into the tensor, as indicated by the brackets embracing the first two indices. A factor of $\frac{1}{2}$ must then be introduced into the definition of this tensor in order to avoid over-counting contributions. The transition matrix 
element for this mechanism is then as follows:

$$
\begin{aligned}
M_{f i}= & K^{\prime} e_{i} e_{j}\left\{\chi_{(i j) k}^{\alpha 0} \mu_{l}^{\beta 0} V_{k l}\left(\omega_{\beta 0}, \mathbf{R}\right) \exp \left(2 i \mathbf{k} \cdot \mathbf{R}_{A}\right)\right. \\
& \left.+\chi_{(i j) k}^{\beta 0} \mu_{l}^{\alpha 0} \bar{V}_{k l}\left(\omega_{\alpha 0}, \mathbf{R}\right) \exp \left(2 i \mathbf{k} \cdot \mathbf{R}_{B}\right)\right\}
\end{aligned}
$$

Taking the square modulus of this result to evaluate the overall rate from Eq. (5.6) again introduces a factor of $K^{\prime 2}$, which can be evaluated from Eq. (6.5) subject to replacement of $g_{12}^{(2)}$ by $g_{11}^{(2)}$, (which is independent of coherence length), the subscripts in the latter case denoting the fact that both laser photons are absorbed by the same molecule. The final result for the rate is then given by;

$$
\begin{aligned}
\Gamma_{1 d}= & \left(2 \pi \rho_{f} / \hbar\right) K^{\prime 2} e_{i} e_{j} \bar{e}_{m} \bar{e}_{n}\left\{\chi_{(i j) k}^{\alpha 0} \mu_{l}^{\beta 0} \bar{\chi}_{(m n) o}^{\alpha 0} \bar{\mu}_{p}^{\beta 0} V_{k l}\left(\omega_{\beta 0}, \mathbf{R}\right) \bar{V}_{o p}\left(\omega_{\beta 0}, \mathbf{R}\right)\right. \\
& +\chi_{(i j) k}^{\beta 0} \mu_{l}^{\alpha 0} \bar{\chi}_{(m n) o}^{\beta 0} \bar{\mu}_{p}^{\alpha 0} \bar{V}_{k l}\left(\omega_{\alpha 0}, \mathbf{R}\right) V_{o p}\left(\omega_{\alpha 0}, \mathbf{R}\right) \\
& +\chi_{(i j) k}^{\beta 0} \mu_{l}^{\alpha 0} \bar{\chi}_{(m n) o}^{\alpha 0} \bar{\mu}_{p}^{\beta 0} \bar{V}_{k l}\left(\omega_{\alpha 0}, \mathbf{R}\right) \bar{V}_{o p}\left(\omega_{\beta c}, \mathbf{R}\right) \exp (2 i \mathbf{k} \cdot \mathbf{R}) \\
& \left.+\chi_{(i j) k}^{\alpha 0} \mu_{l}^{\beta 0} \bar{\chi}_{(m n) o}^{\beta 0} \bar{\mu}_{p}^{\alpha 0} V_{k l}\left(\omega_{\beta 0}, \mathbf{R}\right) V_{o p}\left(\omega_{\alpha c}, \mathbf{R}\right) \exp (-2 i \mathbf{k} \cdot \mathbf{R})\right\}
\end{aligned}
$$

The tensor indices in Eq. (6.7) are now referred to a molecular frame of reference arbitrarily centered on molecule $A$, thereby reducing the value of $R_{A}$ to zero. The result is again equivalent to a result given previously in Gaussian units (Andrews and Harlow 1984a), although it is important to note that only the real part of the retarded resonance electric dipole-electric dipole interaction tensor, $V_{k l}$, was employed in the original derivation. Subsequent studies have shown that it is in fast necessary to employ this interaction tensor in its complete, complex form, rather than simply utilizing the real part (Power and Thirunamachandran 1983). The persistence of phase factors in the rate equation should be noted, and the physical significance of this will become evident in a later section.

\section{Two-Frequency Cooperative Two-Photon Absorption}

The transition matrix element for double-beam cooperative two-photon absorption can be obtained by setting $A \equiv B$ and $\alpha \equiv \beta$ in Eq. (5.13), and discarding the forbidden contributions associated with the third and fourth terms. The vectors $\mathbf{R}_{A}$ and $\mathbf{R}_{B}$ now become $\mathbf{R}_{A 1}$ and $\mathbf{R}_{A 2}$, leading to a matrix element given by;

$$
\begin{aligned}
M_{f i}= & K e_{1 i} e_{2 j}\left\{S_{i k}^{\alpha 0}\left(\omega_{1}\right) S_{j l}^{\alpha 0}\left(\omega_{2}\right) V_{k l}\left(\left[\omega_{\alpha 0}-\omega_{2}\right], \mathbf{R}\right) \exp \left(i\left[\mathbf{k}_{1} \cdot \mathbf{R}_{A 1}+\mathbf{k}_{2} \cdot \mathbf{R}_{A 2}\right]\right)\right. \\
& \left.+S_{i k}^{\alpha 0}\left(\omega_{1}\right) S_{j l}^{\alpha 0}\left(\omega_{2}\right) V_{k l}\left(\left[\omega_{\alpha 0}-\omega_{1}\right], \mathbf{R}\right) \exp \left(:\left[\mathbf{k}_{2} \cdot \mathbf{R}_{A 1}+\mathbf{k}_{1} \cdot \mathbf{R}_{A 2}\right]\right)\right\}
\end{aligned}
$$


Incorporating this into the Fermi Golden Rule yields a rate given by

$$
\begin{aligned}
\Gamma_{2 c}= & \left(2 \pi \rho_{f} / \hbar\right) K^{2} e_{1 i} e_{2 j} \bar{e}_{1 m} \bar{e}_{2 n} S_{i k}^{\alpha 0}\left(\omega_{1}\right) S_{j l}^{\alpha 0}\left(\omega_{2}\right) \bar{S}_{m o}^{\alpha 0}\left(\omega_{1}\right) \bar{S}_{n p}^{\alpha 0}\left(\omega_{2}\right) \\
& \times\left\{V_{k l}\left(\left[\omega_{\alpha 0}-\omega_{2}\right], \mathbf{R}\right) \bar{V}_{o p}\left(\left[\omega_{\alpha 0}-\omega_{2}\right], \mathbf{R}\right)\right. \\
& +V_{k l}\left(\left[\omega_{\beta 0}-\omega_{1}\right], \mathbf{R}\right) \bar{V}_{o p}\left(\left[\omega_{\beta 0}-\omega_{1}\right], \mathbf{R}\right) \\
& +V_{k l}\left(\left[\omega_{\alpha 0}-\omega_{2}\right], \mathbf{R}\right) \bar{V}_{o p}\left(\left[\omega_{\beta 0}-\omega_{1}\right], \mathbf{R}\right) \exp \left(i \mathbf{R} \cdot\left(\mathbf{k}_{2}-\mathbf{k}_{1}\right)\right) \\
& \left.+V_{k l}\left[\left[\omega_{\beta 0}-\omega_{1}\right], \mathbf{R}\right) \bar{V}_{o p}\left(\left[\omega_{\alpha 0}-\omega_{2}\right], \mathbf{R}\right) \exp \left(-i \mathbf{R} \cdot\left(\mathbf{k}_{2}-\mathbf{k}_{1}\right)\right)\right\}
\end{aligned}
$$

Using similar arguments to those outlined above, $K^{2}$ can be expressed in terms of the mean irradiances $\bar{I}_{1}$ and $\bar{I}_{2}$ of the two laser beams as follows:

$$
K^{2}=\left(1 / 4 c^{2} \varepsilon_{0}^{2}\right) \bar{I}_{1} \bar{I}_{2}
$$

and it is evident that there is no explicit dependence on the coherence properties of the sources. This is based on the assumption that there is no coherence correlation between the two radiation modes absorbed by the sample, which will normally be the case where two laser beams are used for the excitation: this is another feature that distinguishes the process from the single-beam case.

\section{Two-Frequency Distributive Two-Photon Absorption}

Finally, the transition matrix element for double-beam distributive twophoton absorption can be obtained from the third and fourth terms in Eq. (5.13), leading to a matrix element

$$
\begin{aligned}
M_{f i}= & K e_{1 i} e_{2 j}\left\{\chi_{i j k}^{\alpha 0}\left(A_{1}\right) \mu_{l}^{\alpha 0}\left(A_{2}\right) V_{k l}\left(\omega_{\alpha 0}, \mathbf{R}\right)\right. \\
& \left.+\chi_{i j k}^{\alpha 0}\left(A_{2}\right) \mu_{l}^{\alpha 0}\left(A_{1}\right) \bar{V}_{k l}\left(\omega_{\alpha 0}, \mathbf{R}\right) \exp \left(i\left[\mathbf{k}_{1}+\mathbf{k}_{2}\right] \cdot \mathbf{R}\right)\right\}
\end{aligned}
$$

The rate is then given by

$$
\begin{aligned}
\Gamma_{2 d}= & \left(2 \pi \rho_{f} / \hbar\right) K^{2} e_{1 i} e_{2 j} \bar{e}_{1 m} \bar{e}_{2 n} \\
& \times\left\{\chi_{i j k}^{\alpha 0}\left(A_{1}\right) \mu_{l}^{\alpha 0}\left(A_{2}\right) \bar{\chi}_{m n o}^{\alpha 0}\left(A_{1}\right) \bar{\mu}_{p}^{\alpha 0}\left(A_{2}\right) V_{k l}\left(\omega_{\alpha 0}, \mathbf{R}\right) \bar{V}_{o p}\left(\omega_{\alpha 0}, \mathbf{R}\right)\right. \\
& +\chi_{i j k}^{\alpha 0}\left(A_{2}\right) \mu_{l}^{\alpha 0}\left(A_{1}\right) \bar{\chi}_{m n o}^{\alpha 0}\left(A_{2}\right) \bar{\mu}_{p}^{\alpha 0}\left(A_{1}\right) \bar{V}_{k l}\left(\omega_{\alpha 0}, \mathbf{R}\right) V_{o p}\left(\omega_{\alpha 0}, \mathbf{R}\right) \\
& +\chi_{i j k}^{\alpha 0}\left(A_{2}\right) \mu_{l}^{\alpha 0}\left(A_{1}\right) \bar{\chi}_{m n o}^{\alpha 0}\left(A_{1}\right) \bar{\mu}_{p}^{\alpha 0}\left(A_{2}\right) \bar{V}_{k l}\left(\omega_{\alpha 0}, \mathbf{R}\right) \\
& \times \bar{V}_{o p}\left(\omega_{\alpha 0}, \mathbf{R}\right) \exp \left(i\left(\mathbf{k}_{1}+\mathbf{k}_{2}\right) \cdot \mathbf{R}\right) \\
& +\chi_{i j k}^{\alpha 0}\left(A_{1}\right) \mu_{l}^{\alpha 0}\left(A_{2}\right) \bar{\chi}_{m n o}^{\alpha 0}\left(A_{2}\right) \bar{\mu}_{p}^{\alpha 0}\left(A_{1}\right) V_{k l}\left(\omega_{\alpha 0}, \mathbf{R}\right) \\
& \left.\times V_{o p}\left(\omega_{\alpha 0}, \mathbf{R}\right) \exp \left(-i\left(\mathbf{k}_{1}+\mathbf{k}_{2}\right) \cdot \mathbf{R}\right)\right\}
\end{aligned}
$$

where once again the factor $K^{2}$ is as given in (6.10). The rate expressions 
given above represent the pre-averaged results. That is, they are directly applicable to systems where the two centers participating in the absorption process are rigidly held in fixed orientation witi respect to the laser beam(s) and with respect to one another. These results: are also a suitable starting point for the derivation of rate expressions applicable to fluid systems, where each center is free to rotate with respect to the beam(s) and also with respect to each other. This requires a series of rotationa averaging procedures which are described in detail in the next section.

\section{RATE EQUATIONS FOR FLUID MEDIA}

In fluid phase studies of mean-frequency absorption, it is necessary to take account of the effect of molecular tumbling on the rate of photoabsorption. Assuming that rotational structure is not resolvable, as will be the case in liquids, the necessary rotational averaging can be performed using a classical procedure based on the ergodic theorem. We first consider the case in which the relative orientation of the two centers $A$ and $B$ is fixed, but the $A-B$ system is free to move in the laser beam or beams. The initial result thereby obtained is then applicable to van der Waals rnolecules, and to polyatomic compounds in which A and B represent independent chromophores. The results may also be extended to solutions in an obvious way, thus describing coordination shell interactions between solute and solvent molecules through the addition of contributions relating to each $\mathbf{A}-\mathbf{B}$ pair. To derive results that ape appropriate for any such systems, it is necessary to perform a rotational average of the rate equations given in Section VI.

This principal rotational average is accomplished by first defining two Cartesian reference frames: one is a laboratory'fixed frame, (denoted by $p$ ), in which the laser polarization and propagation vectors are fixed, and the second frame (denoted by $a$ ), in which the molecular tensors and the vector $\mathbf{R}$ are fixed, is located on the A-B system. For convenience, the $a$ frame is defined as having its origin at center $\mathbf{A}$. The specific type of rotational average required depends on the exact nature of the pre-averaged result. For terms which do not carry an exponential phase factor, straightforward tensor averaging methods are employed (Andrews and Thirunamachandran 1977). However, where a phase factor persists in the result, a phased tensor averaging procedure is required (Andrews and Harlow 1984b). This reflects the fact that the phase of the laser light will generally be different at the two absorbing centers.

\section{A. Single-Frequency Cooperative Two-Photon Absorption}

The simplest case is that of single-beam cooperative two-photon absorption, which is the only process where no phased ave:ages arise. The pre-averaged rate, which is suitable for two centers that are rigldly held in a fixed orientation 
with respect to the laser beam and with respect to each other, has been given earlier as Eq. (6.3). Setting this equation in the two reference frames $a$ and $p$ defined above leads to

$$
\begin{aligned}
\Gamma_{1 c}= & \left(2 \pi \rho_{f} / \hbar\right) K^{\prime 2}\left\{e_{p_{i}} e_{p_{j}} \bar{e}_{p_{m}} \bar{e}_{p_{n}} l_{p_{i} a_{i}} l_{p_{j} a_{j}} l_{p_{m} a_{m}} l_{p_{n} a_{n}}\right. \\
& \times S_{a_{i} a_{k}}^{\alpha 0}(\omega) S_{a_{j} a_{l}}^{\beta 0}(\omega) \bar{S}_{a_{m} a_{o}}^{\alpha 0}(\omega) \bar{S}_{a_{n} a_{p}}^{\beta 0}(\omega) \\
& \left.\times V_{a_{k} a_{l}}\left(\left[\omega_{\beta 0}-\omega\right], \mathbf{R}\right) \bar{V}_{a_{o} a_{p}}\left(\left[\omega_{\beta 0}-\omega\right], \mathbf{R}\right)\right\}
\end{aligned}
$$

where the first letter of each pair of indices denotes the frame in which the following index is set, and $l_{p_{\alpha} a_{\alpha}}$ is the direction cosine between the $p_{\alpha}$ and $a_{\alpha}$ directions. Now, since all the vectors and tensors are referred to Cartesian frames in which they are invariant with respect to molecular rotation, the rotational averaging may be effected by averaging over the direction cosines alone. The required result for this calculation is (Andrews and Thirunamachandran 1977)

$$
\begin{gathered}
\left\langle l_{p_{i} a_{i}} l_{p_{j} a_{j} l_{p_{m} a_{m}} l_{p_{n} a_{n}}} l^{T}\left[\begin{array}{ll}
\delta_{p_{i} p_{j}} & \delta_{p_{m} p_{n}} \\
\delta_{p_{i} p_{m}} & \delta_{p_{j} p_{n}} \\
\delta_{p_{i} p_{n}} & \delta_{p_{j} p_{m}}
\end{array}\right]\left[\begin{array}{rrr}
4 & -1 & -1 \\
-1 & 4 & -1 \\
-1 & -1 & 4
\end{array}\right]\left[\begin{array}{ll}
\delta_{a_{i} a_{j}} & \delta_{a_{m} a_{n}} \\
\delta_{a_{i} a_{m}} & \delta_{a_{j} a_{n}} \\
\delta_{a_{i} a_{n}} & \delta_{a_{j} a_{m}}
\end{array}\right]\right.
\end{gathered}
$$

Application of this result to Eq. (7.1) yields

$$
\begin{aligned}
\left\langle\Gamma_{1 c}\right\rangle= & \left(\pi \rho_{f} / 15 \hbar\right) K^{2} V_{k l}\left(\left[\omega_{\beta 0}-\omega\right], \mathbf{R}\right) \bar{V}_{o p}\left(\left[\omega_{\beta 0}-\omega\right], \mathbf{R}\right) \\
& \times\left\{(4 \eta-2) S_{i k}^{\alpha 0}(\omega) S_{i l}^{\beta 0}(\omega) \bar{S}_{j o}^{\alpha 0}(\omega) \bar{S}_{j p}^{\beta 0}(\omega)\right. \\
& +(3-\eta) S_{i k}^{\alpha 0}(\omega) S_{j l}^{\beta 0}(\omega) \bar{S}_{i o}^{\alpha 0}(\omega) \bar{S}_{j p}^{\beta 0}(\omega) \\
& \left.+(3-\eta) S_{i k}^{\alpha 0}(\omega) S_{j l}^{\beta 0}(\omega) \bar{S}_{j o}^{\alpha 0}(\omega) \bar{S}_{i p}^{\beta 0}(\omega)\right\}
\end{aligned}
$$

where the brackets around $\Gamma_{1 c}$ signify the averaged result, and where

$$
\eta=(\mathbf{e} \cdot \mathbf{e})(\overline{\mathbf{e}} \cdot \overline{\mathbf{e}})
$$

The parameter $\eta$ takes the limiting values $\eta=0$ for circularly polarized light and $\eta=1$ for plane polarized light. In Eq. (7.3) and subsequently, the first of each pair of tensor indices, i.e., the one which indicates in which frame the particular tensor is rotationally invariant, has been dropped since all the indices are now related to the same system frame.

Equation (7.3) is the simplest of the results for synergistic photoabsorption presented in this review. The corresponding results for the three remaining mechanisms are appreciably more complex, and are more conveniently 
written using a shorthand notation. For consistency, it is therefore appropriate to re-express Eq. (7.3) in terms of "his generalized notation as follows;

$$
\left\langle\Gamma_{1 c}\right\rangle=\left(2 \pi \rho_{f} / \hbar\right) K^{\prime 2} \sum_{p, q} g_{(4 ; 0)}^{p q} A^{\prime(0 ; p)} T_{c}^{(0 ; q)}\left(\alpha, \beta, \alpha, \beta ; \omega, \omega, \omega, \omega ; \omega_{\beta 0}-\omega, \omega_{\beta 0}-\omega\right)
$$

where the $g_{(4 ; 0)}^{p q}$ are numerical coefficients derived from the theory of tensor averaging (Andrews and Ghoul 1982), and $A^{\prime}, T_{c}$ are the specific polarization and molecular tensor parameters for this particular mechanism. In general these are defined by:

and

$$
A^{\prime(j ; p)}(\hat{\mathbf{u}})=e_{1 i} e_{2 j} \bar{e}_{1 k} \bar{e}_{2 l} U_{i j k !}^{(4 ; j ; p)}(\hat{\mathbf{u}})
$$

$$
\begin{aligned}
T_{c}^{(j ; q)}\left(\alpha, \beta, \alpha^{\prime}, \beta^{\prime} ; \omega_{1}, \omega_{2}, \omega_{1}^{\prime}, \omega_{2}^{\prime} ; \omega_{\alpha o}-\omega_{1}, \omega_{\alpha^{\prime} 0}-\omega_{1}^{\prime}\right) \\
=S_{\lambda \pi}^{\alpha 0}\left(\omega_{1}\right) S_{\mu \rho}^{\beta 0}\left(\omega_{2}\right) \bar{S}_{v \sigma}^{\alpha^{\prime} 0}\left(\omega_{1}^{\prime}\right) \bar{S}_{o \tau}^{\beta^{\prime} 0}\left(\omega_{2}^{\prime}\right) \\
\quad \times V_{\pi \rho}\left(\left[\omega_{\alpha 0}-\omega_{1}\right], \mathbf{R}\right) \bar{V}_{\sigma \tau}\left(\left[\omega_{\alpha^{\prime} 0}-\omega_{1}^{\prime}\right], \mathbf{R}\right) W_{\lambda \mu v o}^{(\mathbf{4} ; j ; q)}(\hat{\mathbf{R}})
\end{aligned}
$$

where $U_{i j k l}^{(4 ; j ; p)}(\hat{\mathbf{u}})$ and $W_{\lambda \mu v o}^{(4 ; j ; q)}(\hat{\mathbf{R}})$ are irreducible tensor projections (Andrews and Harlow 1984b) and the explicit results for $A^{\prime}$ and $T_{c}$ are presented in Tables I and II.

The result, Eq. (7.5), is directly applicable to the case of single-beam cooperative two-photon absorption where the mutual orientation of $A$ and B is fixed, but the A-B system may rotate with respect to the laser beam (the rotating pair case). We now consider the situation in which the two molecules involved in the interaction are free to take up any separation and mutual orientation; this may be termed the free molecules case. Two further averages are now required to derive a suitable rate expression. The first step involves specifying a molecule-fixed Cartesian reference frame $b$ in which the molecular tensors of center $B$ are rotationally invariant, and then performing a rotational average with respect to the $a$ frame. This step accounts for the rotation of center $\mathbf{B}$ relative to center $\mathbf{A}$. The second step is carried out by specifying an $r$ frame in which the $\mathbf{R}$ vectors are rotationally invariant, and subsequently averaging over the orientation of this frame with respect to the $a$ frame; this step accounts for the random orientation of the vector $\mathbf{A B}$ relative to center $\mathbf{A}$.

It is worth noting that this full triple-averaging procedure is required to account for any fluid sample composed of randomily orientated free molecules; the overall procedure, due to Schipper (1981), is summarized in Table III. Although each of the three stages of the averagirg procedure is essential, the order in which they are conducted in a particular case is not important. 
TABLE I

Explicit Form of the Polarization Parameters $A^{\prime(j ; p)}(\hat{\mathbf{u}})$

\begin{tabular}{lll}
\hline$j$ & $p$ & $A^{\prime(j ; p)}$ \\
\hline 0 & 1 & $(\mathbf{e} \cdot \mathbf{e})(\overline{\mathbf{e}} \cdot \overline{\mathbf{e}})$ \\
0 & 2 & 1 \\
0 & 3 & 0 \\
1 & 1 & $(\mathbf{e} \times \overline{\mathbf{e}}) \cdot \hat{\mathbf{u}}$ \\
1 & 2 & $(\mathbf{e} \times \overline{\mathbf{e}}) \cdot \hat{\mathbf{u}}$ \\
1 & 3 & $(\mathbf{e} \times \overline{\mathbf{e}}) \cdot \hat{\mathbf{u}}$ \\
1 & 4 & $(\mathbf{e} \times \overline{\mathbf{e}}) \cdot \hat{\mathbf{u}}$ \\
1 & 5 & 0 \\
1 & 6 & $\left(-\frac{1}{3}\right)(\mathbf{e} \cdot \mathbf{e})(\overline{\mathbf{e}} \cdot \overline{\mathbf{e}})$ \\
2 & 1 & $-\frac{1}{3}$ \\
2 & 2 & $-\frac{1}{3}$ \\
2 & 3 & $-\frac{1}{3}$ \\
2 & 4 & $-\frac{1}{3}$ \\
2 & 5 & $\left(-\frac{1}{3}\right)(\mathbf{e} \cdot \mathbf{e})(\overline{\mathbf{e}} \cdot \overline{\mathbf{e}})$ \\
2 & 6 & $\left(-\frac{1}{5}\right)(\mathbf{e} \times \overline{\mathbf{e}}) \cdot \hat{\mathbf{u}}$ \\
3 & 1 & $\left(-\frac{1}{5}\right)(\mathbf{e} \times \overline{\mathbf{e}}) \cdot \hat{\mathbf{u}}$ \\
3 & 2 & 0 \\
3 & 3 & $\left(\frac{1}{35}\right)[(\mathbf{e} \cdot \mathbf{e})(\overline{\mathbf{e}} \cdot \overline{\mathbf{e}})+2]$ \\
4 & 1 &
\end{tabular}

TABLE II

Explicit Form of Cooperative Molecular Invariants ${ }^{a}$

\begin{tabular}{|c|c|c|}
\hline$j$ & $q$ & $Z_{\pi \rho \sigma r}^{(j ; q)}$ \\
\hline 0 & 1 & $S_{\lambda \pi}^{\alpha 0}\left(\omega_{1}\right) S_{\mu \rho}^{\beta 0}\left(\omega_{2}\right) \bar{S}_{\mu \sigma}^{\alpha^{\prime} 0}\left(\omega_{1}^{\prime}\right) \bar{S}_{\mu \tau}^{\beta^{\prime} 0}\left(\omega_{2}^{\prime}\right)$ \\
\hline 0 & 2 & $S_{\lambda \pi}^{\alpha 0}\left(\omega_{1}\right) S_{\mu \rho}^{\beta \rho}\left(\omega_{2}\right) \bar{S}_{\lambda \sigma}^{\alpha^{\prime} 0}\left(\omega_{1}^{\prime}\right) \bar{S}_{\mu \tau}^{\beta^{\prime} \mathrm{O}}\left(\omega_{2}^{\prime}\right)$ \\
\hline 0 & 3 & $S_{\lambda \pi}^{\alpha 0}\left(\omega_{1}\right) S_{\mu \rho}^{\beta 0}\left(\omega_{2}\right) \bar{S}_{\mu \sigma}^{a^{\prime} 0}\left(\omega_{1}^{\prime}\right) \bar{S}_{\lambda \tau}^{\beta^{\prime} 0}\left(\omega_{2}^{\prime}\right)$ \\
\hline 1 & 1 & $\varepsilon_{\lambda \mu \nu} \hat{R}_{v} S_{\lambda \pi}^{\alpha 0}\left(\omega_{1}\right) S_{\mu \rho}^{\beta O}\left(\omega_{2}\right) \bar{S}_{o \sigma}^{a^{\prime} 0}\left(\omega_{1}^{\prime}\right) \bar{S}_{o r}^{\beta^{\prime} 0}\left(\omega_{2}^{\prime}\right)$ \\
\hline 1 & 2 & $\varepsilon_{\lambda \mu \nu} \hat{R}_{v} S_{\lambda \pi}^{\alpha 0}\left(\omega_{1}\right) S_{o p}^{\beta \sigma}\left(\omega_{2}\right) \bar{S}_{\mu \sigma}^{\alpha^{\prime} 0}\left(\omega_{1}^{\prime}\right) \bar{S}_{o \tau}^{\beta^{\prime} 0}\left(\omega_{2}^{\prime}\right)$ \\
\hline 1 & 3 & $\varepsilon_{\lambda \mu \nu} \hat{R}_{v} S_{\lambda \pi}^{\alpha 0}\left(\omega_{1}\right) S_{o p}^{\beta 0}\left(\omega_{2}\right) \bar{S}_{o \sigma}^{\alpha^{\prime} 0}\left(\omega_{1}^{\prime}\right) \bar{S}_{\mu \tau}^{\beta^{\prime} 0}\left(\omega_{2}^{\prime}\right)$ \\
\hline 1 & 4 & $\varepsilon_{\lambda \mu v} \hat{R}_{v} S_{o \pi}^{\alpha 0}\left(\omega_{1}\right) S_{\lambda \rho}^{\beta 0}\left(\omega_{2}\right) \bar{S}_{\mu \sigma}^{\alpha^{\prime} 0}\left(\omega_{1}^{\prime}\right) \bar{S}_{o \tau}^{\beta^{\prime} 0}\left(\omega_{2}^{\prime}\right)$ \\
\hline 1 & 5 & $\varepsilon_{\lambda \mu \nu} \hat{R}_{v} S_{o \pi}^{\alpha 0}\left(\omega_{1}\right) S_{\lambda \rho}^{\beta 0}\left(\omega_{2}\right) \bar{S}_{o \sigma}^{\alpha^{\prime} 0}\left(\omega_{1}^{\prime}\right) \bar{S}_{\mu \tau}^{\beta^{\prime} 0}\left(\omega_{2}^{\prime}\right)$ \\
\hline 1 & 6 & $\varepsilon_{\lambda \mu v} \hat{R}_{v} S_{o \pi}^{\alpha 0}\left(\omega_{1}\right) S_{o \rho}^{\beta 0}\left(\omega_{2}\right) \bar{S}_{\lambda \sigma}^{\alpha^{\prime} 0}\left(\omega_{1}^{\prime}\right) \bar{S}_{\mu \tau}^{\beta^{\prime} 0}\left(\omega_{2}^{\prime}\right)$ \\
\hline 2 & 1 & $\begin{array}{l}\hat{R}_{\mu} \hat{R}_{v} S_{\lambda \pi}^{\alpha O}\left(\omega_{1}\right) S_{\lambda \rho}^{\beta O}\left(\omega_{2}\right) \bar{S}_{\mu \sigma}^{\alpha^{\circ} 0}\left(\omega_{1}^{\prime}\right) \bar{S}_{v v}^{\beta \prime O}\left(\omega_{2}^{\prime}\right) \\
-\left(\frac{1}{3}\right) S_{\lambda \pi}^{a 0}\left(\omega_{1}\right) S_{\lambda \rho}^{\beta O}\left(\omega_{2}\right) \bar{S}_{\mu \sigma}^{\alpha^{\prime} 0}\left(\omega_{1}^{\prime}\right) \bar{S}_{\mu \tau}^{\beta^{\prime O} 0}\left(\omega_{2}^{\prime}\right)\end{array}$ \\
\hline 2 & 2 & $\begin{array}{l}\hat{R}_{\mu} \hat{R}_{v} S_{\lambda \pi}^{\alpha 0}\left(\omega_{1}\right) S_{\mu \rho}^{\beta 0}\left(\omega_{2}\right) \bar{S}_{\lambda \sigma}^{\alpha^{\circ} 0}\left(\omega_{1}^{\prime}\right) \bar{S}_{v \tau}^{\beta^{\prime} 0}\left(\omega_{2}^{\prime}\right) \\
-\left(\frac{1}{3}\right) S_{\lambda \pi}^{\alpha 0}\left(\omega_{1}\right) S_{\mu \rho}^{\beta 0}\left(\omega_{2}\right) \bar{S}_{\lambda \sigma}^{\alpha 0}\left(\omega_{1}^{\prime}\right) \bar{S}_{v \tau}^{\beta^{\prime 0} 0}\left(\omega_{2}^{\prime}\right)\end{array}$ \\
\hline 2 & 3 & $\begin{array}{l}\hat{R}_{\mu} \hat{R}_{v} S_{\lambda \pi}^{\alpha 0}\left(\omega_{1}\right) S_{\mu \rho}^{\beta 0}\left(\omega_{2}\right) \bar{S}_{v \sigma}^{\alpha \sigma}\left(\omega_{1}^{\prime}\right) \bar{S}_{\lambda \tau}^{\beta^{\prime 0} 0}\left(\omega_{2}^{\prime}\right) \\
-\left(\frac{1}{3}\right) S_{\lambda \pi}^{\alpha 0}\left(\omega_{1}\right) S_{\mu \rho}^{\beta 0}\left(\omega_{2}\right) \bar{S}_{\mu \sigma}^{\alpha^{\prime} 0}\left(\omega_{1}^{\prime}\right) \bar{S}_{\lambda \tau}^{\beta^{\prime 0} 0}\left(\omega_{2}^{\prime}\right)\end{array}$ \\
\hline
\end{tabular}


TABLE II (Continued)

\begin{tabular}{|c|c|c|}
\hline$j$ & $q$ & $Z_{\pi p \sigma t}^{(j, q)}$ \\
\hline 2 & 4 & 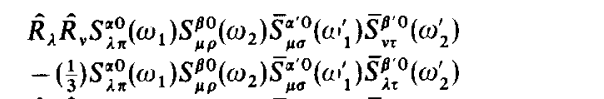 \\
\hline 2 & 5 & 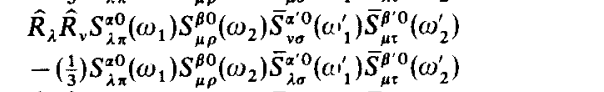 \\
\hline 2 & 6 & 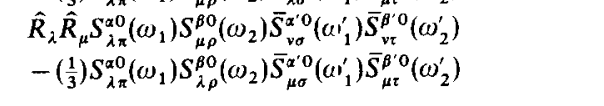 \\
\hline 3 & 1 & 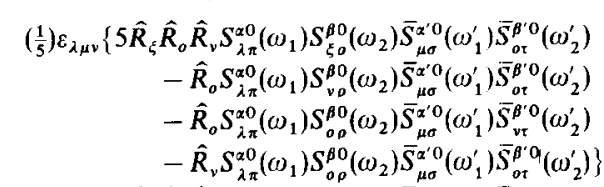 \\
\hline 3 & 2 & 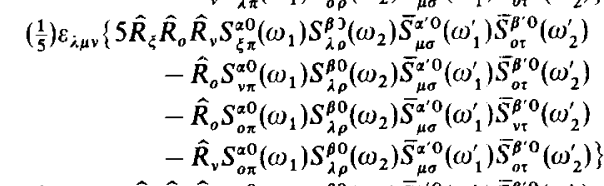 \\
\hline 3 & 3 & 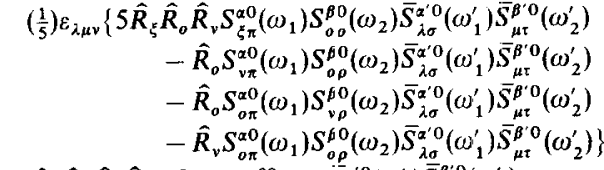 \\
\hline 4 & 1 & 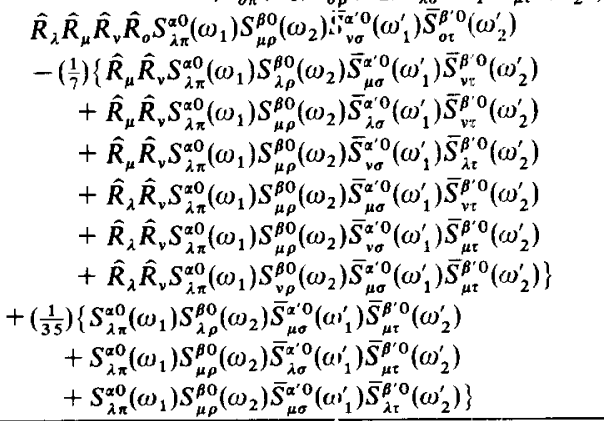 \\
\hline
\end{tabular}

${ }^{a}$ Defined by

$$
\begin{aligned}
T_{c}^{(j ; q)}\left(\alpha, \beta, \alpha^{\prime}, \beta^{\prime} ; \omega_{1}, \omega_{2}, \omega_{1}^{\prime}, \omega_{2}^{\prime} ; \omega_{\alpha 0}-\omega_{1}, \omega_{\alpha^{\prime} 0}-\omega_{1}^{\prime}\right) \\
=S_{\lambda \pi}^{\alpha 0}\left(\omega_{1}\right) S_{\mu \rho}^{\beta 0}\left(\omega_{2}\right) \bar{S}_{v \sigma}^{a^{\prime} 0}\left(\omega_{1}^{\prime}\right) \bar{S}_{o \tau}^{\beta^{\prime} 0}\left(\omega_{2}^{\prime}\right) \\
\quad \times V_{\pi \rho}\left(\left[\omega_{\alpha 0}-\omega_{1}\right], \mathbf{R}\right) \bar{V}_{\sigma \tau}\left(\left[\omega_{\alpha^{\prime} 0}-\omega_{1}^{\prime}\right], \mathbf{R}\right) W_{\lambda \mu v o}^{(4 ; j ; q)}(\hat{\mathbf{R}})
\end{aligned}
$$

and here given by

$$
T_{c}^{(j ; q)}=Z_{\pi \rho \sigma \tau}^{(j ; q)} V_{\pi \rho}\left(\left[\omega_{a 0}-\omega_{1}\right], \mathbf{R}\right) \vec{V}_{\sigma \tau}\left(\left[\omega_{\alpha^{\prime} 0}-\omega_{1}^{\prime}\right], \mathbf{R}\right)
$$


TABLE III

Rotational Averaging Scheme ${ }^{a}$

\begin{tabular}{lccccc}
\hline Vector and tensor quantities & $e$ & $\left(S^{\alpha 0}, X^{\alpha 0}, \mu^{\alpha 0}\right)$ & $\left(S^{\beta 0}, X^{\beta 0}, \mu^{\beta 0}\right)$ & $V$ & Result \\
\hline Pre-averaging & $p$ & $p$ & $p$ & $p$ & \\
Rotational averaging $a \leftrightarrow p$ & $\downarrow$ & $\downarrow$ & $\downarrow$ & $\downarrow$ & \\
& $\downarrow$ & $a$ & $a$ & $a$ & Rotating pair \\
Rotational averaging $b \leftrightarrow a$ & $p$ & $a$ & $\downarrow$ & $\downarrow$ & \\
Rotational averaging $r \leftrightarrow a$ & $\downarrow$ & $\downarrow$ & $b$ & $a$ & \\
\hline
\end{tabular}

'The Cartesian reference frames $p, a, b$, and $r$ are defined as follows: $p$ denotes a laboratory-fixed frame in which the laser polarization vectors are fixed; $a$ signifies a frame of reference that has been chosen to have its origin at center $A$; similarly $b$ has its origin at center $B ; r$ represents a frame in which the $\mathbf{R}$ vectors are rotationally invariant.

Ultimately, equivalent rate expressions result regardless of the order in which the averages are calculated. For instance, it is possible to conduct an alternative series of rotational averages in which first $A$ and $B$ are averaged with respect to $\mathbf{R}$, and then $\mathbf{R}$ is averaged with respect to the laboratory frame. In performing the lengthy calculations associated with this work, it is useful to have the option to use whichever route proves to be shorter, and least mathematically complex, for the particular case under study. Applying such procedures to Eq. (7.5) gives the rate of single-beam cooperative two-photon absorption for a completely fluid sample with no molecular orientational correlation. The result is an equation that can be written in exactly the same form as Eq. (7.5), but where each $T_{c}^{(j ; q)}$ is replaced by its fully averaged counterpart $\left\langle T_{c}(j ; q) 》\right.$. It is worth noting that this procedure has no effect on the polarization dependence, which is entirely determined by the parameters $A^{\prime}$.

While a similar method of calculation is required for each of the processes under consideration, important differences arise in all other cases because an exponential phase factor persists in the pre-averaged results. This leads to the necessity of employing phased rotational averaging. Such averaging procedures are described elsewhere, and for a detailed discussion of the subject the reader is referred to Andrews and Harlow (1984b). Results are given below for both rotating pairs and free molecules undergoing bimolecular mean-frequency absorption by each of the three remaining mechanisms.

\section{B. Single-Frequency Distributive Two-Photon Absorption}

To obtain a result applicable to a rotating pair requires the performance of a rotational average on Eq. (6.7). Whereas the first two terms of this equation require a straightforward fourth rank average, terms three and four require 
a phased fourth rank average. The result for the pair rate may be expressed as follows;

$$
\begin{aligned}
\left\langle\Gamma_{1 d}\right\rangle= & \left(\frac{2 \pi \rho_{f}}{\hbar}\right) K^{\prime 2}\left\{\sum_{p, q} g_{(4 ; 0)}^{p q} A^{\prime(0 ; p)}(\hat{\mathbf{s}}) T_{d}^{(0 ; q)}\left(\iota, \beta, \alpha, \beta ; A, B, A, B ; \omega_{\beta 0}, \omega_{\beta 0}\right)\right. \\
& +\sum_{j=0} \sum_{p, q} \frac{(2 j) !}{2^{j}(j !)^{2}} i^{j} j_{j}(|\mathbf{s}| R) g_{(4, j)}^{p q} A^{\prime(j ; p)}(\hat{\mathbf{s}}) \\
& \left.\times T_{d}^{(j ; q)}\left(\alpha, \beta, \beta, \alpha ; A, B, B, A ; \omega_{\beta 0},-\omega_{c: 0}\right)\right\} \\
& +\left\{\alpha, A,-\omega_{\alpha 0} \leftrightarrow \beta, B, \omega_{\beta 0}\right\}
\end{aligned}
$$

where $s$ is the wave-vector sum for the two ab:orbed photons $(=2 k)$, and

$$
\begin{aligned}
& T_{d}^{(j ; q)}\left(\alpha, \beta, \alpha^{\prime}, \beta^{\prime} ; A_{1}, A_{2}, A_{3}, A_{4} ; \omega_{\alpha 0}, \omega_{\alpha 0}^{\prime}\right) \\
& \quad=\chi_{\lambda \mu \pi}^{\alpha 0}\left(A_{1}\right) \mu_{\rho}^{\beta 0}\left(A_{2}\right) \bar{\chi}_{v o \sigma}^{\alpha^{\prime} 0}\left(A_{3}\right) \bar{\mu}_{\tau}^{\beta^{\prime} 0}\left(A_{4}\right) V_{\pi \rho}\left(\omega_{\alpha 0}, \mathbf{R}\right) \bar{V}_{\sigma \tau}\left(\omega_{k 0}^{\prime}, \mathbf{R}\right) W_{\lambda \mu v o}^{(4 ; j ; q)}(\hat{\mathbf{R}})
\end{aligned}
$$

where the $j_{n}$ denote spherical Bessel functions of order $n$, and the general form of the molecular parameters $T_{d}$ is given in Table IV. As is evident on inspection of the polarization parameters $A^{\prime(j ; z)}(\hat{\mathbf{s}})$ in Table 1 , remembering that $\hat{\mathbf{s}}=\widehat{\mathbf{k}}$, the involvement of terms with $j \neq 0$ in the rate equation leads to a dependence on the two variables $\eta=(\mathbf{e} \cdot \mathbf{e})(\overline{\mathbf{e}} \cdot \overline{\mathbf{e}})$, and $\zeta=(\mathbf{e} \times \overline{\mathbf{e}}) \cdot \overline{\mathbf{k}}$, the relationship between which is discussed in Section IX. Once again, the corresponding rate equation for the case of a ferfect fluid consisting of free molecules is obtained by carrying out two further averages on each molecular term in the above expression.

\section{Two-Frequency Cooperative Two-Photon Absorption}

For two-frequency excitation, the averaged rest.lt for a rotating pair is given by

$$
\begin{aligned}
\left\langle\Gamma_{2 \mathbf{c}}\right\rangle= & \left(\frac{2 \pi \rho_{f}}{\hbar}\right) K^{2}\left\{\sum_{p, q} g_{(4 ; 0)}^{p q} A^{(0 ; p)}(\hat{\mathbf{u}})\right. \\
& \times T_{c}^{(0 ; q)}\left(\alpha, \alpha, \alpha, \alpha ; \omega_{1}, \omega_{2}, \omega_{1}, \omega_{2} ; \omega_{\alpha 0}-\omega_{1}, \omega_{\alpha 0}-\omega_{1}\right) \\
& +\sum_{j=0} \sum_{p, q} \frac{(2 j) !}{2^{j}(j !)^{2}} i^{j} j_{j}(|\mathbf{u}| R) g_{(4, j)}^{p q} A^{(j ; p)}(\hat{\mathbf{u}}) \\
& \left.\times T_{c}^{(j ; q)}\left(\alpha, \alpha, \alpha, \alpha ; \omega_{1}, \omega_{2}, \omega_{1}, \omega_{2} ; \omega_{\alpha 0}-\omega_{1}, \omega_{\alpha 0}-\omega_{2}\right)\right\} \\
& +\left\{\omega_{\alpha 0}-\omega_{1} \leftrightarrow \omega_{\alpha 0}-\omega_{2}\right\}
\end{aligned}
$$


TABLE IV

Explicit Form of the Distributive Molecular Invariants ${ }^{a}$

\begin{tabular}{|c|c|c|}
\hline$j$ & $q$ & $Z_{\pi \rho \sigma \tau}^{(p ; q)}\left(A_{1}, A_{2}, A_{3}, A_{4}\right)$ \\
\hline 0 & 1 & $\chi_{\lambda \lambda \pi}^{\chi 0}\left(A_{1}\right) \mu_{\rho}^{\beta 0}\left(A_{2}\right) \bar{\chi}_{\mu \mu \sigma}^{\alpha^{\prime} 0}\left(A_{3}\right) \bar{\mu}_{\tau}^{\beta^{\prime} 0}\left(A_{4}\right)$ \\
\hline 0 & 2 & $\chi_{\lambda \mu \pi}^{\chi 0}\left(A_{1}\right) \mu_{\rho}^{\beta 0}\left(A_{2}\right) \bar{\chi}_{\lambda, \mu \sigma}^{\alpha^{\prime} 0}\left(A_{3}\right) \bar{\mu}_{\tau}^{\beta, 0}\left(A_{4}\right)$ \\
\hline 0 & 3 & $\chi_{\lambda \mu \pi}^{\chi 0}\left(A_{1}\right) \mu_{\rho}^{\beta 0}\left(A_{2}\right) \bar{\chi}_{\mu \alpha a}^{\alpha^{\prime} 0}\left(A_{3}\right) \bar{\mu}_{\tau}^{\beta^{\prime} 0}\left(A_{4}\right)$ \\
\hline 1 & 1 & $\varepsilon_{\lambda \mu \nu} \hat{R}_{v} \chi_{\lambda \mu \pi}^{\alpha 0}\left(A_{1}\right) \mu_{\rho}^{\beta 0}\left(A_{2}\right) \chi_{o o \sigma}^{\alpha^{\prime 0} 0}\left(A_{3}\right) \bar{\mu}_{\tau}^{\beta^{\prime} 0}\left(A_{4}\right)$ \\
\hline 1 & 2 & $\varepsilon_{\lambda \sigma v} \hat{R}_{\nu} \chi_{\lambda \mu \pi}^{\alpha 0}\left(A_{1}\right) \mu_{\rho}^{\beta 0}\left(A_{2}\right) \bar{\chi}_{\rho \mu \sigma}^{\bar{c}^{\prime} 0}\left(A_{3}\right) \bar{\mu}_{\tau}^{\beta^{\prime 0}}\left(A_{4}\right)$ \\
\hline 1 & 3 & $\varepsilon_{\lambda o v} \hat{R}_{v} \chi_{\lambda \mu \pi}^{a 0}\left(A_{1}\right) \mu_{\rho}^{\beta 0}\left(A_{2}\right) \bar{\chi}_{\mu \sigma \sigma}^{z^{\prime 0}}\left(A_{3}\right) \bar{\mu}_{\tau}^{\beta^{\prime} 0}\left(A_{4}\right)$ \\
\hline 1 & 4 & $\varepsilon_{\mu \nu v} \hat{R}_{v} \chi_{\lambda \mu \pi}^{\alpha 0}\left(A_{1}\right) \mu_{\rho}^{\beta 0}\left(A_{2}\right) \chi_{o \lambda \sigma}^{\alpha^{\prime 0} 0}\left(A_{3}\right) \bar{\mu}_{\tau}^{\beta^{\prime} 0}\left(A_{4}\right)$ \\
\hline 1 & 5 & $\varepsilon_{\mu \nu v} \hat{R}_{v} \chi_{\lambda \mu \pi}^{\alpha 0}\left(A_{1}\right) \mu_{\rho}^{\beta 0}\left(A_{2}\right) \bar{\chi}_{\lambda \sigma \sigma}^{\alpha^{\prime} 0}\left(A_{3}\right) \bar{\mu}_{\tau}^{\beta^{\prime} 0}\left(A_{4}\right)$ \\
\hline 1 & 6 & $\varepsilon_{\mu \nu v} \hat{R}_{v} \chi_{\lambda \lambda \pi}^{\alpha 0}\left(A_{1}\right) \mu_{\rho}^{\beta 0}\left(A_{2}\right) \bar{\chi}_{\mu o \sigma}^{\chi^{\prime} 0}\left(A_{3}\right) \bar{\mu}_{\tau}^{\beta, 0}\left(A_{4}\right)$ \\
\hline 2 & 1 & $\begin{array}{r}\hat{R}_{\mu} \hat{R}_{\nu} \chi_{\lambda \lambda \pi}^{\alpha 0}\left(A_{1}\right) \mu_{\rho}^{\beta 0}\left(A_{2}\right) \bar{\chi}_{\mu v \sigma}^{\alpha^{\prime} 0}\left(A_{3}\right) \bar{\mu}_{\tau}^{\beta^{\prime} 0}\left(A_{4}\right) \\
-\left(\frac{1}{3}\right) \chi_{\lambda \lambda \pi}^{\alpha 0}\left(A_{1}\right) \mu_{\rho}^{\beta 0}\left(A_{2}\right) \bar{\chi}_{\mu \mu \sigma}^{\alpha, 0}\left(A_{3}\right) \bar{\mu}_{\tau}^{\beta, 0}\left(A_{4}\right)\end{array}$ \\
\hline 2 & 2 & $\begin{array}{r}\hat{R}_{\mu} \hat{R}_{v} \chi_{\lambda \mu \pi}^{\alpha 0}\left(A_{1}\right) \mu_{\rho}^{\beta 0}\left(A_{2}\right) \bar{\chi}_{\lambda v \sigma}^{\chi^{\circ} 0}\left(A_{3}\right) \bar{\mu}_{\tau}^{\beta^{\circ} 0}\left(A_{4}\right) \\
-\left(\frac{1}{3}\right) \chi_{\lambda \mu \pi}^{\alpha 0}\left(A_{1}\right) \mu_{\rho}^{\beta 0}\left(A_{2}\right) \bar{\chi}_{\mu \mu \sigma}^{\alpha^{0}}\left(A_{3}\right) \bar{\mu}_{\tau}^{\beta^{\circ} 0}\left(A_{4}\right)\end{array}$ \\
\hline 2 & 3 & $\begin{array}{l}\hat{R}_{\mu} \hat{R}_{v} \chi_{\lambda_{\mu \pi}^{\alpha 0}}^{\alpha 0}\left(A_{1}\right) \mu_{\rho}^{\beta 0}\left(A_{2}\right) \bar{\chi}_{v \lambda \sigma}^{\alpha^{\prime} 0}\left(A_{3}\right) \bar{\mu}_{\tau}^{\beta^{\circ} 0}\left(A_{4}\right) \\
-\left(\frac{1}{3}\right) \chi_{\mu \mu \pi}^{\alpha 0}\left(A_{1}\right) \mu_{\rho}^{\beta 0}\left(A_{2}\right) \bar{\chi}_{\mu \lambda \sigma}^{\alpha^{\prime} 0}\left(A_{3}\right) \bar{\mu}_{\tau}^{\beta^{\prime} 0}\left(A_{4}\right)\end{array}$ \\
\hline 2 & 4 & 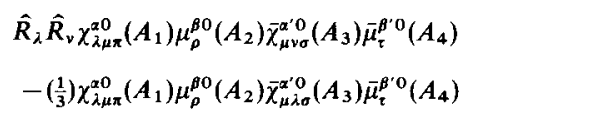 \\
\hline 2 & 5 & 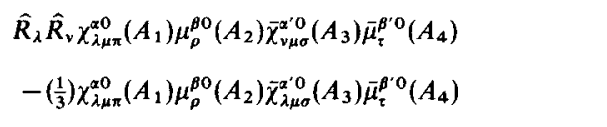 \\
\hline 2 & 6 & 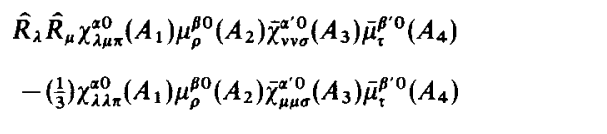 \\
\hline 3 & 1 & 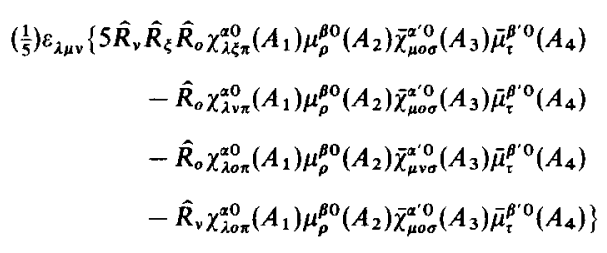 \\
\hline
\end{tabular}


TABLE IV (Continued)

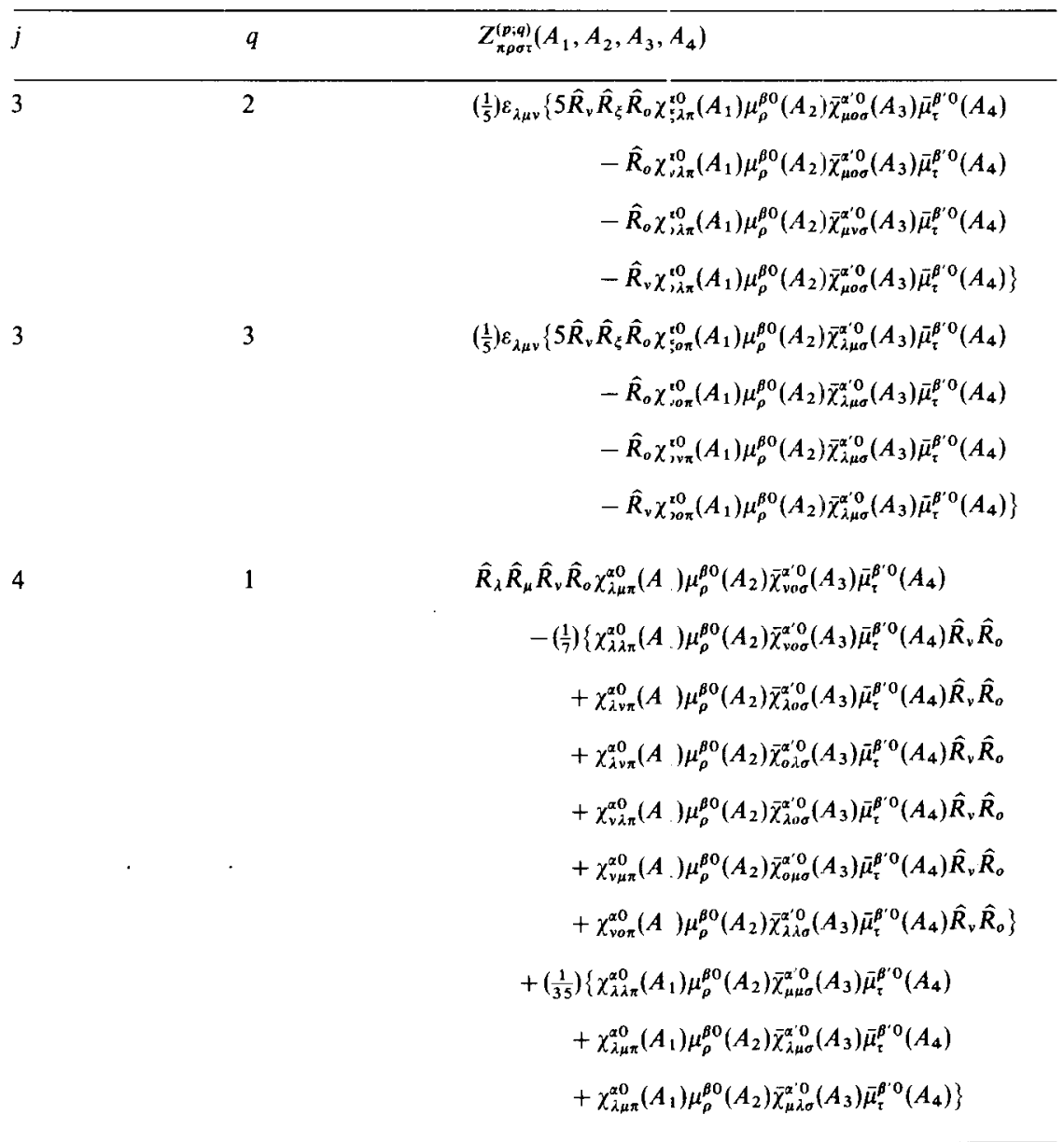

${ }^{a}$ Defined by

$$
\begin{aligned}
& T_{d}^{(j ; q)}\left(\alpha, \beta, \alpha^{\prime}, \beta^{\prime} ; A_{1}, A_{2}, A_{3}, A_{4} ; \omega_{\alpha 0}, \omega_{\alpha 0}\right) \\
& \quad=\chi_{\lambda \mu \pi}^{\alpha 0}\left(A_{1}\right) \mu_{\rho}^{\beta 0}\left(A_{2}\right) \bar{\chi}_{v o \sigma}^{\alpha^{\prime} 0}\left(A_{3}\right) \bar{\mu}_{\tau}^{\beta^{\prime} 0}\left(A_{4}\right) V_{\pi \rho}\left(\omega_{\alpha 0}, \mathbf{E}^{\prime}\right) \bar{V}_{a \mathrm{r}}\left(\omega_{\alpha 0}, \mathbf{R}\right) W_{\lambda \mu \nu 0}^{\left(\alpha_{j \mu j} ; q\right)}(\hat{\mathbf{R}}) .
\end{aligned}
$$

and here given by

$$
T_{d}^{(j ; q)}=Z_{\pi \rho \sigma \tau}^{(p ; q)} V_{\pi_{\rho}}\left(\omega_{x 0}, \mathbf{R}\right) \bar{V}_{\sigma \tau}\left(\alpha_{a 0}, \mathbf{R}\right)
$$


where the wave-vector difference $\mathbf{u}=\left(\mathbf{k}_{2}-\mathbf{k}_{1}\right)$ and

$$
A^{(j ; p)}(\hat{\mathbf{u}})=e_{1_{i}} e_{2_{j}} \bar{e}_{1_{k}} \bar{e}_{2_{l}} U_{i j k l}^{(4 ; j ; p)}(\hat{\mathbf{u}})
$$

the explicit values for which are given in Table V. As before, subsequent rotational averaging of the molecular parameters $T_{c}$ produces the result applicable to a system of randomly oriented molecules.

\section{Two-Frequency Distributive Two-Photon Absorption}

The results for the distributive case of two-photon absorption can be expressed in a similar manner. The polarization parameters $A^{(j ; p)}$ are identical in form to those tabulated in Table $\mathrm{V}$, and the wave-vector sum $\mathbf{s}$ is now

TABLE V

Explicit Form of the Polarization Parameters $A^{(j ; p)}(\hat{\mathbf{u}})$

\begin{tabular}{|c|c|c|}
\hline$j$ & $p$ & $A^{(j ; p)}(\hat{\mathbf{u}})$ \\
\hline 0 & 1 & $\left(\mathbf{e}_{1} \cdot \mathbf{e}_{2}\right)\left(\overline{\mathbf{e}}_{1} \cdot \overline{\mathbf{e}}_{2}\right)$ \\
\hline 0 & 2 & 1 \\
\hline 0 & 3 & $\left(\mathbf{e}_{1} \cdot \overline{\mathbf{e}}_{2}\right)\left(\mathbf{e}_{2} \cdot \overline{\mathbf{e}}_{1}\right)$ \\
\hline 1 & 1 & $\left(\overline{\mathbf{e}}_{1} \cdot \overline{\mathbf{e}}_{2}\right)\left(\mathbf{e}_{1} \times \mathbf{e}_{2}\right) \cdot \hat{\mathbf{u}}$ \\
\hline 1 & 2 & $\left(\mathbf{e}_{1} \times \overline{\mathbf{e}}_{1}\right) \cdot \hat{\mathbf{u}}$ \\
\hline 1 & 3 & $\left(\mathbf{e}_{2} \cdot \overline{\mathbf{e}}_{1}\right)\left(\mathbf{e}_{1} \times \overline{\mathbf{e}}_{2}\right) \cdot \hat{\mathbf{u}}$ \\
\hline 1 & 4 & $\left(\mathbf{e}_{1} \cdot \overline{\mathbf{e}}_{2}\right)\left(\mathbf{e}_{2} \times \overline{\mathbf{e}}_{1}\right) \cdot \hat{\mathbf{u}}$ \\
\hline 1 & 5 & $\left(\mathbf{e}_{2} \times \overline{\mathbf{e}}_{2}\right) \cdot \hat{\mathbf{u}}$ \\
\hline 1 & 6 & $\left(\mathbf{e}_{1} \cdot \mathbf{e}_{2}\right)\left(\overline{\mathbf{e}}_{1} \times \overline{\mathbf{e}}_{2}\right) \cdot \hat{\mathbf{u}}$ \\
\hline 2 & 1 & $\left(\mathbf{e}_{1} \cdot \mathbf{e}_{2}\right)\left(\hat{\mathbf{u}} \cdot \overline{\mathbf{e}}_{1}\right)\left(\hat{\mathbf{u}} \cdot \overline{\mathbf{e}}_{2}\right)-\left(\frac{1}{3}\right)\left(\mathbf{e}_{1} \cdot \mathbf{e}_{2}\right)\left(\overline{\mathbf{e}}_{1} \cdot \overline{\mathbf{e}}_{2}\right)$ \\
\hline 2 & 2 & $\left(\hat{\mathbf{u}} \cdot \mathbf{e}_{2}\right)\left(\hat{\mathbf{u}} \cdot \overline{\mathbf{e}}_{2}\right)-\frac{1}{3}$ \\
\hline 2 & 3 & $\left(\mathbf{e}_{1} \cdot \overline{\mathbf{e}}_{2}\right)\left(\hat{\mathbf{u}} \cdot \mathbf{e}_{2}\right)\left(\hat{\mathbf{u}} \cdot \overline{\mathbf{e}}_{1}\right)-\left(\frac{1}{3}\right)\left(\mathbf{e}_{1} \cdot \overline{\mathbf{e}}_{2}\right)\left(\mathbf{e}_{2} \cdot \overline{\mathbf{e}}_{1}\right)$ \\
\hline 2 & 4 & $\left(\mathbf{e}_{2} \cdot \overline{\mathbf{e}}_{1}\right)\left(\hat{\mathbf{u}} \cdot \mathbf{e}_{1}\right)\left(\hat{\mathbf{u}} \cdot \overline{\mathbf{e}}_{2}\right)-\left(\frac{1}{3}\right)\left(\mathbf{e}_{1} \cdot \overline{\mathbf{e}}_{2}\right)\left(\mathbf{e}_{2} \cdot \overline{\mathbf{e}}_{1}\right)$ \\
\hline 2 & 5 & $\left(\hat{\mathbf{u}} \cdot \mathbf{e}_{1}\right)\left(\hat{\mathbf{u}} \cdot \tilde{\mathbf{e}}_{1}\right)-\frac{1}{3}$ \\
\hline 2 & 6 & $\left(\overline{\mathbf{e}}_{1} \cdot \overline{\mathbf{e}}_{2}\right)\left(\hat{\mathbf{u}} \cdot \mathbf{e}_{1}\right)\left(\hat{\mathbf{u}} \cdot \mathbf{e}_{2}\right)-\left(\frac{1}{3}\right)\left(\mathbf{e}_{1} \cdot \mathbf{e}_{2}\right)\left(\overline{\mathbf{e}}_{1} \cdot \overline{\mathbf{e}}_{2}\right)$ \\
\hline 3 & 1 & $\begin{array}{l}\left(\mathbf{e}_{1} \times \overline{\mathbf{e}}_{1}\right) \cdot \hat{\mathbf{u}}\left(\hat{\mathbf{u}} \cdot \mathbf{e}_{2}\right)\left(\hat{\mathbf{u}} \cdot \overline{\mathbf{e}}_{2}\right)-\left(\frac{1}{5}\right)\left[\left(\mathbf{e}_{1} \times \overline{\mathbf{e}}_{1}\right) \cdot \mathbf{e}_{2}\left(\hat{\mathbf{u}} \cdot \overline{\mathbf{e}}_{2}\right)+\left(\mathbf{e}_{1} \times \overline{\mathbf{e}}_{1}\right) \cdot \overline{\mathbf{e}}_{2}\left(\hat{\mathbf{u}} \cdot \mathbf{e}_{2}\right)\right. \\
\left.\quad+\left(\mathbf{e}_{1} \times \overline{\mathbf{e}}_{1}\right) \cdot \hat{\mathbf{u}}\right]\end{array}$ \\
\hline 3 & 2 & $\begin{array}{l}\left(\mathbf{e}_{2} \times \overline{\mathbf{e}}_{1}\right) \cdot \hat{\mathbf{u}}\left(\hat{\mathbf{u}} \cdot \mathbf{e}_{1}\right)\left(\hat{\mathbf{u}} \cdot \overline{\mathbf{e}}_{2}\right)-\left(\frac{1}{5}\right)\left[\left(\mathbf{e}_{2} \times \overline{\mathbf{e}}_{1}\right) \cdot \mathbf{e}_{1}\left(\hat{\mathbf{u}} \cdot \overline{\mathbf{e}}_{2}\right)+\left(\mathbf{e}_{2} \times \overline{\mathbf{e}}_{1}\right) \cdot \overline{\mathbf{e}}_{2}\left(\hat{\mathbf{u}} \cdot \mathbf{e}_{1}\right)\right. \\
\left.\quad+\left(\mathbf{e}_{2} \times \overline{\mathbf{e}}_{1}\right) \cdot \hat{\mathbf{u}}\left(\mathbf{e}_{1} \cdot \overline{\mathbf{e}}_{2}\right)\right]\end{array}$ \\
\hline 3 & 3 & $\begin{array}{l}\left(\overline{\mathbf{e}}_{1} \times \overline{\mathbf{e}}_{2}\right) \cdot \hat{\mathbf{u}}\left(\hat{\mathbf{u}} \cdot \mathbf{e}_{1}\right)\left(\hat{\mathbf{u}} \cdot \mathbf{e}_{2}\right)-\left(\frac{1}{5}\right)\left[\left(\overline{\mathbf{e}}_{1} \times \overline{\mathbf{e}}_{2}\right) \cdot \mathbf{e}_{1}\left(\hat{\mathbf{u}} \cdot \mathbf{e}_{2}\right)+\left(\overline{\mathbf{e}}_{1} \times \overline{\mathbf{e}}_{2}\right) \cdot \mathbf{e}_{2}\left(\hat{\mathbf{u}} \cdot \mathbf{e}_{1}\right)\right. \\
\left.\quad+\left(\overline{\mathbf{e}}_{1} \times \overline{\mathbf{e}}_{2}\right) \cdot \hat{\mathbf{u}}\left(\mathbf{e}_{1} \cdot \mathbf{e}_{2}\right)\right]\end{array}$ \\
\hline 4 & 1 & $\begin{array}{l}\left(\hat{\mathbf{u}} \cdot \mathbf{e}_{1}\right)\left(\hat{\mathbf{u}} \cdot \mathbf{e}_{2}\right)\left(\hat{\mathbf{u}} \cdot \overline{\mathbf{e}}_{1}\right)\left(\hat{\mathbf{u}} \cdot \overline{\mathbf{e}}_{2}\right)-\left(\frac{1}{7}\right)\left[\left(\mathbf{e}_{1} \cdot \mathbf{e}_{2}\right)\left(\hat{\mathbf{u}} \cdot \overline{\mathbf{e}}_{1}\right)\left(\hat{\mathbf{u}} \cdot \overline{\mathbf{e}}_{2}\right)+\left(\hat{\mathbf{u}} \cdot \mathbf{e}_{2}\right)\left(\hat{\mathbf{u}} \cdot \overline{\mathbf{e}}_{2}\right)\right. \\
\quad+\left(\mathbf{e}_{1} \cdot \overline{\mathbf{e}}_{2}\right)\left(\hat{\mathbf{u}} \cdot \overline{\mathbf{e}}_{1}\right)\left(\hat{\mathbf{u}} \cdot \mathbf{e}_{2}\right)+\left(\mathbf{e}_{2} \cdot \overline{\mathbf{e}}_{1}\right)\left(\hat{\mathbf{u}} \cdot \mathbf{e}_{1}\right)\left(\hat{\mathbf{u}} \cdot \overline{\mathbf{e}}_{2}\right)+\left(\hat{\mathbf{u}} \cdot \mathbf{e}_{1}\right)\left(\hat{\mathbf{u}} \cdot \overline{\mathbf{e}}_{1}\right) \\
\left.\quad+\left(\overline{\mathbf{e}}_{1} \cdot \overline{\mathbf{e}}_{2}\right)\left(\hat{\mathbf{u}} \cdot \mathbf{e}_{1}\right)\left(\hat{\mathbf{u}} \cdot \mathbf{e}_{2}\right)\right]+\left(\frac{1}{35}\right)\left[\left(\mathbf{e}_{1} \cdot \mathbf{e}_{2}\right)\left(\overline{\mathbf{e}}_{1} \cdot \overline{\mathbf{e}}_{2}\right)+1+\left(\mathbf{e}_{1} \cdot \overline{\mathbf{e}}_{2}\right)\left(\mathbf{e}_{2} \cdot \overline{\mathbf{e}}_{1}\right)\right]\end{array}$ \\
\hline
\end{tabular}


given by $\mathbf{s}=\left(\mathbf{k}_{1}+\mathbf{k}_{2}\right)$; the resultant rate equation may be expressed as

$$
\begin{aligned}
\left\langle\Gamma_{2 d}\right\rangle= & \left(\frac{2 \pi \rho_{f}}{h}\right) K^{2}\left\{\sum_{p, q} g_{(4 ; 0)}^{p q} A^{(0 ; p)}(\hat{\mathbf{s}} ;\right. \\
& \times T_{d}^{(0 ; q)}\left(\alpha, \alpha, \alpha, \alpha ; A_{1}, A_{2}, A_{1} A_{2} ; \omega_{\alpha 0}, \omega_{\alpha 0}\right) \\
& +\sum_{j=0} \sum_{p, q} \frac{(2 j) !}{2^{j}(j !)^{2}} i^{j} j_{j}(|\hat{\mathbf{s}}| R) g_{(4, j)}^{p q} A^{(j ; p)}(\hat{\mathbf{s}}) \\
& \left.\times T_{d}^{(j ; q)}\left(\alpha, \alpha, \alpha, \alpha ; A_{1}, A_{2}, A_{2}, A_{1} ; \omega_{\alpha 0},-\omega_{\alpha 0}\right)\right\} \\
& +\left\{A_{1}, V \leftrightarrow A_{2}, \bar{V}\right\} .
\end{aligned}
$$

Once again, the above result may be adapted to obtain the fully averaged rate by replacing each $T_{d}(j ; q)$ by its fully averagged counterpart $\ll T_{d}^{(j ; a)} 》$.

\section{RANGE-DEPENDENCE}

The results of Sections VI and VII are applicable: over an unrestricted range of separations between the two participating centers, and it should be recalled that the quantum electrodynamical formalism ernployed for their derivation automatically incorporates relativistic retardation effects. It is, therefore, instructive to examine the detailed dependence: of the rate equations on intermolecular separation. The study of the lim ting short- and long-range behavior of the rates is facilitated through the identification of two regions known respectively as the near-zone and the far-zone, in each of which wellcharacterized asymptotic behavior can be identified. The limiting ranges of these zones is determined, for each process, by the character of the complex retarded resonance electric dipole-electric dipole interaction tensor $V_{k l}(\omega, \mathbf{R})$, and also the nature of any exponential terms appearing in the pre-averaged rate equation.

The general form of the interaction tensor $V_{k l}(\omega, \mathbf{R})$, applicable for the entire range of pair separations, is given by Eq. (5.12). The appropriate near-zone form of the interaction can be idenlified with the static (zerofrequency) result, $V_{k l}(0, \mathbf{R})$, obtained in the limit where $\omega R / c \ll 1$, and is given by:

$$
\lim _{\omega R^{\prime} / c<1} V_{k l}(\omega, \mathbf{R})=V_{k l}(0, \mathbf{R})=\frac{1}{4 \pi \varepsilon_{0} R^{3}}\left(\delta_{k l}-3 \hat{R}_{k} \hat{R}_{l}\right)
$$

which is traceless. The far-zone form of the interaction tensor is taken where 
$\omega R / c \gg 1$, and thus long-range behavior is determined by the limit

$$
\lim _{\omega R / c \gg 1} V_{k i t}(\omega, \mathbf{R})=\frac{\omega^{2}}{4 \pi c^{2} \varepsilon_{0} R}\left(\hat{R}_{k} \hat{R}_{l}-\delta_{k l}\right) e^{i \omega R / c}
$$

which is purely transverse with respect to the intermolecular vector $\mathbf{R}$. Since the frequency parametrization of the interaction tensor is different for each of the four cases of synergistic two-photon absorption under consideration, the conditions under which the limiting near- or far-zone behavior ensues also differ markedly.

The exponential phase factors which appear in the pre-averaged rate equations (6.7), (6.9), and (6.12), i.e., all cases except that of single-beam cooperative absorption, Eq. (6.3), introduce further considerations. Here, what might be termed near-zone behavior is obtained when these exponentials can be approximated by unity. In the corresponding rotationally averaged results of Section VII, the corresponding level of approximation results in retention of just the leading terms involving the $j_{0}$ spherical Bessel functions, since each $j_{j}(\alpha)$ falls off as $\alpha^{-j}$ in the near-zone. Consequently only the $j=0$ terms contribute appreciably to the sums over $j$ in the second terms of Eqs. (7.8), (7.10), and (7.12), thereby significantly reducing the complexity of the results. Overall, the near-zone and far-zone limits to the rate equations are therefore subject to conditions imposed both by the nature and form of interaction tensor and also of any phase factors which may be present for each case. A complete breakdown of the boundary conditions for the four processes described in this review is given explicitly in Table VI.

Having defined the short- and long-range limits, it is now possible to examine the general behavior of the rate equations for synergistic photoabsorption within these regions. In the near-zone, since the limiting (static) form of the complex retarded resonance electric dipole-electric dipole

TABLE VI

Near- and Far-Zone Boundary Conditions Resulting from the Form of the Phase Factors and Parametrization of the Interaction Tensor

\begin{tabular}{lll}
\hline Two-photon absorption process & Near-zone & Far-zone \\
\hline Single-beam cooperative & $\left|\omega_{\alpha 0}-\omega\right| R / c \ll 1$ & $\left|\omega_{\alpha 0}-\omega\right| R / c \gg 1$ \\
Single-beam distributive & $2 k R \ll 1$ & $\left(\omega_{\alpha 0} R / c, \omega_{\beta 0} R / c\right) \gg 1$ \\
& $\Rightarrow\left(\omega_{\alpha 0} R / c, \omega_{\beta 0} R / c\right) \ll 1$ & $\Rightarrow 2 k R \gg 1$ \\
Double-beam cooperative & $\left|\omega_{\alpha 0}-\omega_{1}\right| R / c \ll 1$ & $\left|\omega_{\alpha 0}-\omega_{1}\right| R / c \gg 1$ \\
& $\left|\mathbf{k}_{1}-\mathbf{k}_{2}\right| R \ll 1$ & $\left|\mathbf{k}_{1}-\mathbf{k}_{2}\right| R \gg 1$ \\
Double-beam distributive & $\left|\mathbf{k}_{1}+\mathbf{k}_{2}\right| R \ll 1$ & $\omega_{\alpha 0} R / c \gg 1$ \\
& $\omega_{\alpha 0} R / c \ll 1$ & $\left|\mathbf{k}_{1}+\mathbf{k}_{2}\right| R \gg 1$ \\
\hline
\end{tabular}


interaction (Eq. 8.1) has an $R^{-3}$ dependence, the corresponding near-zone rate expressions with quadratic dependence on $V$ vary as $R^{-6}$. This kind of distance dependence is well known in the Förster theory of dipolar resonance energy transfer (Förster 1949). Thus, the rate of timolecular mean-frequency absorption involving pairs of free molecules falls off very rapidly within the near-zone.

For the far-zone case where molecules are separated by comparatively large distances, the rate equations for the three cases with radiative phase factors become complicated since all the spherical Bessel functions make comparable contributions to the result. Here an iriverse-square variation with molecular separation results from the quadratic dependence on the longrange limit of $V$, expressed by Eq. (8.2). It is therefore worth noting that although the rate of synergistic absorption continues to fall off as the separation between interacting molecules increases, it does not fall off anywhere near as quickly as might be expected rom an examination of the near-zone behavior. While inverse-square rate dependences of this type are rare and therefore often suspect in atomic and molecular physics, the far-zone behavior reflects an exact asymptotic equivalence to the classical results for radiative energy transfer; a detailed proof has been given by Andrews and Hopkins (1988b). This comparison establishes the correctness of the longrange distance dependence of the virtual photor coupling.

It is interesting to note that it is not essential for both centers involved in a distributive absorption process to be simultaneously located at the focus of the two laser beams. Naturally, one of the two absorbing centers must be irradiated by both beams, but the second center alssorbs only a virtual photon and need not, therefore, be within the volume of sample irradiated by either beam. This has an unusual consequence for the case where the two centers are discrete molecules. The number of potential partners, $A_{2}$, which may be involved in the distributive excitation of any particular molecule $A_{1}$ greatly exceeds the number available for cooperative excitation, and, assuming a uniform sample density, increases with the square of the intermolecular distance. Since the long-range form of the rate equation has an inverse-square dependence on the separation, the total of all the contributions from partner molecules within a shell of given thickness centered on $A_{1}$ is, in the long-range limit, independent of the shell radius. This surprising result is a molecular analogue of the astrophysical problem known as Olber's Paradox, which poses the question of why the sky is not uniformly bright with starlight. This paradox arises in a similar way since, although starlight intensity drops off with the square of distance, the number of stars in a homogeneous universe also increases quadratically with distance from any given reference point. In both cases, the resolution of the paradox is connected with a consideration of the neglected effects of light scattering. 
One of the main differences between the cooperative and distributive mechanisms lies in the range over which the limiting near-zone behavior occurs. In the cooperative cases, the near-zone form of the interaction tensor applies where $R \Delta \omega / c \ll 1, \Delta \omega$ representing the energy mismatch propagated by the virtual photon (see Table VI). The supplementary condition $\left|\mathbf{k}_{1}-\mathbf{k}_{2}\right| R \ll 1$ in the double-beam case is less restrictive, except in an

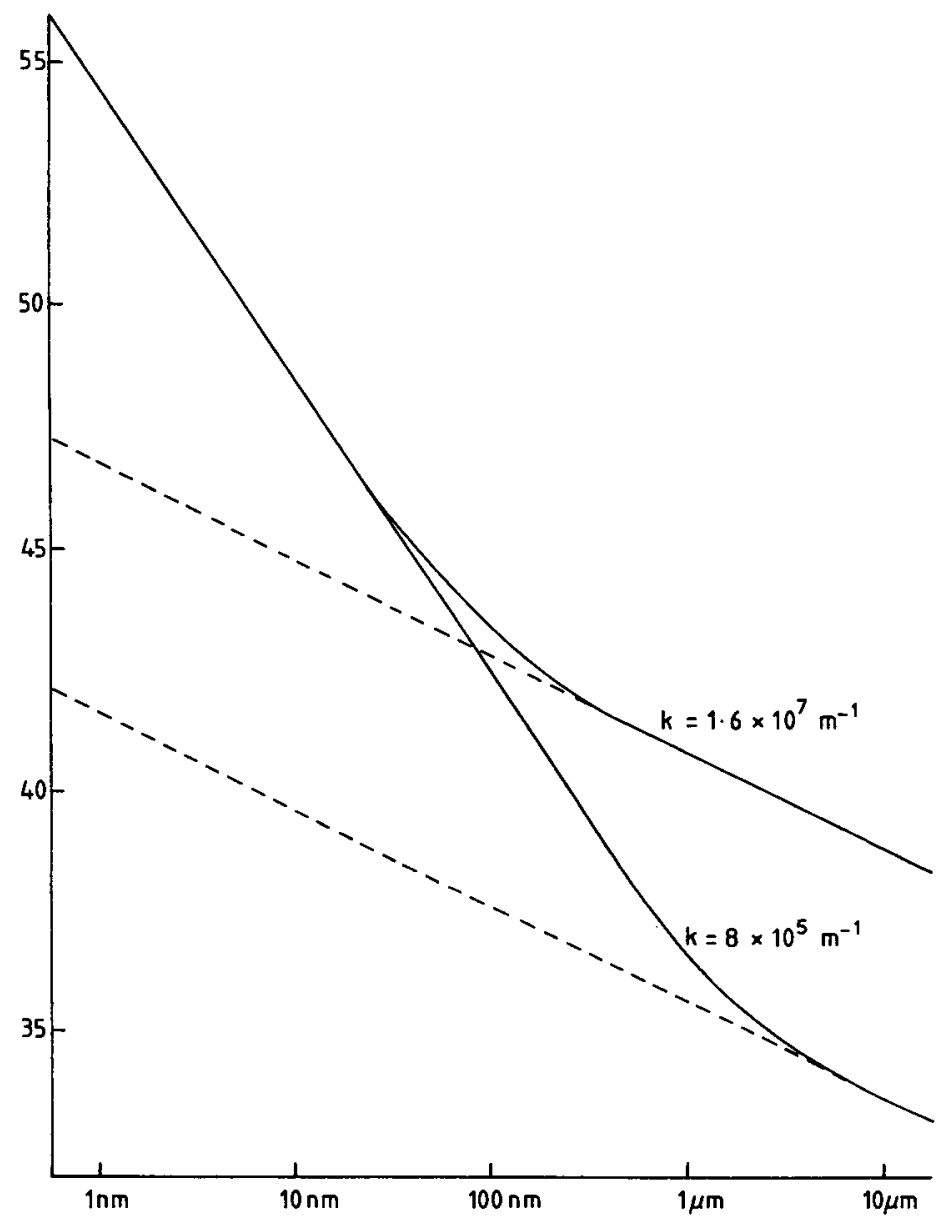

Figure 9. Logarithmic plots on an arbitrary vertical scale of the function $V_{i j}(c k, \mathbf{R}) \bar{V}_{i j}(c k, \mathbf{R})$ (solid lines) which describes the dependence on intermolecular distance $R$ of synergistic photoabsorption in free molecules. The values of $k$ for the upper and lower curves are typical of the distributive and cooperative mechanisms, respectively. The broken lines show the asymptotic $k^{4} R^{-2}$ behavior, whose relative displacement is $5.20\left(=\log (20)^{4}\right)$. 
experimentally unusual configuration where the two photons are absorbed from essentially counterpropagating beams. However, in the distributive cases, the most severe constraints for near-zone behavior are imposed by the condition $|\mathbf{s}| R \ll 1$, where $\mathbf{s}$ is the wave-vector sum for the two absorbed photons. This automatically guarantees satisfaction of the condition $\omega_{\alpha 0} R / c \ll 1$, again except in counterpropagating; beam configurations.

In certain cases, significant differences in the extent of the near-zone result from this distinction, especially when the excitec-state energies are large but similar. Figure 9 illustrates this point with a $\log -\log$ plot of the general function $V_{i j}(c k, \mathbf{R}) \bar{V}_{i j}(c k, \mathbf{R})$ which occurs in all triply averaged rate equations. The upper curve is plotted for a value of $k=1.6 \times 10^{7} \mathrm{~m}^{-1}$, corresponding to distributive conveyance of an electronic energy $E_{\beta 0}$ with a wavelength of about $400 \mathrm{~nm}$. The lower curve with $k=8 \times 10^{5} \mathrm{~m}^{-1}$ corresponds to the cooperative mechanism where only an electronic energy difference (nominally $E_{\beta 0} / 20$ ) is conveyed; here the difference equates to a vibrational energy with a wavenumber of around $1250 \mathrm{~cm}^{-1}$. At short clistances the two graphs are indistinguishable and display the near-zone $R^{-6}$ dependence. However the extent of the near-zone for the former case is much shorter, with the limiting far-zone $R^{-2}$ behavior already established at $K=1 \mu \mathrm{m}$; for the latter case, far-zone behavior obtains at $R=10 \mu \mathrm{m}$. The result of this difference is that the long-range rates (which vary with $k^{4}$ ) differ by a factor of $(20)^{4}=160,000$ in favor of the distributive mechanism (Andrews 1989).

An even more striking illustration concerns the classic case of synergistic $2{ }_{0}^{1} 4_{0}^{3}$ transitions in a mixture of formaldehyde and deuterioformaldehyde, where the absorption wavenumbers are $30,240.15$ and $30,147.62 \mathrm{~cm}^{-1}$, respectively (Moule and Walsh 1975). Here the near-zone for single-beam two-photon absorption extends to values of $R$ up to $\sim 10 \mu \mathrm{m}$ : however for the distributive mechanism, near-zone behavior extends only to $\sim 100 \mathrm{~nm}$, and the far-zone $R^{-2}$ behavior is already dominant at $10 \mu \mathrm{m}$. Here the distributive mechanism is favored by a factor of $\sim 6 \times 10^{8}$.

\section{POLARIZATION DEPENDENCE}

In the experimental studies that have identilied synergistic two-photon processes, little attention has yet been paid to effects of laser polarization. However, judicious control of polarization stould enable more detailed information to be derived from the recorded spectra, particularly in the case of two-beam excitation where more polarization parameters are variable. This section provides a detailed theory of the polarization dependence of synergistic two-photon absorption in fluid meclia, including an analysis of some unusual dichroic effects. 


\section{A. Single-Beam Polarization Parameters}

In terms of polarization analysis, the single-beam cases yield little of interest, since only one independently variable polarization parameter, $\zeta$, arises. This is defined by the relation

$$
\zeta=(\mathbf{e} \times \overline{\mathbf{e}}) \cdot \hat{\mathbf{k}}
$$

Although rate equations have been cast in terms of both $\zeta$ and a second parameter, $\eta$, defined by Eq. (7.4),

$$
\eta=(\mathbf{e} \cdot \mathbf{e})(\overline{\mathbf{e}} \cdot \overline{\mathbf{e}})
$$

it can in fact be shown that the two are related through the equation

$$
\eta=1+\zeta^{2}
$$

Both parameters distinguish the degree of helicity of the incident radiation. For instance, $\eta$ assumes the value of unity for plane polarized light and zero for circularly polarized light, and may therefore be regarded as a direct measure of the degree of ellipticity of the laser beam. However, only $\zeta$, which is zero for plane polarized light, differentiates the sense of handedness in circular or elliptical polarizations, and for the circularly polarized cases we have

$$
\zeta^{(L)}=-i \text { and } \zeta^{(R)}=i
$$

On casting the rate equations entirely in terms of $\zeta$, it becomes evident that the result for the single-beam cooperative case contains only terms in $\zeta^{2}$ and numerical terms, while additional terms linear in $\zeta$ occur in the single-beam distributive case. Hence, the odd- $j$ terms in Eq. (7.8) only contribute to the result when circularly or elliptically polarized incident radiation is employed, and their sign is then dependent on the handedness of that incident radiation. A direct consequence of this is the exhibition of two-photon circular dichroism in the distributive absorption process for pairs of molecules with fixed mutual orientations: no such effect can occur under the cooperative mechanism.

\section{B. Two-Photon Circular Dichroism}

Two-photon circular dichroism (Meath and Power 1987) was first predicted over a decade ago (Tinoco 1975; Power 1975; Andrews 1976), and is closely related to two-photon resonance effects in optical rotatory dispersion, the 
first observations of which have recently been made by Gedanken and Tamir (1988). The extent of circular dichroism in distributive absorption can be expressed through the ratio of the difference in rates for left- and right-circular polarization and the mean rate, i.e.,

$$
\Delta^{(L / R)}=\frac{\Gamma^{(L)}-\Gamma^{(R)}}{\frac{1}{2}\left(\Gamma^{(L)}+\Gamma^{(R)}\right)}
$$

which, using Eqs. (7.8) and (9.4), gives

$$
\Delta^{(L / R)}=\frac{c j_{1}(2 k R)+e j_{3}(2 k: R)}{a+b j_{0}(2 k R)+d j_{2}(2 k R)+f j_{4}(2 k R)}
$$

where the explicit expressions for the coefficients of the spherical Bessel functions are as follows:

$$
\begin{aligned}
a= & 56\left(3 \chi_{(i j) k}^{\alpha 0} \bar{\chi}_{(i j) o}^{\alpha 0}-\chi_{(i i) k}^{\alpha 0} \bar{\chi}_{(j j) o}^{\alpha 0}\right) \mu_{l}^{\beta 0} \bar{\mu}_{p}^{\beta 0} V_{k l}\left(\omega_{\alpha 0}, \mathbf{R}\right) \bar{V}_{o p}\left(\omega_{\alpha 0}, \mathbf{R}\right) \\
& +\left(\alpha, \omega_{\alpha 0} \leftrightarrow \beta, \omega_{\beta 0}\right) \\
b= & 56\left(3 \chi_{(i j) k}^{\beta 0} \bar{\chi}_{(i j) o}^{\alpha 0}-\chi_{(i i) k}^{\beta 0} \bar{\chi}_{(j j) o}^{\alpha 0}\right) \mu_{l}^{\alpha 0} \bar{\mu}_{p}^{\beta 0} V_{k l}\left(\omega_{\beta 0}, \mathbf{R}\right) \bar{V}_{o p}\left(\omega_{\alpha 0}, \mathbf{R}\right) \\
& +\left(\alpha, \omega_{\alpha 0} \leftrightarrow \beta, \omega_{\beta 0}\right) \\
c= & 672 \varepsilon_{i m t} \hat{R}_{t} \chi_{(i j) k}^{\beta 0} \bar{\chi}_{(j m) o}^{\alpha 0} \mu_{l}^{\alpha 0} \bar{\mu}_{p}^{\beta 0} V_{k l}\left(\omega_{\beta 0}, \mathbf{R}\right) \bar{V}_{o p}\left(\omega_{x 0}, \mathbf{R}\right) \\
& +\left(\alpha, \omega_{\alpha 0} \leftrightarrow \beta, \omega_{\beta 0}\right) \\
d= & 20\left(8 \chi_{(i i) k}^{\beta 0} \bar{\chi}_{(j j) o}^{\alpha 0}-12 \chi_{(i j) k}^{\beta 0} \bar{\chi}_{(i j) o}^{\alpha 0}-12 \chi_{(i j) k}^{\beta 0} \bar{\chi}_{(j j) o}^{\alpha 0} \hat{R}_{i} \hat{R}_{j}-12 \chi_{(i i) k}^{\beta 0} \bar{\chi}_{(m n) o}^{\alpha 0} \hat{R}_{m} \hat{R}_{n}\right. \\
& \left.+36 \chi_{(i j) k}^{\beta 0} \bar{\chi}_{(j m) o}^{\alpha 0} \hat{R}_{i} \hat{R}_{m}\right) \mu_{l}^{\alpha 0} \bar{\mu}_{p}^{\beta 0} V_{k l}\left(\omega_{\beta 0}, \mathbf{R}\right) \bar{V}_{o p}\left(\omega_{\alpha 0}, \mathbf{R}\right) \\
& +\left(\alpha, \omega_{\alpha 0} \leftrightarrow \beta, \omega_{\beta 0}\right), \\
e= & 168 \varepsilon_{i m t} \hat{R}_{t}\left(5 \chi_{(i j) k}^{\beta 0} \bar{\chi}_{(m n) o}^{\alpha 0} \hat{R}_{j} \hat{R}_{n}-\chi_{(i j) k}^{\beta 0} \bar{\chi}_{(j m) o}^{\alpha 0}\right) \mu_{l}^{\alpha 0} \bar{\mu}_{p}^{\beta 0} V_{k l}\left(\omega_{\beta 0}, \mathbf{R}\right) \bar{V}_{o p}\left(\omega_{\alpha 0}, \mathbf{R}\right) \\
& +\left(\alpha, \omega_{\alpha 0} \leftrightarrow \beta, \omega_{\beta 0}\right), \\
f= & 6\left(35 \chi_{(i j) k}^{\beta 0} \bar{\chi}_{(m n) o}^{\alpha 0} \hat{R}_{i} \hat{R}_{j} \hat{R}_{m} \hat{R}_{n}-5 \chi_{(i i) k}^{\beta 0} \bar{\chi}_{(m n) o}^{\alpha 0} \hat{R}_{m} \hat{R}_{n}-5 \chi_{(i j) k}^{\beta 0} \bar{\chi}_{(m m) o}^{\alpha 0} \hat{R}_{i} \hat{R}_{j}\right. \\
& -20 \chi_{(i j) k}^{\beta 0} \bar{\chi}_{(i n) o}^{\alpha 0} \hat{R}_{j} \hat{R}_{n}+\chi_{(i i) k}^{\beta 0} \bar{\chi}_{(j j) o}^{\alpha 0} \\
& \left.+2 \chi_{(i j) k}^{\beta 0} \bar{\chi}_{(i j) o}^{\alpha 0}\right) \mu_{l}^{\alpha 0} \bar{\mu}_{p}^{\beta 0} V_{k l}\left(\omega_{\beta 0}, \mathbf{R}\right) \bar{V}_{o p}\left(\omega_{\alpha 0}, \mathbf{R}\right) \\
& +\left(\alpha, \omega_{\alpha 0} \leftrightarrow \beta, \omega_{\beta 0}\right) .
\end{aligned}
$$


The result given by Eq. (9.6) is applicable to a rotating pair with arbitrary separation of the centers A and B. However, in such a case where the intermolecular distance $R$ is fixed, it is generally appropriate to take the near-zone limit, based on the arguments discussed in the last section. By taking the dominant terms in the numerator and denominator of Eq. (9.6), we thus obtain the simpler result that

where

$$
\Delta^{(L / R)} \simeq(x / y) k R,
$$

$$
\begin{aligned}
x= & 448 \varepsilon_{i m t} \hat{R}_{t}\left[\left\{\chi_{(i j) k}^{\beta 0} \bar{\chi}_{(m j) o}^{\alpha 0} \mu_{k}^{\alpha 0} \bar{\mu}_{o}^{\beta 0}-3 \chi_{(i j) k}^{\beta 0} \bar{\chi}_{(m j) o}^{\alpha 0} \mu_{k}^{\alpha 0} \bar{\mu}_{p}^{\beta 0} \hat{R}_{o} \hat{R}_{p}\right.\right. \\
& \left.-3 \chi_{(i j) k}^{\beta 0} \bar{\chi}_{(m j) o}^{\alpha 0} \mu_{l}^{\alpha 0} \bar{\mu}_{o}^{\beta 0} \hat{R}_{k} \hat{R}_{l}+9 \chi_{(i j) k}^{\beta 0} \bar{\chi}_{(m j) o}^{\alpha 0} \mu_{l}^{\alpha 0} \bar{\mu}_{p}^{\beta 0} \hat{R}_{k} \hat{R}_{l} \hat{R}_{o} \hat{R}_{p}\right\} \\
& +\{\alpha \leftrightarrow \beta\}], \\
y= & 56\left[\left\{-\chi_{(i i) k}^{\alpha 0} \bar{\chi}_{(m m) o}^{\alpha 0} \mu_{k}^{\beta 0} \bar{\mu}_{o}^{\beta 0}+3 \chi_{(i i) k}^{\alpha 0} \bar{\chi}_{(m m) o}^{\alpha 0} \mu_{k}^{\beta 0} \bar{\mu}_{p}^{\beta 0} \hat{R}_{o} \hat{R}_{p}\right.\right. \\
& +3 \chi_{(i i) k}^{\alpha 0} \bar{\chi}_{(m m) o}^{\alpha 0} \mu_{l}^{\beta 0} \bar{\mu}_{o}^{\beta 0} \hat{R}_{k} \hat{R}_{l}-9 \chi_{(i i) k}^{\alpha 0} \bar{\chi}_{(m m) o}^{\alpha 0} \mu_{l}^{\beta 0} \bar{\mu}_{p}^{\beta 0} \hat{R}_{k} \hat{R}_{l} \hat{R}_{o} \hat{R}_{p} \\
& +3 \chi_{(i j) k}^{\alpha 0} \bar{\chi}_{(i j) o}^{\alpha 0} \mu_{k}^{\beta 0} \bar{\mu}_{o}^{\beta 0}-9 \chi_{(i j) k}^{\alpha 0} \bar{\chi}_{(i j) o}^{\alpha 0} \mu_{k}^{\beta 0} \bar{\mu}_{p}^{\beta 0} \hat{R}_{o} \hat{R}_{p} \\
& -9 \chi_{(i j) k}^{\alpha 0} \bar{\chi}_{(i j) o}^{\alpha 0} \mu_{l}^{\beta 0} \bar{\mu}_{o}^{\beta 0} \hat{R}_{k} \hat{R}_{l}+27 \chi_{(i j) k}^{\alpha 0} \bar{\chi}_{(i j) o}^{\alpha 0} \mu_{l}^{\beta 0} \bar{\mu}_{p}^{\beta 0} \hat{R}_{k} \hat{R}_{l} \hat{R}_{o} \hat{R}_{p} \\
& -\chi_{(i i) k}^{\beta 0} \bar{\chi}_{(m m) o}^{\alpha 0} \mu_{k}^{\alpha 0} \bar{\mu}_{o}^{\beta 0}+3 \chi_{(i i) k}^{\beta 0} \bar{\chi}_{(m m) o}^{\alpha 0} \mu_{k}^{\alpha 0} \bar{\mu}_{p}^{\beta 0} \hat{R}_{o} \hat{R}_{p} \\
& +3 \chi_{(i i) k}^{\beta 0} \bar{\chi}_{(m m) o}^{\alpha 0} \mu_{l}^{\alpha 0} \bar{\mu}_{o}^{\beta 0} \hat{R}_{k} \hat{R}_{l}-9 \chi_{(i i) k}^{\beta 0} \bar{\chi}_{(m m) o}^{\alpha 0} \mu_{l}^{\alpha 0} \bar{\mu}_{p}^{\beta 0} \hat{R}_{k} \hat{R}_{l} \hat{R}_{o} \hat{R}_{p} \\
& +3 \chi_{(i j) k}^{\beta 0} \bar{\chi}_{(i j) o}^{\alpha 0} \mu_{k}^{\alpha 0} \bar{\mu}_{o}^{\beta 0}-9 \chi_{(i j) k}^{\beta 0} \bar{\chi}_{(i j) o}^{\alpha 0} \mu_{k}^{\alpha 0} \bar{\mu}_{p}^{\beta 0} \hat{R}_{o} \hat{R}_{p} \\
& \left.-9 \chi_{(i j) k}^{\beta 0} \bar{\chi}_{(i j) o}^{\alpha 0} \mu_{l}^{\alpha 0} \bar{\mu}_{o}^{\beta 0} \hat{R}_{k} \hat{R}_{l}+27 \chi_{(i j) k}^{\beta 0} \bar{\chi}_{(i j) o}^{\alpha 0} \mu_{l}^{\alpha 0} \bar{\mu}_{p}^{\beta 0} \hat{R}_{k} \hat{R}_{l} \hat{R}_{o} \hat{R}_{p}\right\} \\
& +\{\alpha \leftrightarrow \beta\}]
\end{aligned}
$$

Systems involving two centers with fixed mutual orientation have been shown to exhibit other circular differential effects with a similar linear dependence on the separation. Examples include circular differential Rayleigh and Raman scattering (Barron and Buckingham 1974; Andrews and Thirunamachandran 1978), optical rotation (Barron 1975) and twophoton circular dichroism in which only one chromophore is excited (Andrews 1976). As might be expected, on performing subsequent rotational averages for the case where A and B are randomly oriented, the odd- $j$ terms in the rate equations vanish and consequently no circular dichroism is displayed.

\section{Double-Beam Polarization Studies}

In terms of polarization analysis, double-beam cooperative and doublebeam distributive two-photon absorption display identical behavior since 
essentially the same set of polarization parameters, $A^{(j ; p)}$, arises in each. The only difference is that the role of the wave-vector difference $\mathbf{u}$ in the cooperative case is played by the wave-vector sum $\mathbf{s}$ in the distributive case. For any particular laser beam and polarization geometry, the values of the nineteen $A^{(j ; p)}$ which arise can be directly calculated and inserted into the relevant rate equations to give the corresponding rates. Consideration is given here to a number of polarization combinations in which the two laser beams are co-propagating. This represents the experimentally most useful configuration since it maximizes the volume of sample traversed by both beams.

The values of $A^{(j ; p)}$ for seven experimentally useful combinations of plane and circular polarizations are listed in Table VII. Where both laser beams are plane polarized, the values of the polarization parameters are given for the cases where the polarization planes of the two beams are mutually parallel (column 1) or perpendicular (column 2). The first point to notice is that in

TABLE VII

Values of the Polarization Parameters $A^{(j ; p)}(\hat{\mathbf{u}})$ for Seven Polarization Combinations with Co-propagating Beams

\begin{tabular}{ccccccccc}
\hline$j$ & $p$ & $e_{1}^{\mathbf{P}} \| e_{2}^{\mathbf{P}}$ & $e_{1}^{\mathbf{P}} \perp e_{2}^{\mathbf{P}}$ & $e_{1}^{\mathbf{P}} ; e_{2}^{\mathbf{L}}$ & $e_{1}^{\mathbf{P}} ; e_{2}^{\mathbf{R}}$ & $e_{1}^{\mathbf{L}} ; e_{2}^{\mathbf{L}}$ & $e_{1}^{\mathbf{R}} ; e_{2}^{\mathbf{R}}$ & $e_{1}^{\mathbf{L}} ; e_{2}^{\mathbf{R}}$ \\
\hline 0 & 1 & 1 & 0 & $\frac{1}{2}$ & $\frac{1}{2}$ & 0 & 0 & 1 \\
0 & 2 & 1 & 1 & 1 & 1 & 1 & 1 & 1 \\
0 & 3 & 1 & 0 & $\frac{1}{2}$ & $\frac{1}{2}$ & 1 & 1 & 0 \\
1 & 1 & 0 & 0 & $i / 2$ & $-i / 2$ & 0 & 0 & $-i$ \\
1 & 2 & 0 & 0 & 0 & 0 & $-i$ & $i$ & $-i$ \\
1 & 3 & 0 & 0 & $-i / 2$ & $i / 2$ & $-i$ & $i$ & 0 \\
1 & 4 & 0 & 0 & $-i / 2$ & $i / 2$ & $-i$ & $i$ & 0 \\
1 & 5 & 0 & 0 & $-i$ & $i$ & $-i$ & $i$ & $i$ \\
1 & 6 & 0 & 0 & $-i / 2$ & $i / 2$ & 0 & 0 & $i$ \\
2 & 1 & $-\frac{1}{3}$ & 0 & $-\frac{1}{6}$ & $-\frac{1}{6}$ & 0 & 0 & $-\frac{1}{3}$ \\
2 & 2 & $-\frac{1}{3}$ & $-\frac{1}{3}$ & $-\frac{1}{3}$ & $-\frac{1}{3}$ & $-\frac{1}{3}$ & $-\frac{1}{3}$ & $-\frac{1}{3}$ \\
2 & 3 & $-\frac{1}{3}$ & 0 & $-\frac{1}{6}$ & $-\frac{1}{6}$ & $-\frac{1}{3}$ & $-\frac{1}{3}$ & 0 \\
2 & 4 & $-\frac{1}{3}$ & 0 & $-\frac{1}{6}$ & $-\frac{1}{6}$ & $-\frac{1}{3}$ & $-\frac{1}{3}$ & 0 \\
2 & 5 & $-\frac{1}{3}$ & $-\frac{1}{3}$ & $-\frac{1}{3}$ & $-\frac{1}{3}$ & $-\frac{1}{3}$ & $-\frac{1}{3}$ & $-\frac{1}{3}$ \\
2 & 6 & $-\frac{1}{3}$ & 0 & $-\frac{1}{6}$ & $-\frac{1}{6}$ & 0 & 0 & $-\frac{1}{3}$ \\
3 & 1 & 0 & 0 & 0 & 0 & $i / 5$ & $-i / 5$ & $-i / 5$ \\
3 & 2 & 0 & 0 & $-i / 2$ & $i / 2$ & $i / 5$ & $-i / 5$ & 0 \\
3 & 3 & 0 & 0 & $-i / 2$ & $i / 2$ & 0 & 0 & $i / 5$ \\
4 & 1 & $\frac{3}{35}$ & $\frac{1}{35}$ & $\frac{2}{35}$ & $\frac{2}{35}$ & $\frac{2}{35}$ & $\frac{2}{35}$ & $\frac{2}{35}$ \\
\hline
\end{tabular}


both cases the odd $-j$ parameters vanish, so that the leading $j_{0}$ terms represent a particularly good approximation in the near-zone. Secondly, the difference in the values of $\boldsymbol{A}^{(j ; p)}$ for the two cases indicates a linear dichroism which is a known characteristic of two-photon absorption (Monson and McClain 1970).

\section{Natural Two-Photon Circular Dichroism}

In each of the remaining polarization conditions, there are generally contributions from all values of $j$, and the rate expressions are accordingly somewhat more complex. The most interesting feature of these results is once again the appearence of circular dichroism. Columns 3 and 4 of Table VII give the values for the polarization parameters where beam 1 is plane polarized, and beam 2 is circularly polarized with either left- or right-handed helicity. While the even- $j$ values of $A^{(j ; p)}$ are the same for either handedness, the odd- $j$ values change sign when the helicity is reversed. Hence, chiral discrimination is manifest; obviously the same remarks apply to the case where beam 1 is circularly polarized and beam 2 plane polarized. Equally comparing columns 5 and 6 illustrates a circular dichroism associated with two circularly polarized beams of the same handedness; once again reversing the helicity of the entire radiation field changes the sign of the odd $-j$ polarization parameters. These manifestations of chirality are only observed for the case where the two centers $A_{1}$ and $A_{2}$ have a fixed mutual orientation; it is also true that the corresponding molecular properties $T^{(1 ; q)}$ and $T^{(3 ; q)}$ disappear unless the two centers are dissymmetrically juxtaposed. The result is therefore a circular dichroism associated with the well-known coupled-oscillator model of a chiral system (Barron 1982). Not surprisingly, the chirality disappears when $A_{1}$ and $A_{2}$ are free to rotate independently, since as noted above the odd $-j$ terms then vanish.

Since the circular dichroism is generally associated with coupled groups in the near-zone range of distances, the explicit results for the Kuhn dissymmetry factors can be obtained from the leading $j_{1} / j_{0}$ terms in the rate expressions, and are as follows:

$$
\begin{aligned}
g_{1} & \equiv \frac{\Gamma(p, L)-\Gamma(p, R)}{\frac{1}{2}[\Gamma(p, L)+\Gamma(p, R)]} \\
& =\frac{\left(\omega_{1}-\omega_{2}\right) R\left(T^{(1 ; 1)}-T^{(1 ; 3)}-T^{(1 ; 4)}-2 T^{(1 ; 5)}-T^{(1 ; 6)}\right)}{c\left(T^{(0 ; 1)}+6 T^{(0 ; 2)}+T^{(0 ; 3)}\right)} \\
g_{2} & \equiv \frac{\Gamma(L, L)-\Gamma(R, R)}{\frac{1}{2}[\Gamma(L, L)+\Gamma(R, R)]} \\
& =\frac{\left(\omega_{1}-\omega_{2}\right) R\left(T^{(1 ; 2)}+T^{(1 ; 3)}+T^{(1 ; 4)}+T^{(1 ; 5)}\right)}{c\left(2 T^{(0 ; 1)}-3 T^{(0 ; 2)}-3 T^{(0 ; 3)}\right)}
\end{aligned}
$$


Here $\Gamma(p, L)$ refers to the rate of absorption w:th beam 1 plane polarized and beam 2 left-handedly polarized, and so forth. The molecular parameters, $T^{(j ; q)}$, appearing in Eqs. (9.17) and (9.19) are different for the two cases (cooperative and distributive), and their values for the particular process under study can be read off from Tables II and IV with the limiting short-range form of the interaction potential given by Eq. (8.1). Again we note the linear dependence on the group separation in Eqs. (9.17) and (9.19).

\section{Laser-Induced Circular Dichroism}

Finally, consideration is given to the results appearing in column 7 of Table VII, which apply to the situation in which the two laser beams have circular polarizations of opposite handedness. The results here differ from those in either columns 5 or 6 in each value of $j$. This represents the fact that changing the helicity of one beam produces a dichroism associated with a discrimination of the handedness of the $A_{1}-A_{2}$ pair dressed by the chirality of the other circularly polarized beam. This again is a known feature of two-photon absorption (Thirunamachandran 1979), and one which persists even when the pair is not held in a fixed mutual orientation. In this case the dissymmetry factor has both numerator and denominator given by the leading $j_{0}$ terms, and the explicit result is as follows:

$$
\begin{aligned}
g_{1} & \equiv \frac{\Gamma(L, L)-\Gamma(L, R)}{\frac{1}{2}[\Gamma(L, L)+\Gamma(L, R)]} \\
& =\frac{5\left(T^{(0 ; 3)}-T^{(0 ; 1)}\right)}{\left(T^{(0 ; 1)}+6 T^{(0 ; 2)}+T^{(0 ; 3)}\right)}
\end{aligned}
$$

\section{RESONANCE ENHANCEMENT}

Since the rate of bimolecular mean-frequency absorption will usually be somewhat less than the rate of two-photon abso-ption at any single center, the various possibilities of utilizing resonance er hancement to increase the rate of the synergistic process merit serious consideration. Resonance enhancement results from the fact if there is a sutable spacing of molecular energy levels not directly involved in the excitation scheme, a substantial increase in the absorption rate can nonetheless ensue, due to the effects of dispersion in the molecular susceptibility tensors In order to determine the conditions under which resonance enhancemen occurs it is necessary to return to the definitions of the molecular response tensors $\mathbf{S}^{f 0}$ and $\chi^{f 0}$ given by Eqs. (5.15) and (5.16) respectively. Enhancement occurs where one or more of the denominators in these equations afproaches zero, although 

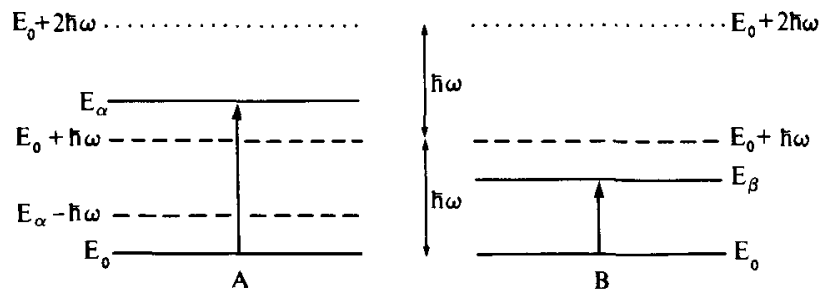

Figure 10. Resonance energy levels for single-beam synergistic absorption. The levels indicated by dashed lines represent resonances that can be exploited in absorption based on either the cooperative or the distributive mechanism; the dotted lines represent a resonance condition that applies to only the distributive case.

phenomenological damping factors, omitted here for clarity, in fact prevent infinite response at resonance.

For the single-beam cases, where the laser photon frequency is at the mean of two molecular transition frequencies, resonance enhancement occurs if either species involved in the process has an energy level matching one of those indicated in Fig. 10 by the broken lines. In the double-beam cases, the requirement for the mean of the two laser frequencies to match the molecular transition frequency provides additional freedom for one of the lasers to be tuned to one of the resonance levels shown in Fig. 11. Below we examine each case in more detail.

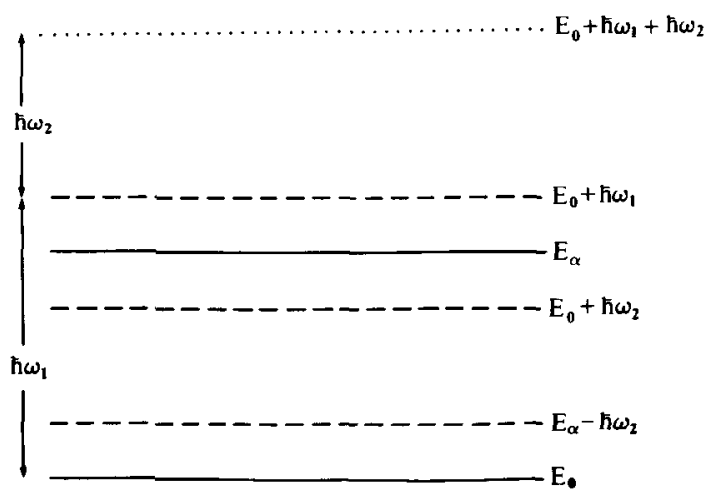

Figure 11. Resonance energy levels for two-beam synergistic absorption by pairs of chemically identical molecules. The levels indicated by dashed lines represent resonances that can be exploited in absorption based on either the cooperative or the distributive mechanism; the dotted line represents a unique resonance condition that applies to only the distributive case. 


\section{A. Single-Frequency Exciration}

Considering first the cooperative mechanism, it is clear that there are two possible resonance mechanisms to consider for the tensor $S^{\alpha 0}$ as given by Eq. (5.15), corresponding to the cases where either the first or second term dominates the expression. Thus the first term doninates if there exists a state $|r\rangle$ such that $E_{\alpha r} \approx \hbar \omega$, i.e., where $E_{r} \approx\left(E_{\alpha}-\hbar \omega\right)$. Similarly, the second term dominates if there is a state such that $E_{r 0} \approx \hbar \omega$, i.e., where $E_{r} \approx\left(E_{0}+\hbar \omega\right)$. In principle, similar remarks apply to $S^{\beta 0}$, except that if $E_{x 0}>E_{\beta 0}$ as in Fig. 10, then the condition $E_{\beta s} \approx \hbar \omega$ cannot be satisfied for any $|s\rangle$ if the

TABLE VIII

Resonance Conditions for Synergistic Photoabsorption ${ }^{a}$

\begin{tabular}{llll}
\hline & $\begin{array}{l}\text { Resonant } \\
\text { intermediate } \\
\text { energy levels }\end{array}$ & $\begin{array}{l}\text { Additional } \\
\text { conditions }\end{array}$ & $\begin{array}{l}\text { Other processes favored } \\
\text { by position of intermediate } \\
\text { energy level }\end{array}$ \\
\hline
\end{tabular}

Single-beam cooperative mechanism

$\begin{array}{llll}S^{\alpha 0}(\omega) & E_{r} \approx E_{\alpha}-\hbar \omega & \left.\text { (if } E_{\alpha}>E_{\beta}\right) & \\ S^{\beta 0}(\omega) & E_{r} \approx E_{0}+h \omega & & \text { Single-photon absorption } \\ & E_{r} \approx E_{\beta}-\hbar \omega & \left.\text { (if } E_{\beta}>E_{\alpha}\right) & \\ & E_{r} \approx E_{0}+\hbar \omega & & \text { Single-photon absorption }\end{array}$

Single-beam distributive mechanism

$\begin{array}{ll}\left.\chi^{\alpha 0}(A, \omega, \omega) \quad \quad \text { (if } E_{\alpha}>E_{\beta}\right) \\ & E_{\mathrm{s}} \approx E_{\alpha}-\hbar \omega E_{\alpha}-2 \hbar \omega \\ & E_{r} \approx E_{0}+h \omega \\ & E_{\mathrm{s}} \approx E_{0}+2 \hbar \omega\end{array}$

Double-beam cooperative mecharism
$S^{\alpha 0}\left(\omega_{1}\right)$
$E_{\mathrm{r}} \approx E_{\alpha}-\hbar \omega_{1}$
$E_{r} \approx E_{0}+h \omega_{1}$
(if $\omega_{1}<\omega_{2}$ )
$S^{\alpha 0}\left(\omega_{2}\right)$
$E_{\mathrm{r}} \approx E_{\mathrm{z}}-\hbar \omega_{2}$
$E_{\mathrm{r}} \approx E_{0}+h \omega_{2}$
(if $\omega_{2}<\omega_{1}$ )
Single-photon absorption
Single-photon absorption

Double-beam distributive mechanism

$$
\begin{array}{rlr}
\chi^{\alpha 0}\left(A, \omega_{1}, \omega_{2}\right) & E_{s} \approx E_{\alpha}-\hbar \omega_{1} & \text { (if } \left.\omega_{1}<\omega_{2}\right) \\
& E_{s} \approx E_{\alpha}-h \omega_{2} & \text { (if } \left.\omega_{2}<\omega_{1}\right) \\
& E_{r} \approx E_{\alpha}-\hbar \omega_{1}-\hbar \omega_{2} & \\
& E_{r} \approx E_{0}+\hbar \omega_{1} & \\
& E_{r} \approx E_{0}+\hbar \omega_{2} & \\
E_{s} \approx E_{0}+\hbar \omega_{1}+h \omega_{2} &
\end{array}
$$

aPositions of intermediate energy levels that produce rescinances in the molecular tensors, conditions imposed by energy constraints, and the nature of sompeting absorption processes 
initial state of $B$ is the ground state. Hence, for the single-beam cooperative case, there are just three possibilities, as indicated by the broken lines below the $E_{\alpha}$ level in Fig. 10. Clearly if $E_{\beta 0}>E_{\alpha 0}$ there are two resonance conditions that may apply for center B, and one for A. These possibilities are summarized in Table VIII.

The two conditions where $\mathbf{A}$ has a state $|\boldsymbol{r}\rangle$ or $\mathbf{B}$ has a state $|s\rangle$ with energy $\sim\left(E_{0}+\hbar \omega\right)$ lead to resonance enhancement provided the corresponding transition moments $\mu^{r 0}, \mu^{s 0}$ are non-zero. However, in such circumstances real transitions to these states are allowed by single-photon absorption, and it is likely that these effects will swamp observation of the cooperative process under consideration. The third resonance condition, where $E_{\alpha r} \approx \hbar \omega$, is of much more interest; this corresponds to having an energy level of center $A$ at approximately $\left(E_{\alpha}-\hbar \omega\right)$. Here, provided the resonant level lies sufficiently far above the ground state not to be appreciably thermally populated, then there is no question of single-photon absorption from the laser beam at frequency $\omega$, but there is nonetheless a genuine resonance amplification of the tensor $S^{\alpha 0}$. Observation of the cooperative absorption process is therefore facilitated if the energy levels of $A$ happen to lie in positions which favor this possibility.

For the distributive mechanism, the possibilities for resonance behavior can be ascertained by reference to the form of the molecular response tensor $X^{\alpha 0}(\omega, \omega)$ as given by Eq. (5.16). As noted earlier, the same tensor is involved in hyper-Raman scattering, where a figure of $10^{6}$ has been given as the order of enhancement under typical resonance conditions (Long and Stanton 1970). Clearly such a large increase will be a significant consideration in experimental studies. There are now, in principle, four different denominator factors to consider, two of which coincide with those of the cooperative mechanism and lead to the same resonance conditions. Although one additional type of resonance is predicted for states $|r\rangle$ of energy $\left(E_{\alpha}-2 \hbar \omega\right)$ or $\left(E_{\beta}-2 \hbar \omega\right)$, it is clear from the energy level diagram that neither condition can arise if the concerted absorption process takes place from the ground state.

The only other new type of resonance behavior specifically associated with the distributive case is one that applies to intermediate states $|s\rangle$ with energy $E_{s} \approx\left(E_{0}+2 \hbar \omega\right)$. However, this leads to competition from simple two-photon absorption $|s\rangle \leftarrow|0\rangle$, so that again the synergistic effect would most likely be swamped, particularly if $2 \hbar \omega$ exceeds the ionization energy of A or B. Thus it transpires that for single-beam synergistic absorption mediated by either mechanism, the most useful resonance condition corresponds to the case where there is a state of energy $\sim\left(E_{\alpha}-\hbar \omega\right)$, where there is no possibility of competition from either single-photon or two-photon absorption, and significant rate increases can be expected. 


\section{B. Two-Frequency Excitation}

For two-frequency synergistic absorption, the various resonance possibilities are also summarized in Table VIII. For cooperative mean-frequency absorption, there are again three distinct possibilities for resisnance enhancement of the process, as shown by the lowest three broken lires in Fig. 11. If the centers involved in the process possess energy levels close $10\left(E_{0}+\hbar \omega_{1}\right)$ or $\left(E_{0}+\hbar \omega_{2}\right)$, then although the molecular tensors $S^{\alpha 0}\left(\omega_{1}\right)$ or $S^{\alpha 0}\left(\omega_{2}\right)$ are resonantly enhanced, direct competition from single-photon absorption will mask the synergistic process. However, in the third resonance condition, where a molecular energy level exists close to $\left(E_{\alpha}-\hbar \omega_{2}\right)$ (assuming that $\omega_{2}$ is the lower of the laser frequencies), then the first term of $S^{\alpha 0}\left(\omega_{2}\right)$, as given by the $\omega_{2}$ analogue of Eq. (5.15), becomes resonantly enhanced and thus leads to an increased cooperative absorption rate. In this case, again provided the resonant level is not significantly populated, there is no possibility of competition from one-photon absorption.

In the two-frequency distributive case, the molecular tensor $\chi^{\alpha 0}\left(\omega_{1}, \omega_{2}\right)$ has resonance conditions similar to those for $\chi^{\alpha 0}(\omega, \omega)$. As in the single-beam case, two of the proposed resonance conditions would be likely to allow the process to be masked by single-photon absorption; a third leads to the possibility of conventional two-photon absorption, and a fourth cannot be satisfied if the centers involved are initially in their ground states. The remaining condition $\left(E_{s} \approx\left(E_{\alpha}-\hbar \omega_{1}\right)\right.$ if $\omega_{1}\left\langle\omega_{2}\right.$, or $E_{s} \approx\left(E_{\alpha}-\hbar \omega_{2}\right)$ if $\omega_{2}<\omega_{1}$ ) remains the only truly useful resonance. Naturally, since the energetics of the excitation process are constrained only by a condition on the sum of the photon frequencies, there is a wide scope for choosing laser frequencies specifically with the aim of exploiting this type of resonance possibility.

\section{COMPARISON WITH CONVENTIONAL TWO-PHOTON ABSORPTION}

The rate of conventional (single-center) two-photon absorption depends on the square of the focussed laser intensity, and as long ago as 1968 Gontier and Trahin showed that in the absence of accidental resonances an intensity factor of $\left(I / I_{o}\right)$ is introduced for each additional photon involved in a multiphoton atomic excitation process. The constant $I_{o}$ is a characteristic irradiance whose value depends on the samplis, and corresponds to the situation where perturbation theory breaks down and all multiphoton processes become equally feasible. A similar trea ment of molecules leads to an intensity factor per photon of $\gamma=\left(I / I_{M}\right)$, where $I_{M}$ is an irradiance that would lead to ionization or dissociation, and wo sld therefore have a typical 
value in the region of $10^{18 \pm 4} \mathrm{Wm}^{-2}$ (Eberly et al. 1987). This figure certainly exceeds the level of irradiance applied in most laser spectroscopy experiments, where values of $\gamma$ seldom exceed $10^{-4}$. Nonetheless two-photon spectra are readily obtained even with appreciably lower intensities. With this in mind, it is instructive to compare the likely rates of conventional and nearest-neighbor synergistic two-photon absorption.

Only by experiment or detailed $a b$ initio calculations can quantitative values be obtained for the various tensor parameters involved in the rate equations. Unfortunately, there are no studies to date which have reported the necessary numerical values, although the synergistic effects are now experimentally well-documented. Estimation of the more general significance of many of the results presented in previous sections must therefore proceed from a different basis. As shown in early work on cooperative photoabsorption (Andrews and Harlow 1983), neighboring molecules can in fact be expected to display a synergistic absorption rate approaching the rate of two-photon absorption by individual molecules, a result which is more readily calculated. This can be argued as follows. A comparison of the short-range limit of the rate equation for cooperative absorption and the corresponding rate equation for normal two-photon absorption shows that the former contains an additional factor of the order of $\rho=S^{\alpha 0} / R^{3}$. Far from accidental resonances, the molecular tensor should be similar in magnitude to the polarizability, since it is constructed in the same way from products of electric dipole transition moments divided by energy mismatch factors. Molecular polarizabilities, at least for small molecules, have well-documented values, and are mostly similar in magnitude to the cube of molecular diameter. Hence when $R$ represents a nearest-neighbor distance, the factor $\rho$ approaches the value of unity, and the cooperative absorption rate is comparable with that of conventional two-photon absorption. Similar arguments apply in the case of distributive absorption.

Obviously, any possibilities of resonance, as discussed in Section X, can further enhance the synergistic photoabsorption rates. While most of the appropriate resonance conditions are held in common with single-center two-photon absorption, the case of resonance at a level one photon in energy below the final excited state of either molecule participating in a synergistic process is a unique feature. This not only opens up important new possibilities for rate enhancement, but it does so without the associated complications of competing absorption processes. Thus it appears that synergistic effects should be generally observable at the levels of laser irradiance typically employed for studies of conventional two-photon absorption, and it should not be necessary to utilize exceptionally intense laser sources where higher-order optical nonlinearities might become a problem. 


\section{SYNERGISTIC EFFECTS IN THE ABSORPTION OF WHITE LIGHT}

\section{A. Spectrophotometry with Broadband Sources}

Having established the detailed theory underlying synergistic effects in two-photon laser spectroscopy, we now consider the broader implications of these effects in connection with conventional absorption processes. Most modern spectrophotometry and color science is based on the principle that the optical response of a substance to a given wavelength of light is independent of any other wavelengths that may be: present. Thus it is normally assumed that an absorption spectrum obtained using broadband light and a multichannel spectrometer would be identical to the spectrum obtained using a tunable monochromatic light source. Indeed, this is the principle underlying Fourier transform spectroscopy.

Using results established in previous sections, it can however be shown that this assertion represents only an approximation to the truth. Absorption from a white light or other broadband source in fact allows photon pairs of differing frequencies to be concertedly absorhed by molecules in close proximity (Andrews 1988). In this section, it is shown that this may result in a change to the appearance of absorption spectra, and yield an absorption law that departs from normal Beer-Lambert behavior. One particular case in which such effects may be expected to arise is where supercontinuum laser radiation is employed for spectroscopic purposes.

The generation of a white-light supercontinuum by passing mode-locked pulses of laser light through certain media was first reported in 1970 (Alfano and Shapiro 1970). The phenomenon results from a process of self-phase modulation associated with intensity-dependent refraction, although a number of other mechanisms can contribute to the effect; a useful summary is provided in a recent review by Alfano (1986). Continuum generation has been shown to occur in a wide variety of materials, and is readily producible in water. The pulses of light so generated are often referred to as constituting an ultrafast supercontinuum laser source (USLS) (Manassah et al. 1984), or picosecond continuum for short, since pulse durations are typically on the picosecond or femtosecond (Fork et al. 1983) timescale. The term superbroadening is also used to describe the continuum formation (Reintjes 1984).

The laser supercontinuum source has found numerous applications in the physical, chemical, and biological sciences. Many studies have concerned elementary photochemical and photobiological reactions such as those involved in the primary processes of photosyr.thesis and vision (von der 
Linde 1977; Eisenthal 1977; Peters and Leontis 1982; Fleming 1986). These studies are mostly based on use of the continuum to probe the absorption characteristics of transient species, in order to obtain information on their decay kinetics. The processing of data from such experiments is generally based on the implicit assumption that the absorption of white light is subject to the normal Beer-Lambert law, in that the absorption at any particular frequency is assumed to be linearly proportional to the intensity of the probe light at that frequency.

\section{B. Rate Equations for Continuum Excitation}

To place into proper perspective the role of synergistic effects in the absorption of white light, it is worth first setting down the basic equations for the normal absorption process observed with monochromatic light. Consider an ensemble of molecules in an initial state $|i\rangle$, certain of which are promoted to an excited state $|f\rangle$ through absorption of light with circular frequency $\omega_{0}$, i.e., we have $E_{f i}=\hbar \omega_{0}$. Assuming that the transition is electric dipole-allowed, the rate of (single-photon) absorption is given by;

$$
\Gamma_{1}=\left(\hbar^{2} c \varepsilon_{0}\right)^{-1} \pi K_{1} l\left(\omega_{0}\right)
$$

where $l\left(\omega_{0}\right)$ is defined as the irradiance per unit circular frequency $(\omega)$ interval at frequency $\omega_{0}$, and $K_{1}$ is given by

$$
K_{1}=\left|\boldsymbol{\mu}^{f i} \cdot \mathbf{e}\right|^{2}
$$

$\boldsymbol{\mu}^{f i}$ being the transition dipole moment for the $|f\rangle \leftarrow|i\rangle$ transition, and $\mathrm{e}$ the unit polarization vector of the incident light.

The result of Eq. (12.1) is more often expressed in terms of the Einstein $B$-coefficient; casting the result in terms of radiant energy density per unit frequency $(\omega)$ interval introduces an additional factor of $c / 2 \pi$, and a further factor of $\frac{1}{3}$ results from rotational averaging. However, to facilitate subsequent comparison with the rate equation for cooperative absorption, the above result is given in the form it takes prior to rotational averaging. The single most important feature to note at this stage is the linear dependence of the absorption rate on the irradiance, a dependence manifest in the characteristic exponential decay of intensity with time and hence also with distance travelled through the sample (the Beer-Lambert Law).

For cooperative absorption, two photons with frequencies $\omega=\left(\omega_{0}+\Omega\right)$ and $\omega^{\prime}=\left(\omega_{0}-\Omega\right)$, the sum of whose energies equals the sum of the $|f\rangle \leftarrow|i\rangle$ transition energies for two different molecules, are absorbed in the concerted process illustrated by Fig. 2 . The rate of absorption by the pair is essentially 
that given by Eq. (6.9), recast in terms of an irradiance with a large bandwidth:

$$
\Gamma_{2 c}=\left(2 \hbar^{2} c^{2} \varepsilon_{0}^{2}\right)^{-1} \pi \int_{0}^{\infty} K_{2}\left(\omega_{0}, \Omega\right) l\left(\omega_{0}+\Omega\right) l\left(\omega_{0}-\Omega\right) d \Omega
$$

where

$$
K_{2}\left(\omega_{0}, \Omega\right)=\left|e_{i} e_{j} S_{i k}^{f i}\left(\omega_{0}+\Omega\right) S_{j l}^{f i}\left(\omega_{0}-\Omega\right)\left\{V_{k l}(\Omega, \mathbf{R})+\bar{V}_{k l}(\Omega, \mathbf{R}) \exp (i \Delta \mathbf{k} \cdot \mathbf{R})\right\}\right|^{2}
$$

The result is simplified by the physically reasona ole assumption that the two absorbed photons have the same polarization.

To the extent that the parameter $K_{2}\left(\omega_{0}, \Omega\right)$ is approximately frequencyindependent over any range of frequencies well away from resonance (in other words, whenever dispersion effects are small), the rate contribution $\Gamma_{2 c}$ has a direct dependence on the frequency-domain autocorrelation function of the incident light. Since USLS radiation is pulsed, it is useful to express the result in terms of the time-dependence of the irradiance $I(t)$ through the Fourier transform

$$
l(\omega)=(2 \pi)^{-1 / 2} \int_{-\infty}^{\infty} I(t) \exp (i \omega t) d t
$$

Simple manipulation of the integral in Eq. (12.3) then reveals its equivalence to the time-domain integral

$$
K_{2}\left(\omega_{0}\right) \int_{-\infty}^{\infty} I^{2}(t) \exp \left(2 i \omega_{0} t\right) d t
$$

In the distributive mechanism two photons with frequencies $\left(\omega_{0}+\Omega\right)$ and $\left(\omega_{0}-\Omega\right)$ undergo concerted absorption at the same center, and the energy mismatch $E_{f i}$ is conveyed to another molecule by virtual photon coupling, as in Fig. 3. In this case, using exactly similar methods, the following rate equation is obtained:

$$
\Gamma_{2 d}=\left(2 \hbar^{2} c^{2} \varepsilon_{0}^{2}\right)^{-1} \pi \int_{0}^{\infty} K_{2^{\prime}}\left(\omega_{0}, \Omega\right) l\left(\omega_{0}+\Omega\right) l\left(\omega_{0}-\Omega\right) d \Omega
$$

where

$$
K_{2},\left(\omega_{0}, \Omega\right)=\left|e_{i} e_{j} \chi_{(i j) k}^{f i}\left(\omega_{0}+\Omega, \omega_{0}-\Omega\right) \mu_{l}^{f i} V_{k l}\left(\omega_{0}, \mathbf{R}\right)\{1+\exp (i \mathbf{u} \cdot \mathbf{R})\}\right|^{2}
$$


and once again a link can be established with the autocorrelation function of the USLS light.

The Beer-Lambert exponential decay law for conventional (singlephoton) absorption results from the elementary relation

$$
-d l(\omega, z) / d z \propto l(\omega, z)
$$

where $z$ represents the distance the light has travelled through the absorbing sample. Since this is directly proportional to the propagation time within the sample, Eq. (12.9) is a result which follows directly from Eq. (12.1). When intense continuum light such as that provided by USLS radiation is absorbed, cooperative and distributive processes produce a correction term that necessitates the replacement of Eq. (12.9) by a result of the form

$$
-d l(\omega, z) / d z \propto\left[l(\omega, z)+\chi \int K(\omega, \Omega) l(\omega+\Omega, z) l(\omega-\Omega, z) d \Omega\right]
$$

where $K=K_{2}+K_{2}$. Clearly, in this case exponential decay is no longer to be expected.

One of the most significant implications of the result is that an absorption spectrum measured with intense white light may be significantly different from the spectrum that would be observed using tunable monochromatic radiation. In particular, there should be a decrease in the apparent width of many lines in any absorption spectrum measured with broadband radiation. This is because, for any sample transition of frequency $\omega_{0}$, photons of appreciably off-resonant frequency $\left(\omega_{0} \pm \Omega\right)$ can be cooperatively absorbed and result in the excitation of two separate molecules, provided selection rules permit. In fact the Lorentzian linewidth of the concerted absorption process is readily shown to be approximately $0.64 \times$ the ordinary absorption linewidth, if the probe radiation is assumed to be of nearly constant intensity in the frequency region of interest. Nonetheless, the observed linewidth would not be reduced to quite this extent, because of the additional and invariably stronger response associated with normal single-photon absorption.

\section{Implications for Spectroscopy with USLS Radiation}

It is difficult in general terms to assess the magnitude of the correction represented by the frequency integral, although under optimum conditions it may indeed be comparable with the first term in the square brackets (Andrews 1988). The most significant feature of Eq. (12.10) is undoubtedly the fact that the absorption by a sample at any given frequency is directly influenced by the intensity of light at other frequencies. Equation (12.10) thus represents an infinite set of coupled integro-differential equations, whose solution depends on the detailed spectral distribution of the USLS continuum 
light (Manassah et al. 1985a, b, 1986; Manassah 1986) and also the spectral response of the sample, as represented by the ail-embracing constant $K$.

Precise values for the focused irradiances produced by USLS pulses are not currently available in the literature, but the intensity levels are certainly high enough for two-photon absorption to be experimentally observable, and by extension it appears that the synergistic process may produce important contributions to conventional single-photon absorption spectra. Other factors that contribute to the significance of cooperative or distributive two-photon absorption are the involvement of non-neighboring molecules, and any enhancement of the molecular response tensors through incidental one-photon resonances, as discussed in Section X. In many situations, it is therefore likely that mean-frequency absorption will play a significant role in modifying the apparent form of absorption spectra.

As noted above, the use of USLS light for probing absorption may result in a modified linewidth in the spectrum. Other more significant changes may also be expected, however. This can be illustrated as follows. Consider the case of an electronic transition which displays vibronic structure associated with a certain molecular vibration. For simplicity, let us confine attention to the $(0-0)$ band, assuming that the vibrational frequencies in the ground and excited electronic states are similar. Although cooperative and distributive processes allow the absorption of any pair of photons whose energy sum equals that of the $(0-0)$ excitation energies for two different molecules, the rate of each process is resonantly enhanced if either photon energy matches that of another transition.

In particular, a pair of photons whose energies match the $(0-1)$ and $(1-0)$ transitions can be cooperatively absorbed and so actually result in $(0-0)$ transitions in two separate molecules. Thus, because of the resonance enhancement associated with a (0-1)-frequency photon, one should expect increased absorption at both the (0-1) and (1-0) frequencies, even if the $v=1$ level in the electronic ground state is not appreciably populated. Features of this kind have been noted in recent USLS-probe experiments on spectral hole burning in dye solutions (Alfano et al. 1974). Although it is unlikely that cooperative absorption has any direct bearing on these studies in view of the large mean separation of the dye molecules in solution, it might be expected to become much more significant in studies of molecular crystals, for example.

In assessing the wider significance of mean-frequency absorption for flash photolytic experiments based on USLS radiation, perhaps the most important factor to consider is the enormously wide range of possibilities for synergistic absorption leading to the simultaneous excitation of more than one excited state. For example, with a single-component sample, it should be possible to observe the process

$$
2 \mathrm{~A}+\hbar \omega+h \omega^{\prime} \rightarrow \mathrm{A}^{*}+\mathrm{A}^{\ddagger}
$$


the double dagger denoting some other excited state than that denoted by the asterisk. If the sample is heterogeneous or contains more than one chemical species, there exists the even more general possibility of simultaneously exciting two chemically different species:

$$
\mathrm{A}+\mathrm{B}+\hbar \omega+\hbar \omega^{\prime} \rightarrow \mathrm{A}^{*}+\mathrm{B}^{*}
$$

The reaction represented by Eq. (12.12) is potentially very significant for flash photolytic studies of processes in complex biological systems, where A and B may even be chemically different chromophores within a single large biomolecule. The theory underlying these processes can be developed from the basic results in Section V, and the associated rates of absorption should be comparable to those of the closely related synergistic absorption processes discussed earlier in this review. However, in polyatomic molecules with complex vibronic structures, the number of pairs of transitions that can be excited through absorption of two photons with the correct energy sum may be enormously large, so that cooperative absorption may exert a very significant effect on the appearance of the absorption spectrum. The numerous possibilities for resonance enhancement of the molecular response tensors at certain frequencies should also be borne in mind.

In conclusion, it is worth reiterating that the anomalous absorption effects described here may be manifest in any experiments that employ sufficiently high-intensity broadband radiation. To this extent, anomalies may be observable in experiments not specifically involving USLS light. In particular, the continued advances in techniques of laser pulse compression have now resulted in the production of femtosecond pulses only a few optical cycles in duration (Knox et al. 1985; Brito Cruz et al. 1987; Fork et al. 1987) which necessarily have a very broad frequency spread, as the time/energy uncertainty principle shows. Thus, mean-frequency absorption may have a wider role to play in the absorption of femtosecond pulses. If this is correct, it raises further questions over the suitablity of absorption-based techniques for their characterization.

\section{CHEMICAL ASPECTS OF SYNERGISTIC PHOTOABSORPTION}

\section{A. General Considerations}

Although the most thoroughly documented studies of synergistic two-photon absorption relate to matrix isolation studies, the process should certainly be manifest in other phases of matter. For samples in either a gas or liquid state, it might be argued that such effects are essentially collisional phenomena. It is certainly true that the probability of any such synergistic 
process is very significantly enhanced for pairs of molecules separated by small distances, a dependence which must be associated with a high degree of pressure sensitivity. However, although these processes are predominantly effective over short distances, they are nonetheless induced by proximity rather than collision. This much is made clear by the fact that irrespective of any energy shifts or selection rule weakening which collisions might induce, the absorption processes that occur at each center are separately forbidden on energy grounds: moreover, there are significant contributions from molecules in the far-zone region. By the same token, orbital overlap need not be implicated in synergistic processes involving chromophore pairs.

The synergistic processes described in this review can potentially be manifest in a very wide range of chemical and physical effects, and we now turn to a consideration of some of the broader implications. Although there has been tacit assumption through most of our discussion that the excitation of the two centers is electronic or vibronic in nature, purely vibrational excitation is also possible if suitable wavelengths are employed, and suitable Born-Oppenheimer development of the molecular tensors enables the theory to be directly applied to such cases. In the case where both centers gain one quantum of vibrational energy, then the irradiation frequency for the synergistic single-beam process is the mean of these two vibrational frequencies. Vibrational modes that can participate in this process by the cooperative mechanism must be Raman-allowed by virtue of the two-photon selection rules; in the distributive case, the vibrations must be infrared-allowed. In both cases, the limiting near-zone behavior applies up to distances comparable to infrared wavelengths. In the case of two-beam excitation, similar remarks apply.

In considering the single-beam cases, moreover, we have concentrated on the case where A and B differ, even if only by isotopic constitution. However, the theory can also apply to the case where $A$ and $B$ are identical, but the excited states $|\alpha\rangle$ and $|\beta\rangle$ differ. For a single-component phase, the effect should be observable as the absorption of two photons with energy halfway between that of the two excited states; this appiies equally to free molecules or loosely associated van der Waals dimers. For a two-component phase containing a mixture of $A$ and $B$ molecules, it should thus be possible to observe synergistic effects associated with not only $\mathbf{A}-\mathbf{B}$, but also $\mathbf{A}-\mathbf{A}$ and $B-B$ interactions, and hence several new bands should appear in the two-photon absorption spectrum.

\section{B. Methods of Observation}

We now consider some of the methods by which the synergistic effects described above may be observed. As with conventional two-photon absorption, direct monitoring of a reduction in laser intensity as a result of 
the photoabsorption process is unlikely to be the most practicable method for studying the effect. However, fluorescent decay from any of the excited states is much more easily measured since it should be against a zero background; note that fluorescence from only one excited species is normally sufficient to demonstrate the occurrence of a synergistic process. For the sake of argument, let us suppose that the excitation of the state $|\alpha\rangle$ is monitored. Radiationless decay within the vibrational manifold of the initially populated electronic state will generally mean that the fluorescence will be Stokes-shited in frequency away from $E_{\alpha 0} / h$. Nevertheless, since the same excited state $|\alpha\rangle$ is also accessible either through single-photon absorption at frequency $E_{\alpha 0} / h$ (in the distributive case), or through single-beam two-photon absorption at frequency $E_{\alpha 0} / 2 \hbar$ (in the cooperative case), its decay characteristics can be ascertained in a separate single-beam experiment and then used to monitor the synergistic effect.

Other methods can be devised according to the photochemistry of the sample, and as an example we consider the case of a mixture of $\mathrm{H}_{2} \mathrm{CO}$ and $\mathrm{D}_{2} \mathrm{CO}$. These molecules have absorption lines associated with the $2{ }_{0}^{1} 4_{0}^{3}$ transition at 30,340.15 and 30,147.62 $\mathrm{cm}^{-1}$, respectively (Moule and Walsh 1975). Irradiation with a single beam of laser light at the mean of these two frequencies should thus lead to a synergistic two-photon process, involving both the cooperative and distributive channels, in which both species are simultaneously excited. The narrow bandwidth of any standard laser source should ensure that neither species is independently excited by a conventional single-photon absorption process. Evidence for cooperative two-photon absorption is then provided by detection of the decomposition product $\mathrm{CO}$ resulting from the reactions:

$$
\begin{aligned}
& \mathrm{H}_{2} \mathrm{CO}^{*}-\left[\begin{array}{l}
\mathrm{H}_{2}+\mathrm{CO} \\
\mathrm{H}+\mathrm{H}+\mathrm{CO}
\end{array}\right. \\
& \mathrm{D}_{2} \mathrm{CO}^{*}-\left[\begin{array}{l}
\mathrm{D}_{2}+\mathrm{CO} \\
\mathrm{D}+\mathrm{D}+\mathrm{CO}
\end{array}\right.
\end{aligned}
$$

The experimental verification of this reaction scheme remains one of the main challenges presented by the theory of synergistic photoabsorption.

\section{Charge Transfer Reactions}

Most of the experimental work on synergistic photoabsorption has concerned the studies of charge transfer between molecular halogens and rare gas atoms. This type of two-photon induced process can in general be expressed as

$$
X_{2}+\operatorname{Rg}+2 \hbar \omega \rightarrow \operatorname{Rg}^{+} X_{2}^{-}
$$


The subsequent reactions

$$
\begin{aligned}
& \operatorname{Rg}^{+} X_{2}^{-} \rightarrow \operatorname{Rg}^{+} X^{-}+X \\
& \operatorname{Rg}^{+} X^{-}+\operatorname{Rg} \rightarrow \operatorname{Rg}_{2}^{+} X^{-}
\end{aligned}
$$

generally lead to exciplexic emission which characterizes completion of the reaction, as illustrated in Fig. 12. Reactions of this type have been studied in gas-phase experiments (Yu et al. 1983; $\mathrm{Ku}$ et al. 1983), and in $\mathrm{Rg}: \mathrm{X}_{2}$ van der Waals complexes generated in free jet exparısions (Boivineau et al. 1986; Jouvet et al. 1987). However, the most extensive studies have been performed by Apkarian and co-workers in condensed phases, involving both liquid solutions and doped rare gas matrices (vide infra). The broad absorption by halogens in the UV-visible range offers plenty of scope for the exploitation of single-photon resonances to enhance the prospects of observing synergistic effects. The advantage is offset by the fact that these resonances are mostly associated with dissociative transitions to turning points of strongly repulsive potentials, resulting in uncorrelated absorption processes that complicate the experiments. Careful analyses have been required to prove unequivocally the

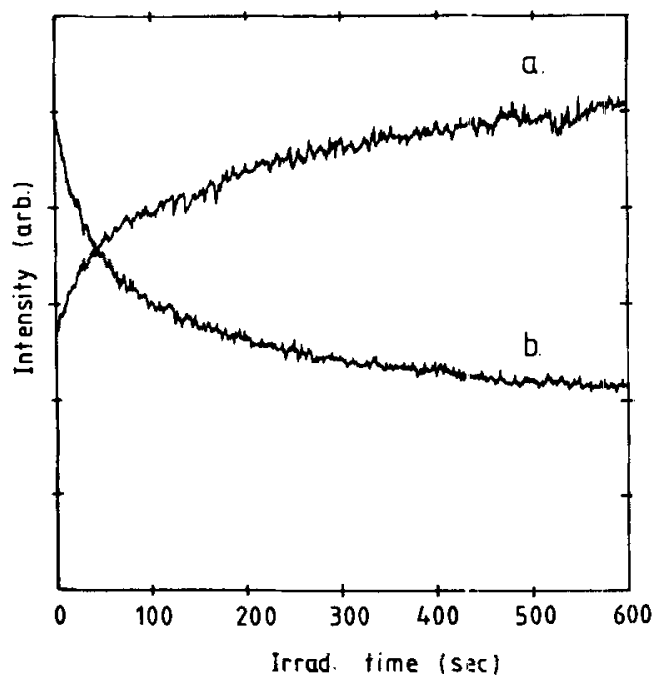

Figure 12. Permanent dissociation of $\mathrm{Cl}_{2}$ in solid xenon irradiated at $308 \mathrm{~nm}$, as a function of irradiation time (1:500 Cl 2 :Xe solid at $13 \mathrm{~K}$ ). (a) Growth o the $573 \mathrm{~nm} \mathrm{Xe}{ }_{2}{ }^{+} \mathrm{Cl}^{-}\left(4^{2} \Gamma \rightarrow 1,2^{2} \Gamma\right)$ exciplexic emission intensity. (b) Decrease of the $\mathrm{Cl}_{2}\left(\mathrm{~A}^{\prime} \rightarrow \mathrm{X}\right)$ recombinant emission intensity at $800 \mathrm{~nm}$ in the same sample. Redrawn from Fajardo, W thnall et al. (1988) by permission of Harwood Academic Publishers GmbH. 
existence of a synergistic mechanism for the charge transfer. Various nonsynergistic mechanisms can dominate under different conditions; the task has been to demonstrate the occurrence of charge transfer even under conditions where these alternative mechanisms cannot apply.

The first possibility to eliminate is the absorption of a second photon by the halogen in its photoexcited state, followed by charge transfer:

$$
\mathrm{X}_{2}^{*}+\mathrm{Rg} \rightarrow \mathrm{Rg}^{+} \mathrm{X}_{2}^{-}
$$

Working with $\mathrm{F}_{2}, \mathrm{Cl}_{2}$, and $\mathrm{Br}_{2}$ in wavelength regions where such resonances are absent, it has nonetheless been shown that the charge transfer reactions persist and cannot be accounted for by two-photon absorption in the free halogens (Fajardo and Apkarian 1988a, b). A second mechanism which could contribute is photodissociation of the molecular halogen followed by photo-induced charge transfer between the atomic species:

$$
X+\operatorname{Rg}+\hbar \omega \rightarrow \operatorname{Rg}^{+} X^{-}
$$

This latter mechanism can be ruled out in a number of ways through judicious choice of experimental conditions, as most notably demonstrated by studies of the photo-induced harpoon reactions of ICl in liquid and solid xenon (Okada et al. 1989). Here it has been shown that XeI is produced even at wavelengths well beyond the threshold for atomic charge transfer between

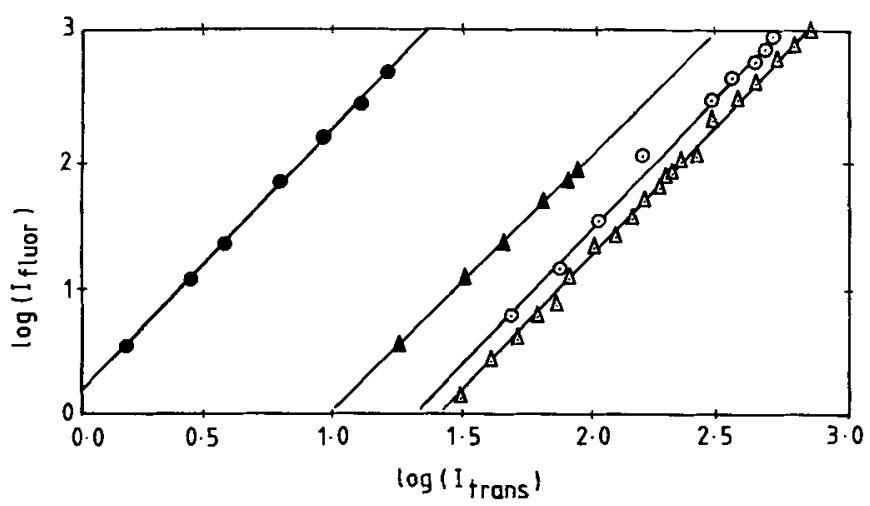

Figure 13. Logarithmic plots of exciplexic fluorescence intensities versus transmitted laser intensity in a $1.3 \mathrm{mM}$ solution of $\mathrm{Cl}_{2}$ in Xe, at various wavelengths: $340 \mathrm{~nm}$ (solid circles), $360 \mathrm{~nm}$ (solid triangles), $308 \mathrm{~nm}$ (open circles), $368 \mathrm{~nm}$ (open triangles). The corresponding slopes of the best fit lines are 2.07, 1.98, 2.11, and 2.08, respectively. Redrawn from Fajardo, Withnall et al. (1988) by permission of Harwood Academic Publishers GmbH. 
the rare gas and halogen atoms. It transpires, however, that resonances associated with the final state in $\mathrm{ICl}$ arise in accordance with the operation of a synergistic mechanism.

The explicit characterization of synergistic two-photon absorption in liquid solutions has recently been described by Fr.jardo, Withnall et al. (1988). Here a clear distinction from the effects of any sequential absorption has been made on the basis of kinetic considerations. In particular, the intensity of fluorescence by the exciplex $\mathrm{Xe}^{+} \mathrm{Cl}^{-}$ha: been shown to depend quadratically on laser fluence over a range of wavelengths, exactly as one expects with a coherent two-photon process. Any sequential mechanism would only be expected to produce this kind of dependence if the cross-sections for photodissociation and atomic charge transfer were similar in magnitude. In fact, both cross-sections vary by at least an order of magnitude over the wavelength range studied, yet the dependence on fluence satisfies a power law with exponent $2.05 \pm 0.7$ throughout, as shown in Fig. 13. Synergistic two-photon absorption is thus the only mechanism that can satisfactorily account for all the experimental observations.

\section{Acknowledgement}

We would like to express our thanks to V. A Apkarian for his detailed communication of recent experimental results on synergistic effects in photo-induced charge transfer.

\section{References}

Alfano, R. R., 1986, in Proc. Int. Conf. Lasers' 85, C. P. Wang Ed. (STS Press, McLean, Virginia) pp. 110-122.

Alfano, R. R. and S. L. Shapiro, 1970, Phys. Rev. Lett. 24, 585-587.

Alfano, R. R., J. I. Gersten, G. A. Zawadzkas, and N. Tzoar, 1974, Phys. Rev. A10, 698-708.

Andrews, D. L., 1976, Chem. Phys. 16, 419-424.

Andrews, D. L., 1984, Mol. Phys. 52, 969-972.

Andrews, D. L., 1985, Am. J. Phys. 53, 1001-1002.

Andrews, D. L., 1988, Phys. Rev. A38, 5129-5139.

Andrews, D. L., 1989, Phys. Rev. A40, 3431-3433.

Andrews, D. L. and W. A. Ghoul, 1982, Phys. Rev. A25, 26417-2657.

Andrews, D. L. and M. J. Harlow, 1983, J. Chem. Phys. 78, 1088-1094.

Andrews, D. L. and M. J. Harlow, 1984a, J. Chem. Phys. 80, 4753-4760.

Andrews, D. L. and M. J. Harlow, 1984b, Phys. Rev A29, 2796-2806.

Andrews, D. L. and K. P. Hopkins, 1987, J. Chem. Phys. 86, 2453-2459.

Andrews, D. L. and K. P. Hopkins, 1988a, J. Mol. Struc. 175, 141-146.

Andrews, D. L. and K. P. Hopkins, 1988b, J. Chem. Phys. 89, 4461-4468.

Andrews, D. L. and B. S. Sherborne, 1987, J. Chem. Phys. 86, 4011-4017.

Andrews, D. L. and T. Thirunamachandran, 1977, J. Chem. Phys. 67, 5026-5033. 
Andrews, D. L. and T. Thirunamachandran, 1978a, Proc. Roy. Soc. Lond. A358, 297-310.

Andrews, D. L. and T. Thirunamachandran, 1978b, Proc. Roy. Soc. Lond. A358, 311-319.

Andrews, D. L. and P. J. Wilkes, 1985, J. Chem. Phys. 83, 2009-2014.

Arnold, S., W. B. Whitten, and A. C. Damask, 1970, J. Chem. Phys. 53, 2878-2884.

$\mathrm{Au}$, C. K., 1988, Phys. Rev. A38, 7-12.

Avakian, R. and R. E. Merrifield, 1968, Mol. Cryst. 5, 37-77.

Barron. L. D., 1975, J. Chem. Soc. Faraday Trans. 2, 71, 293-300.

Barron, L. D., 1982, Molecular Light Scattering and Optical Activity (Cambridge University Press, Cambridge) pp. 249-263.

Barron, L. D. and A. D. Buckingham, 1974, J. Am. Chem. Soc. 96, 4769-4773.

Barron, L. D. and C. J. Johnston, 1987, Mol. Phys. 62, 987-1001.

Boivineau, M., J. leCalvé, M. C. Castex, and C. Jouvet, 1986a, Chem. Phys. Lett. 128, 528-531.

Boivineau, M., J. leClavé, M. C. Castex, and C. Jouvet, 1986b, J. Chem. Phys. 84, 4712-4713.

Boys, S. F., 1934, Proc. Roy. Soc. Lond. A144, 655-674.

Brechignac, C., Ph. Cahuzac, and P. E. Toschek, 1980, Phys. Rev. A21, 1969-1974.

Brito Cruz, C. H., R. L. Fork, and C. V. Shank, 1987, Conf. Lasers and Electro-Optics (Baltimore, Maryland).

Casimir, H. B. G. and D. Polder, 1948, Phys. Rev. 73, 360-372.

Compagno, G., R. Passante, and F. Persico, 1983, Phys. Lett. 98A, 253-255.

Compagno, G., F. Persico, and R. Passante, 1985, Phys. Lett. 112A, 215-219.

Craig, D. P. and T. Thirunamachandran, 1982, Adv. Quantum Chem. 16, 97-160.

Craig, D. P. and T. Thirunamachandran, 1984, Molecular Quantum Electrodynamics (Academic, New York).

Craig, D. P. and S. H. Walmsley, 1964, Excitons in Molecular Crystals(Benjamin, New York).

Danielson, R. E., 1974, Astrophys. J. 192, L107-L1 10.

Débarre, A. and Ph. Cahuzac, 1986, J. Phys. B19, 3965-3973.

Dexter, D. L., 1962, Phys. Rev. 126, 1962-1967.

Dodge, W. R., 1985, Nucl. Instrum. Meth. Phys. Res. B10/11, 423-431.

Eberly, J. H., P. Maine, D. Strickland, and G. Mourou, 1987, Laser Focus 23 (10), 84-90.

Eisenthal, K. B., 1977, in Ultrashort Light Pulses-Picosecond Techniques and Applications, S. L. Shapiro, Ed. (Springer-Verlag, Berlin) pp. 275-315.

Fajardo, M. E. and V. A. Apkarian, 1986, J. Chem. Phys. 85, 5660-5681.

Fajardo, M. E. and V. A. Apkarian, 1987, Chem. Phys. Lett. 134, 55-59.

Fajardo, M. E. and V. A. Apkarian, 1988a, J. Chem. Phys. 89, 4102-4123.

Fajardo, M. E. and V. A. Apkarian, 1988b, J. Chem. Phys. 89, 4124-4136.

Fajardo, M. E., R. Withnall, J. Feld, F. Okada, W. Lawrence, L. Wiedeman, and V. A. Apkarian, 1988, Laser Chem. 9, 1-26.

Feynman, R. P., 1949, Phys. Rev. 76, 769-789.

Feynman, R. P., 1961, Quantum Electrod ynamics (Benjamin/Cummings, Reading, Massachusetts).

Finkel, R. W., 1987, Phys. Rev. A35, 1486-1489.

Fleming, G. R., 1986, Chemical Applications of Ultrafast Spectroscopy (Oxford University Press, Oxford) p. 80.

Fork, R. L., C. V. Shank, C. Hirlimann, R. Yen, and W. J. Tomlinson, 1983, opt. Lett. 8, 1-3. 
Fork, R. L., C. H. Brito Cruz, P. C. Becker, and C. V. Shank, 1987, Opt. Lett. 12, 483-485.

Förster, Th., 1949, Z. Naturforsch. 4A, 321-327.

Gallagher, A. and T. Holstein, 1977, Phys. Rev. A16, 2413-.2431.

Gedanken, A. and M. Tamir, 1988, Rev. Sci. Instrum. 58, 950-952.

Geltman, S., 1976, J. Phys. B9, L569-L574.

Geltman, S., 1987, Phys. Rev. A35, 3775-3783.

Gontier, Y. and M. Trahin, 1968, Phys. Rev. 172, 83-87.

Green, W. R., J. Lukasik, J. R. Willison, M. D. Wright, J. F. Young, and S. E. Harris, 1979, Phys. Rev. Lett. 42, 970-973.

Groff, R. P., P. Avakian, and R. E. Merrifield, 1970, Phys. Fev. B1, 815-817.

Grossel, P. H., J. M. Vigoureux, and D. Van Labeke, 1983, Phys. Rev. A28, 524-531.

Haken, H., 1977, Synergetics (Springer-Verlag, Berlin).

Haken, H., 1985, Light Vol. 2: Laser Light Dynamics (North-Holland, Amsterdam) p. 9.

Jouvet, C., M. Boivineau, M. C. Duval, and B. Soep, 1987, I. Phys. Chem. 91, 5416-5422.

Ketelaar, J. A. A., 1959, Spectrochim. Acta 14, 237-248.

Kirkwood, J.G., 1937, J. Chem. Phys. 5, 479-491.

Knox, W. H., R. L. Fork, M. C. Downer, R. H. Stoler, C. V. Shank, and J. A. Valdmanis, 1985, Appl. Phys. Lett. 46, 1120-1121.

Ku, J. K., G. Inoue, and D. W. Setser, 1983, J. Phys. Chem. 87, 2989-2993.

Kuhn, W., 1930, Trans. Faraday Soc. 26, 293-308.

Last, I., Y. S. Kim, and T. F. George, 1987, Chem. Phys. Leit. 138, 225--230.

Locke, R. J. and E. C. Lim, 1987, Chem. Phys. Lett. 134, $10^{5}-109$.

Long, D. A. and L. Stanton, 1970, Proc. Roy. Soc. Lond. A318, 441-457.

Loudon, R., 1983, The Quantum Theory of Light, 2nd. edition (Oxford University Press, Oxford), pp. 105-111.

Louisell, W. H., 1973, Quantum Statistical Properties of Radiation (Wiley, New York), pp. 176-180.

Manassah, J. T., 1986, Phys. Lett. A117, 5-9.

Manassah, J. T., P. P. Ho, A. Katz, and R. R. Alfano, 1984, Photonics Spectra 18, (11), 53-59.

Manassah, J. T., R. R. Alfano, and Mustafa, M. A. 1985a, Phys. Lett. 107A, 305-309.

Manassah, J. T., M. A. Mustafa, R. R. Alfano, and P. P. Ho, 1985b, Phys. Lett. 113A, 242-247.

Manassah, J. T., M. A. Mustafa, R. R. Alfano, and P. P. Ho, 1986, IEEE J. Quantum Electron. QE-22, 197-204.

Meath, W. J. and E. A. Power, 1987, J. Phys. B20, 1945-1954.

Mohler, C. E. and M. J. Wirth, 1988, J. Chem. Phys. 88, 7369-7375.

Monson, P. R. and W. M. McClain, 1970, J. Chem. Phys. 53, 29-37.

Moule, D. C. and A. D. Walsh, 1975, Chem. Rev. 75, 67-84.

Nakazawa, E. and S. Shionoya, 1970, Phys. Rev. Lett. 25, 1710-1712.

Nosworthy, J. and J. P. Keene, 1964, Proc. Chem. Soc. 114.

Okada, F., L. Wiedeman, and V. A. Apkarian, 1989, J. Phys. Chem. 93, 1267-1272.

Parker, C. A. and C. G. Hatchard, 1962, Proc. Roy. Soc. Lond. A269, 574-584.

Peters. K. S. and N. Leontis, 1982, in Biological Events Probed by Ultrafast Laser Spectroscopy, R. R. Alfano, Ed. (Academic, New York) pp. 259-269. 
Power, E. A., 1975, J. Chem. Phys. 63, 1348-1350.

Power, E. A. and T. Thirunamachandran, 1983, Phys. Rev. A28, 2671-2675.

Power, E. A. and S. Zienau, 1959, Philos. Trans. Roy. Soc. London Ser. A251, 427-454.

Reintjes, J. F., 1984, Nonlinear Optical Parametric Processes in Liquids and Gases (Academic, Orlando, Florida) pp. 355-357.

Rios Leite, J. R. and C. B. De Araujo, 1980, Chem. Phys. Lett. 73, 71-74.

Sasaki, A. and S. Hayakawa, S. 1978, Jpn. J. Appl. Phys. 17, 283-289.

Schiff, L. I., 1968, Quantum Mechanics (McGraw-Hill, New York), 3rd edition, pp. 283-285.

Schipper, P. E., 1981, Chem. Phys. 57, 105-119.

Schuster, P., A. Karpfen, and A. Beyer, 1980, in Molecular Interactions, H. Ratajczak and W. J. Orville-Thomas, Eds. (Wiley, Chichester) pp. 117-149.

Siebrand, W., 1965, J. Chem. Phys. 42, 3951-3954.

Smith, G. C., 1968, Phys. Rev. 166, 839-847.

Sztucki, J. and W. Strek, 1988, Chem. Phys. 124, 177-186.

Thirunamachandran, T., 1979, Proc. Roy. Soc. London A365, 327-343.

Thirunamachandran, T., 1988, Phys. Scripta T21, 123-128.

Tinoco, I., 1975, J. Chem. Phys. 62, 1006-1009.

Tulub, A. V. and K. Patzer, 1968, Phys. Stat. Sol. 26, 693-700.

Van Grondelle, R., 1985, Biochim. Biophys. Acta 811, 147-195.

Van Labeke, D., P. H. Grossel, and J. M. Vigoureux, 1988, J. Chem. Phys. 88, 3211-3215.

Varsanyi, F. and G. H. Dieke, 1961, Phys. Rev. Lett. 7, 442-443.

Vigoureux, J. M., 1983, J. Chem. Phys. 79, 2363-2368.

Vigoureux, J. M., P. Grossel, D. Van Labeke, and C. Girard, 1987, Phys. Rev. A35, 1493-1502.

Von der Linde, D., 1977, in Ultrashort Light Pulses-Picosecond Techniques and Applications,

S. L. Sfrapiro, Ed. (Springer-Verlag, Berlin) pp. 203-273.

Wallace, R., 1966, Mol. Phys. 11, 457-470.

Ward, J. F., 1965, Rev. Mod. Phys. 37, 1-18.

White, J. C., 1981, Opt. Lett. 6, 242-244.

Wiedeman, L., M. E. Fajardo, and V. A. Apkarian, 1987, Chem. Phys. Lett. 134, 55-59.

Wiedeman, L., M. E. Fajardo, and V. A. Apkarian, 1988, J. Phys. Chem. 92, 342-346.

Woolley, R. G., 1971, Proc. Roy. Soc. Lond. A321, 557-572.

Yakovlenko, S. I., 1973, Sov. Phys. JETP 37, 1019-1022.

Yu. Y. C., D. W. Setser, and H. Horiguchi, 1983, J. Phys. Chem. 87, 2199-2209. 